This document is downloaded from DR-NTU (https://dr.ntu.edu.sg) Nanyang Technological University, Singapore.

\title{
All-atom molecular dynamics simulation with levels of polarization
}

Sun, Tiedong

2015

https://hdl.handle.net/10356/62216

https://doi.org/10.32657/10356/62216 

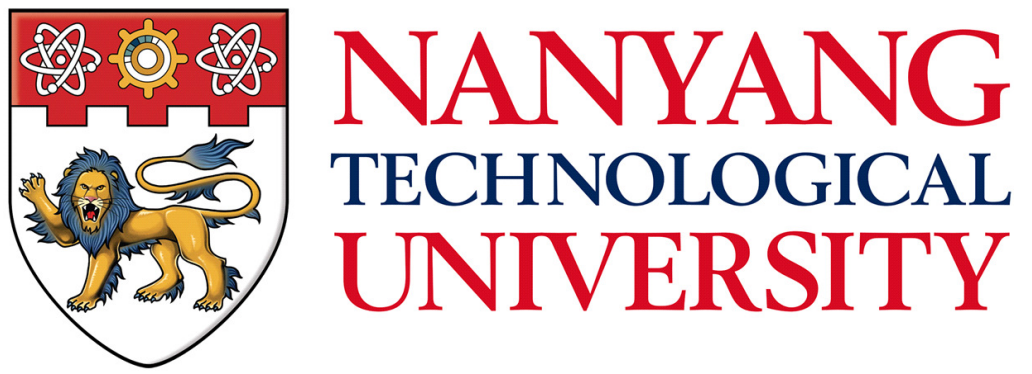

\section{All-atom Molecular Dynamics Simulation With LeVELS OF POLARIZATION}

\section{SUN TIEDONG}

School of Physical and Mathematical Sciences

A thesis submitted to the Nanyang Technological University in partial fulfilment of the requirement for the degree of

Doctor of Philosophy 



\section{ACKNOWLEDGEMENT}

I want to express my sincere gratitude to all people who have helped me during my $\mathrm{PhD}$ study, especially my family, who always support me unconditionally. I'd like to thank my wife and daughter who have brought me much joy and reminded me what it's all about. The gratitude should also be extended to my parents, who have given me everything possible.

I'd like to thank my supervisor, Dr. Zhang Dawei for all the helpful suggestions and guidance. I'm also grateful to Prof. Mei Ye, Dr. Xu Zhijun and Dr. Wei Caiyi. Many issues and obstacles in my PhD study cannot be resolved without their help. My colleagues in our group, Duan Rui, Yang Hong, Cui Jinglan, Yip Yew Mun, especially Raudah Lazim, are very supportive and helpful during my PhD study. They are all easy to work with and greatly appreciated. It's been a great time and beautiful memory working with all these people. Great thanks is also attributed to undergraduate students who have worked with me.

I thank the research scholarship from NTU for the financial support, technicians in the high performance computing center of NTU for their technical support.

Last, but not least, gratitude is expressed to Dr.Ji Changge and Dr.John D. Chodera for their help in coding. It would be impossible to finish my study without helps from them. 


\section{Contents}

\begin{tabular}{ll}
\hline Acknowledgement & 1
\end{tabular}

\begin{tabular}{ll}
\hline Contents & 3 \\
\hline
\end{tabular}

\begin{tabular}{lr}
\hline Abstract & 7
\end{tabular}

\begin{tabular}{lr}
\hline List of Figures & 9
\end{tabular}

\begin{tabular}{ll}
\hline List of Tables & 11
\end{tabular}

\begin{tabular}{ll}
\hline List of Abbreviations & 13
\end{tabular}

$\begin{array}{lll}1 & \text { Basic Theory } & 15\end{array}$

1.1 Introduction . . . . . . . . . . . . . . . . . . . . . . . . 15

1.2 Molecular Dynamics Simulation . . . . . . . . . . . . . . . . . 16

1.3 Force Field . . . . . . . . . . . . . . . . . . . . . . . . . . . . . . . 17

1.4 Electrostatic Polarization in Simulation . . . . . . . . . . . . . . . . 19

$1.5 \quad \mathrm{MFCC}$ and $\mathrm{PPC}$. . . . . . . . . . . . . . . . . . . . . . . . 19

2 Molecular Dynamics Study of Biomolecules Interacting with Graphene 25

2.1 Protein Molecules Interacting with Graphene . . . . . . . . . . . . . 26

$2.1 .1 \quad$ Introduction . . . . . . . . . . . . . . . . . . . . 26

2.1 .2 Simulation Method . . . . . . . . . . . . . . . . . . . 27

2.1 .3 Results and Discussion . . . . . . . . . . . . . . . . 30

2.1 .4 Summary $\ldots \ldots \ldots \ldots$. . . . . . . . . . . . . . 41 
2.2 Short dsDNA Sandwiched Between Graphene Layers . . . . . . . . 42

$2.2 .1 \quad$ Introduction . . . . . . . . . . . . . . . . . . . . . . 42

$2.2 .2 \quad$ Computation Method . . . . . . . . . . . . . . . . . . . 43

2.2 .3 Results and Discussion . . . . . . . . . . . . . . . . . . . 46

2.2 .4 Summary . . . . . . . . . . . . . . . . . . . . . 55

2.3 Concluding Remarks . . . . . . . . . . . . . . . . . . . . . 55

\begin{tabular}{|lll}
\hline 3 & Backbone Fluctuating Charge Model & 57
\end{tabular}

3.1 Introduction . . . . . . . . . . . . . . . . . . . . . . . . . 57

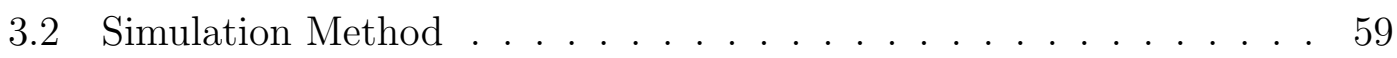

$3.2 .1 \quad$ Polarizable Hydrogen Bond model . . . . . . . . . . . . . . . 59

$3.2 .2 \quad$ Semi-fluctuating Charge model . . . . . . . . . . . . 60

3.2 .3 MD simulation . . . . . . . . . . . . . . . . . . 61

3.2 .4 Trajectory analysis $\ldots \ldots \ldots . \ldots . \ldots . \ldots 63$

3.3 Results . . . . . . . . . . . . . . . . . . . . . . . . . 64

3.3.1 HP7 and chignolin: $\beta$-hairpin . . . . . . . . . . . . . . . 64

$3.3 .2 \quad$ PDCD5-(1-26): $\alpha$-helix . . . . . . . . . . . . 74

3.4 Discussion . . . . . . . . . . . . . . . . . . 79

4 Environment Response Polarized Protein Specific Charge 81

4.1 Introduction . . . . . . . . . . . . . . . . . . . . . . . . . . . . 81

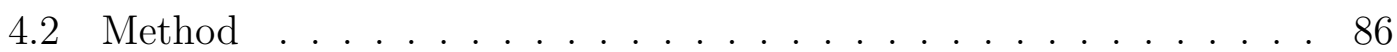

4.2 .1 General Considerations . . . . . . . . . . . . . . . . . . 86

$4.2 .2 \quad$ Selection Of The Updating Criterion $\ldots . . . . . . .87$

4.2 .3 Implementation . . . . . . . . . . . . . . . . . . . . . . . 91

4.2 .4 Molecular Dynamics Protocol . . . . . . . . . . . . . . . . . 92

4.3 Results And Discussion . . . . . . . . . . . . . . . . . . . . . . . . . 94

$4.3 .1 \quad$ Distinguishing Open And Closed States . . . . . . . . . . . . 94

4.3 .2 Dynamics of the WpD loop . . . . . . . . . . . . . . . 96

4.3 .3 The Importance of CYS403 … . . . . . . . . . . . . . . . 99

4.3 .4 Computation Performance . . . . . . . . . . . . . . . . 101

4.4 Conclusion . . . . . . . . . . . . . . . . . . . . . . 102 
5 Concluding Remarks and Future Perspectives 103

\begin{tabular}{ll}
\hline List of Publications & 105
\end{tabular}

\begin{tabular}{ll}
\hline References & 107
\end{tabular} 


\section{ABSTRACT}

Molecular dynamics (MD) simulation has become an indispensable tool in computational chemistry. It is believed that, to produce reliable results, polarization effect must be included in MD simulation. In this thesis, several studies with MD simulation is conducted with different levels of polarization. First, conventional molecular dynamics simulation without polarization is performed to study interactions between graphene and biomolecules. Interesting results are presented, but more fascinating phenomenon and properties cannot be explored with methods at this level. Then protein molecular dynamics simulations are performed with models representing two levels of polarization, namely fluctuating backbone charge and polarized protein-specific charge updating. The results showed that the newly developed polarized protein-specific charge updating scheme, named ERPPC was promising in several perspectives. It incorporates polarization into MD simulation by varying atomic charges periodically. Simulations show that ERPPC reproduced loop dynamics of enzyme YopH. At the same time, it only consumes about 2.5 times computing time of classical molecular dynamics simulation. Further development is expected on ERPPC to improve both accuracy and efficiency. 


\section{List of Figures}

1.1 The MFCC scheme . . . . . . . . . . . . . . . . . . . . . . 20

1.2 Work flow of deriving PPC $\ldots \ldots \ldots \ldots \ldots$

2.1 Simulation setup of sandwiched protein-graphene system $\ldots \ldots .29$

$2.2 \quad$ Averaged structural parameters during protein-grahene simulations. 31

2.3 Time evolution of secondary structure in protein-grahene simulation. 32

$2.4 \quad$ Statistics of van der Waals energy change and solvation free energy change. . . . . . . . . . . . . . . . . . . 34

$2.5 \quad$ Free energy landscape obtained using RMSD and Radius of gyration in protein-grahene simulations. . . . . . . . . . . . . . 36

$2.6 \quad$ The distance between residue COMs and central plain of graphene

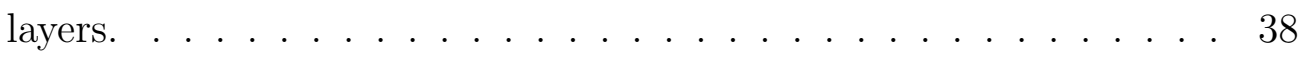

2.7 DNA-graphene simulation setups. . . . . . . . . . . . . . 45

2.8 DNA orientation as a function of time. . . . . . . . . . . . . 48

2.9 Inter-chain hydrogen bond number between ending bases as a function of time. . . . . . . . . . . . . . . . . . . 50

$2.10 \pi$ stacking interactions between DNA and graphene. . . . . . . . 52

2.11 Cross-graphene interactions of DNA. . . . . . . . . . . . 54

3.1 Distribution of all-atom root mean square deviation of $\beta$-hairpins . 66

3.2 Free energy landscape of HP7 at $340 \mathrm{~K}$ and chignolin at $310 \mathrm{~K}$. . . 67

$3.3 \quad$ Fraction of time in native structure in simulations of $\beta$-hairpin . . 68

3.4 Backbone hydrogen bond occupancy and energy of HP7 and chignolin 70 


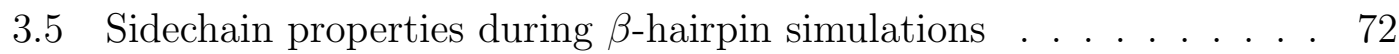

$3.6 \quad \mathrm{~K} 1$ - W10 side chain distance distribution . . . . . . . . . . . . . . 73

3.7 Helical content distribution in helical simulations with all models . $\quad 75$

3.8 Fraction of time in $\alpha$-helical conformation per residue . . . . . . . . 76

3.9 Statistics of $i+4 \rightarrow i$ hydrogen bond occupancy and length . . . . 77

3.10 Nonnative backbone hydrogen bond occupancy. . . . . . . . . . . . 78

4.1 Potential RMS per residue . . . . . . . . . . . . . . . . . . . 89

4.2 Distribution of potential RMS fitted to Gaussian distribution . . . . 90

4.3 Overlap of open and closed backbone structures of YopH determined with PCA . . . . . . . . . . . . . . . . . . . . 95

4.4 Backbone B-factors of YopH per residue . . . . . . . . . . . . . . . 97

4.5 C $\alpha 357-\mathrm{C} \alpha 407$ distance as a function of time . . . . . . . . . . . . . 98

$4.6 \mathrm{C} \alpha 357-\mathrm{C} \alpha 407$ distance as a function of time in simulations with protonated C403 . . . . . . . . . . . . . . . . 100 


\section{List of Tables}

$2.1 \quad$ Aromatic ring interactions with graphene. Percentage of time where $\theta<20^{\circ} \ldots \ldots \ldots \ldots \ldots$. . . . . . . . . . . . . . . . . . . . .

3.1 Summary of MD simulations carried out to test PHB and SFC models 62

4.1 Computing time consumption of AMBER and ERPPC simulations 101 


\section{List of Abbreviation}

$\begin{array}{ll}\text { AIMD } & \text { Ab Initio Molecular Dynamics } \\ \text { CG } & \text { Coarse Graining } \\ \text { CGMD } & \text { Coarse-Grain Molecular Dynamics } \\ \text { CPU } & \text { Central Processing Unit } \\ \text { Hbond } & \text { Hydrogen Bond } \\ \text { MD } & \text { Molecular Dynamics } \\ \text { MFCC } & \text { Molecular Fragmentation with Conjugated Caps } \\ \text { MM } & \text { Molecular Mechanics } \\ \text { PB } & \text { PoissonBoltzmann } \\ \text { PPC } & \text { Polarized Protein-specific Charge } \\ \text { QC } & \text { Quantum Chemistry } \\ \text { QM } & \text { Quantum Mechanics } \\ \text { REMD } & \text { Replica Exchange Molecular Dynamics } \\ \text { RESP } & \text { Restrained Electrostatic Potential } \\ \text { SMD } & \text { Steered Molecular Dynamics }\end{array}$





\section{Chapter 1}

\section{Basic Theory}

\subsection{Introduction}

Understanding the past and future of physical system is depending on time evolution relationship described in physical theories, also known as equations of motion. To derive time evolution of physical system, i.e. dynamics, one is often required to calculate coordinate integration over time, which is usually beyond the calculation capability of human being, especially with systems with high degrees of freedom. In modern days, the advance in computing provides the capability of studying time evolution of physical system of decent size. The time integration algorithm, which is usually iteration, can be implemented and run as long as we need. In condensed phase physics, biological researches and material science, these methods and techniques are extensively applied to study all sorts of questions, ranging from protein-ligand binding to adsorption on material surface.

Since the introduction of computer to dynamics study, molecular modeling and dynamics has become more and more essential in modern science, as it has grown into a significant branch of computer modeling with all its developments ${ }^{1}\left[\frac{6}{6}\right.$ in basic theory as well as practical techniques. In this chapter, a brief overview of several key aspects of molecular modeling and dynamics will be given, in order to depict the background that works in this thesis are based on. It is not intended to be an extensive review of molecular dynamics. Only the founding theory of molecular 
dynamics shall be presented. And the logical reasoning for works in this thesis should emerge.

\subsection{Molecular Dynamics Simulation}

Generally speaking, molecular dynamics simulation is the computing technique and a set of methods that are used to study time-dependent as well as statistical properties of physical system, at atom level. It can be categorized according to scale, i.e. ab initio molecular dynamics (AIMD), ${ }^{7-10}$ classical molecular dynamics $(\mathrm{MD})^{11112}$ and coarse-grained molecular dynamics (CGMD) ${ }^{3 / 4}$ There are also techniques that are designed to improve sampling efficiency, such as replica exchange molecular dynamics (REMD) $13[14 \text { and steered molecular dynamics (SMD) })^{15}$ etc.. To study real life question with computer, a suitable model is required to represent real systems. In molecular dynamics simulation, the model consists of two parts, a set of variable that evolves with time and an energy function that decides how these variable evolves with time, i.e. coordinates and potential.

In classical molecular dynamics, the system is usually represented by atoms connected with harmonic bonds. The coordinates $\vec{x}$ are the variables that evolves over time. The motion of atom $i$ is regulated by Newton's equation of motion.

$$
-\frac{\partial U(\vec{x})}{\partial \vec{x}_{i}}=m_{i} \ddot{\vec{x}}_{i}
$$

where $U(\vec{x})$ is the potential function regulating the system.

With the equation of motion, integration algorithms are developed to conduct time integration with the power of computers. The most simple way of solving a second order differential equation, such as Eqn 1.1, is to propagate from boundary which is initial coordinates in this case. Verlet integration, $\frac{16}{16}$ also known as leapfrog method, which is a commonly used integration algorithm in molecular dynamics was developed in this way initially. First, calculate one step forward in time

$$
\vec{x}_{1}=\vec{x}_{0}+\dot{\vec{x}}_{0} \Delta t+\frac{1}{2} \ddot{\vec{x}}_{0} \Delta t^{2}
$$


where $\vec{x}_{0}$ and $\dot{\vec{x}}_{0}$ are needed to initiate the simulation. Then iterate for step number $\mathrm{n} \rightarrow \infty$ with

$$
\vec{x}_{n+1}=2 \vec{x}_{n}-\vec{x}_{n-1}+\ddot{\vec{x}}_{n} \Delta t^{2}+O \Delta t^{4}
$$

Note the error of this algorithm is of the order of $\Delta t^{4}$. Also from Eqn 1.3 , we can see no velocity variable is involved in the time integration. However, many physical quantities, such as pressure, is relying on velocity information from time integration. The velocities in Verlet algorithm are calculated as following.

$$
\vec{v}(t)=\frac{\vec{x}(t+\Delta t)+\vec{x}(t-\Delta t)}{2 \Delta t}+O\left(\Delta t^{2}\right)
$$

A more popular algorithm to integrate is the Velocity Verlet method. ${ }^{17}$ It uses the following relationship to evolve over time.

$$
\begin{aligned}
& \vec{x}(t+\Delta t)=\vec{x}(t)+\vec{v}(t) \Delta t+\frac{1}{2} \vec{a}(t) \Delta t^{2} \\
& \vec{v}(t+\Delta t)=\vec{v}(t)+\frac{\vec{a}(t)+\vec{a}(t+\Delta t)}{2} \Delta t
\end{aligned}
$$

where $\vec{v}$ and $\vec{a}$ are velocity and acceleration respectively. The velocities are calculated explicitly as the integration goes in Velocity Verlet. It can be shown that the error in Velocity Verlet is of the same order as the Verlet algorithm. Velocity Verlet is preferred as velocities and coordinates are calculated at the same time points.

\subsection{Force Field}

The potential function $U(\vec{x})$ plays a pivotal role in time integration of molecular systems, as can be seen in previous section. All interactions are described by the potential function. It is up to the researcher to choose suitable potential function to study the system of interest. In ab initio molecular dynamics, $U(\vec{x})$ is determined with electronic structure theories, which usually is quite accurate and requires much more CPU time. In classical MD and coarse-grained MD, the potential 
function is empirical with parameters to be derived. Some of these parameters are derived from experimental measurements, some from ab initio calculation results. Empirical potentials are often of simple form and easier to compute.

The empirical potential function adopted by atomic MD is often called "force field", as it produces "force" in MD simulation. In classical molecular dynamics, molecules are represented by atoms and bonds. A typical energy expression from classical force field is divided into two parts, bonded and nonbonded energies.

$$
\begin{aligned}
E_{\text {total }} & =E_{\text {bonded }}+E_{\text {nonbonded }} \\
E_{\text {bonded }} & =E_{\text {bond }}+E_{\text {angle }}+E_{\text {dihedral }} \\
E_{\text {nonbonded }} & =E_{\text {electrostatic }}+E_{\text {vanderWaals }}
\end{aligned}
$$

Each energy term is given a specific form and a number of parameters. The parameters are determined with experimental observations or higher level calculations. For instance, the AMBER force field ${ }^{18119}$ has the following form of potential.

$$
\begin{aligned}
E_{\text {total }}= & \sum_{\text {bonds }} K_{r}\left(r-r_{e q}\right)^{2}+\sum_{\text {angles }} K_{\theta}\left(\theta-\theta_{e q}\right)^{2}+\sum_{\text {dihedrals }} \frac{V_{n}}{2}[1+\cos (n \phi-\gamma)]+ \\
& \sum_{i<j}\left[\frac{A_{i j}}{R_{i j}^{12}}-\frac{B_{i j}}{R_{i j}^{6}}\right]+\sum_{i<j} \frac{q_{i} q_{j}}{\epsilon R_{i j}}
\end{aligned}
$$

The first three terms are bonded energy terms. The last two are van der Waals and electrostatic energy terms respectively. Other second generation force fields have similar potential functions which may vary a little from this function form. $20+22$

Force field has achieved great success in simulations with biological molecules, varied chemical systems and structures and material sciences. Recently, great effort has been put on developing more accurate force field in molecular modeling community. The performance of force fields is advanced gradually as new models and new parameters ${ }^{23 \mid 24}$ are proposed and implemented. 


\subsection{Electrostatic Polarization in Simulation}

In classical molecular dynamics, electrostatic interaction is simply modeled as fixed point charge interacting by Coulomb's law $\frac{\sqrt{1820}+22}{22}$ as shown in equation 1.10 . The electrostatic energy of modeled system is just the sum of electrostatic interaction energy of all point charge pairs. This description is overly simple in the eyes of modern computational chemists. Though polarization energy can be partially accounted for by carefully chosing valus for atomic charge, i.e. so-called "effective energy", polarization effect, which comprise a significant part in electrostatic interactions, $\stackrel{25}{25}$ is ill-represented in classical force field.

It is a consensus among the molecular modeling community that including polarization effect into molecular dynamics simulation is both necessary and challenging. There are evidences ${ }^{26 \mid 27}$ that inclusion of polarization effect could produce experimental phenomenon that classical force field fails to reproduce. Many research groups have studied polarization effect and proposed a number of models $^{28} \sqrt{32}$ and techniques to incorporate polarization effect into molecular dynamics. These models are developed from all kinds of perspective. For example, polarizable force field takes polarization effect into account by introduce extra degree of freedom. Fluctuating charge models ${ }^{32} \sqrt[35]{35}$ allow atomic partial charge varying during MD simulation, that is, considering atomic charges as variables. Other

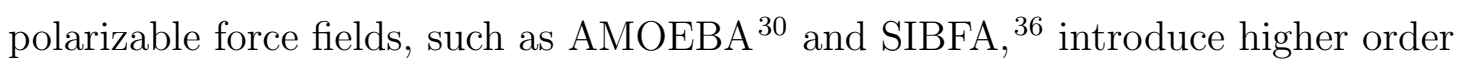
electrostatic multipoles to describe polarization explicitly. Normally, polarizable force fields consume several times CPU time ${ }^{\sqrt{30 \mid 37}}$ than conventional molecular dynamics simulation. Greater development on polarizable force field is expected as computing capability grows.

\subsection{MFCC and PPC}

Computational chemists are always tempted to perform quantum mechanical calculations on larger and larger molecules or molecular clusters. However, it is not always possible even with the fastest computer existing, as quantum mechanical 
(a)

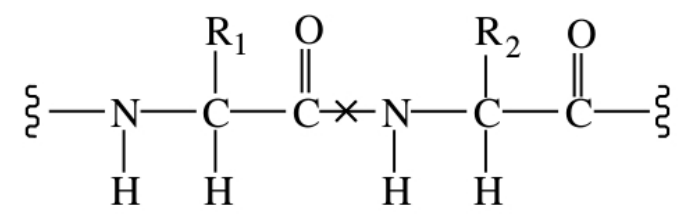

(b)

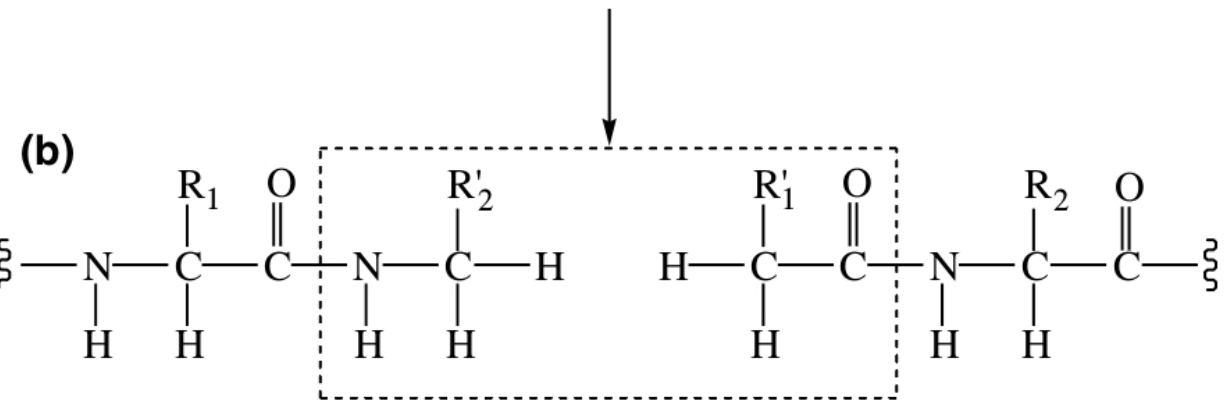

(c)<smiles>[R12]CNC(=O)C([R1])[2H]</smiles>

Figure 1.1: The MFCC scheme: (a) cutting the peptide bond (b) a pair of conjugated caps are inserted (c) the conjugate caps are join to form "concap". This figure is from Gao et.al.. ${ }^{44}$

algorithms scale exponentially with number of degrees of freedom ${ }^{38}$ that is being considered. New algorithms and techniques are to be found with linear scaling. ${ }^{39140}$ Molecular fragmentation with conjugated caps $(\mathrm{MFCC})^{41142}$ is one of such linear scaling methods to perform quantum calculation on large molecules, such as protein. It is one of a group of similar fragmentation methods ${ }^{43}$ that achieve better scaling performance. The basic procedure of these methods is to divide large system into small subsystems. Performing calculation on subsystems can significantly reduce computation cost. Computation methods using fragmentation in quantum mechanical calculation of large system is reviewed by Gordon et.al.. ${ }^{43}$

MFCC is proposed by Zhang et.al. $\stackrel{41422}{ }$ first. The basic idea of MFCC is shown in figure 1.1$]^{44}$ In MFCC calculation, molecule A-B, usually peptide or protein, is cut into smaller fragments $\mathrm{A}$ - and $-\mathrm{B}$, e.g. residues. Caps are inserted to both fragments at the cutting point to form two new capped molecules A-c and $\mathrm{c}^{*}$-B. 
Quantum mechanical calculation is conducted on newly formed molecules, as well as the "concap" $\mathrm{c}^{*}$-c. Then to recover the electronic structure and properties of the original molecule $\mathrm{AB}$, simple addition and subtraction is applied to all fragments. For electron density, we have ${ }^{44}$

$$
\begin{aligned}
& \rho=\sum_{i=1}^{N} \rho_{i}-\sum_{i=1}^{N-1} \rho_{i}^{c c} \\
& \mu=\sum_{i=1}^{N} \mu_{i}-\sum_{i=1}^{N-1} \mu_{i}^{c c}
\end{aligned}
$$

where $\rho_{i}$ and $\mu_{i}$ are electron density and of the $i$ th fragment with caps and electrostatic potential resulted from the $i$ th fragment, respectively. And $\rho_{i}^{c c}$ and $\mu_{i}^{c c}$ are electron density and electrostatic potential from the concap. Here, the conjugated caps preserve the local environment around the cutting point. With the assumption of electron localization, MFCC allows recovering properties of the whole molecule with simple addition and subtraction. It has been proved the error arise from fragmentation is acceptable in studies of proteins and other biological systems. $.41|42| 44,46$

Improvement on classical force field has been attempted with the help of MFCC. The atomic charge in classical force field is residue specific, $\frac{18 \mid 19}{1}$ that is, the same set of charge is used for a specific residue species in MD simulations no matter the environment that residue experiences. In reality, two residues of the same kind in two protein molecules, or even in the same protein but different positions, have different electronic structure, hence different charge distribution. We ought to utilize charge that corresponds to the charge distribution under its current environment, so that the electrostatics is accurate. The residue charge under local environment can be obtained by conducting MFCC calculation as electrostatic potential can be calculated easily as in equation 1.12 . This idea is compatible with AMBER force field, since the atomic charge of AMBER force field is derived in a similar way. $\frac{18 \mid 19}{10}$ The newly generated charge is no longer residue-specific. Each protein with a specific structure will have a unique set of atomic charge. On the 
other hand, the polarization effect is included as inter residue interaction that has been accounted for in MFCC calculation. Therefore, we have the name, polarized protein-specific charge (PPC) $\stackrel{[47}{7}^{T}$ The common work flow of deriving PPC is shown in figure 1.2 , 47

As can be seen from the idea and implementation of PPC, it depends on the structure that was fed to the deriving process. The structure dependency can cause inaccuracy sometimes. A more robust fitting method is proposed by Zeng

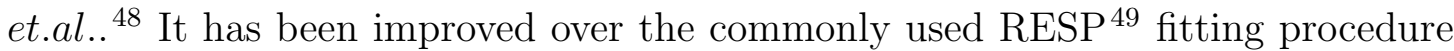
and eliminated some structure dependency of PPC while preserving its proteinspecific feature. 


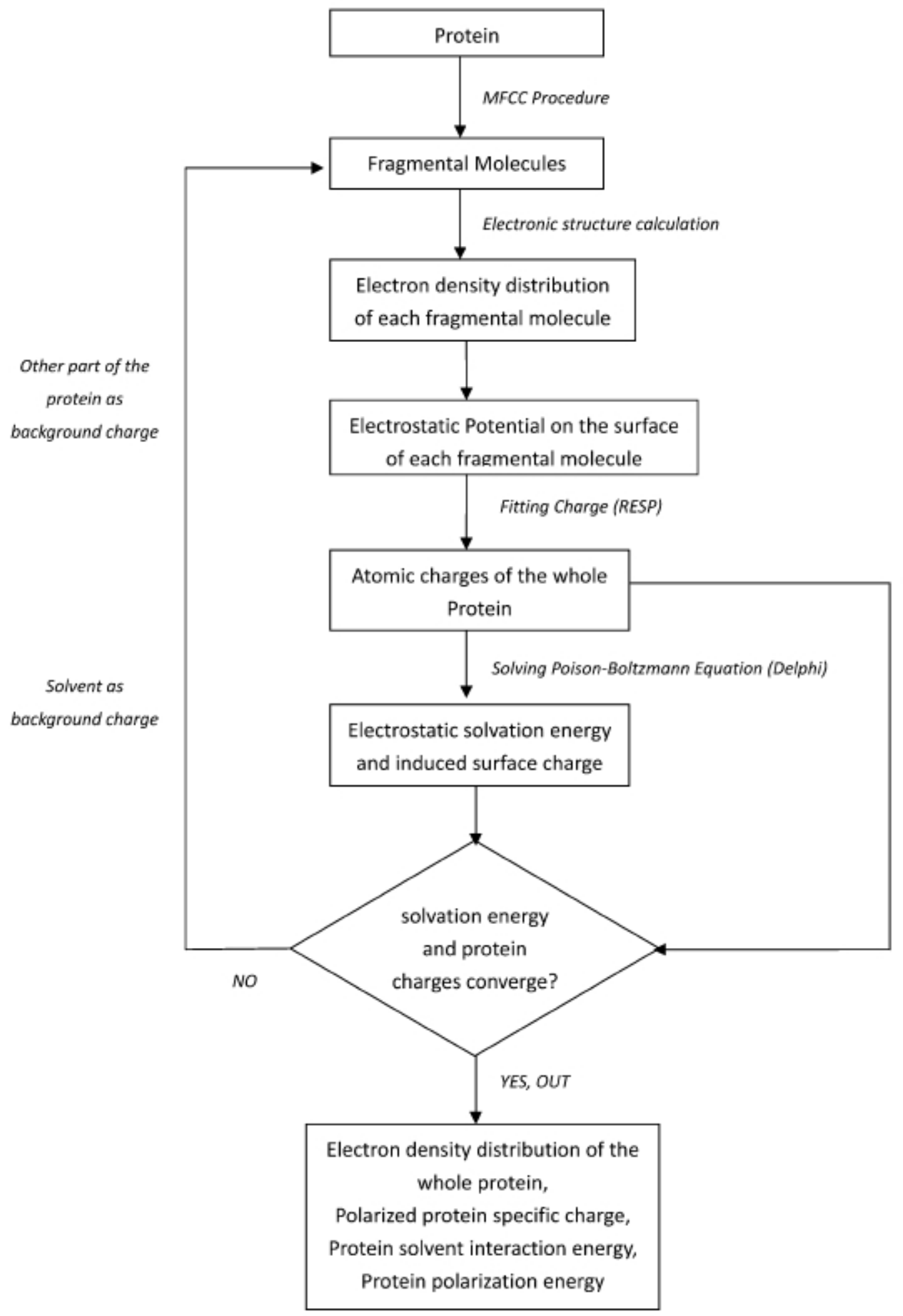

Figure 1.2: Work flow of deriving PPC with MFCC-PB. Figure is directly from Ref 47 


\section{Chapter 2}

\section{Molecular Dynamics Study of Biomolecules Interacting with Graphene}

Graphene biofunctionalization has been rapidly developed in recent years. ${ }^{50151}$ Due to its unique property and vast application possibilities, grephene opens up a new world of interacting and manipulating biologically interesting entities, from biomacromolecules to cell, at nanometer scale. The attraction of graphene to scientific society originates from graphene's structural and electronic uniqueness. Single layer graphene is a one atom thick planar surface with superlative mechanical strength $^{52}$ and great thermal conductivity. ${ }^{53}$ Other known materials are not comparable to graphene in these perspectives. Electronically, the honeycomb structure of graphene could hold a large amount of conjugated $\pi$-electrons. Many unique properties such as parabolic energy dispersion ${ }^{54}$ and quantum Hall effect ${ }^{[55}$ at room temperature are due to the electronic features of graphene. The possibilities also rise from all derivatives of graphene, 0-dimensional fullerene, 1-dimensional carbon nanotubes etc., and numerous ways of modifying these species to suit our needs. To name a few, functionalized graphene oxide (GO) is produced to deliver hydrophilic cancer drugs. ${ }^{56}$ Single-strand DNA (ssDNA) is tethered to graphene surface to make it possible to build a DNA detector. $\frac{57}{}$ And last, enzymes, such as lysozyme, are adsorbed on the surface of graphene or graphene oxide to achieve specific functions. $\frac{58}{58}$ 
Chapter 2. Molecular Dynamics Study of Biomolecules Interacting with GRAPHENE

In this chapter, studies of protein and DNA duplex interacting with pristine graphene are presented. It should be noted that the study of biofunctionalization of grephene and its derivatives is still in its infancy. The studies here are focused on describing graphene with minimalist model and producing reasonable results. Hence, a relatively simple force field model shall be adequate to achieve our goal.

\subsection{Protein Molecules Interacting with Graphene}

\subsubsection{Introduction}

Graphene and graphene oxide can be functionalized by protein through either physical adsorption ${ }^{58}$ or chemical bonding. ${ }^{[59}$ For example, bovine serum albumin (BSA) has been exploited to tune the solubility of graphene sheets. ${ }^{60}$ Based on electrostatic adherence, both positive and negative charged nanoparticles can be decorated on the BSA-graphene interface at suitable $\mathrm{pH}$ and temperature. Another example is that horseradish peroxidase (HRP) and lysozyme can be immobilized on graphene oxide spontaneously,$\sqrt[58]{ }$ indicating the oxygen enriched graphene oxide might be a good agent for protein adsorption so as to realize various functions.

In spite of all the effort that was made to create novel protein-graphene complex, the molecular detail and mechanism of protein-graphene interaction is still unclear. It is urgent to complete the theory of protein-graphene interaction, so that it can be used to provide guidance to design and produce these complexes with great use. To acquire more information of graphene-protein interaction, all-atom molecular dynamics (MD) simulation is the first candidate approach to adopt. The simple model and statistical interpretation of MD simulation shall give an good overall understanding on protein-graphene interaction.

In this study, we are trying to shed light on the mechanism of protein-graphene interaction by means of molecular dynamics simulations. We carried out studies on the direct interactions between graphene and two model protein molecules in a series of simulations. These two protein molecules have different structural features, i.e. one is helix bundle and the other is $\alpha \beta$ hybrid. The results showed 
Chapter 2. Molecular Dynamics Study of Biomolecules Interacting with GRAPHENE

that the van der Waals attraction from graphene layer was strong enough to denature protein molecules packed by hydrophobic interactions. Together with the hydrophobic interaction introduced by its surface, graphene can alter protein structure significantly. However, some tightly packed protein or protein subunits can survive from the denaturing effect of graphene. These results suggest that the van der Waals interaction between graphene and protein molecules plays a significant part in forming protein-graphene complexes, which should be considered while trying to functionalize graphene. The protein molecule that is chosen to adsorb to graphene surface must be strong enough, otherwise it is very likely to have a complex without any bioactivity in the end.

\subsubsection{Simulation Method}

To examine the direct impact of graphene on protein molecules, we chose two structurally dissimilar proteins, GA module from protein PAB ${ }^{61}$ (PDB ID 1PRB) and an immunoglobulin binding domain of protein $G^{62}$ (PDB ID 2GB1), as our subjects. GA module is found in a family of surface proteins from different bacterial species. It comprises a left-handed three-helix-bundle. ${ }^{61}$ The structure of protein $\mathrm{G}$ is composed of a four-stranded beta sheet with a helix on top $\frac{62}{6}$ The central two strands ( $\beta$-sheet 1 and $\beta$-sheet 4 ), comprising the NH2- and COOH-termini, are parallel. The outer two strands ( $\beta$-sheet 2 and $\beta$-sheet 3 ) are connected by the helix (see Figure 2.1).

Molecular dynamics simulations were performed on these two protein molecules. Protein molecules, modeled by AMBER99SB ${ }^{63}$ force field, were put inside the space formed by two graphene single layers, to form a sandwich-like configuration. Graphene layers were modeled as Lennard-Jones particles (generic atom type CA in AMBER99SB force field) connected by harmonic bonds. In order to eliminate edge effects, graphene layers were modeled as an infinite plane by creating cross-boundary bonds between graphene molecule and its own periodic image. The graphene layers were parallel to XY-plane of a triclinic box adopting periodic boundary condition. Totally four separations between graphene layers were used 
Chapter 2. Molecular Dynamics Study of Biomolecules Interacting with GRAPHENE

to study the effect of the space size formed by graphene layers on protein stability. Separations in the starting structures are $2.5 \mathrm{~nm}, 3.0 \mathrm{~nm}, 4.5 \mathrm{~nm}$ and $6.0 \mathrm{~nm}$ for GA module, and $3.0 \mathrm{~nm}, 4.0 \mathrm{~nm}, 6.0 \mathrm{~nm}$ and $8.0 \mathrm{~nm}$ for protein G. In Z direction, the two graphene layers were symmetrical to the center of the box and at least 1 nm away from the box edges. One other system without graphene layers was also constructed for each protein (i.e., protein molecule in bulky water simulations). TIP3P waters, ${ }^{64}$ as well as neutralizing ions, were added into the box. All simulations were started from NMR structures of protein molecules, whose centers of mass were located at the center of the box. System setup of the starting point is depicted in Figure 2.1.

All molecular dynamics simulations follow a same procedure. First of all, the potential energy of the whole system is minimized to a point where the maximum force in the system is less than $100.0 \mathrm{~kJ} / \mathrm{mol} / \mathrm{nm}$. Then, a 100 ps constant volume simulation at $300 \mathrm{~K}(\mathrm{NVT})$ was carried out with the position of heavy atoms in graphene and protein molecules held restrained. Following the NVT phase was a 2 ns constant pressure equilibration phase at $300 \mathrm{~K}$ (NPT) with only the heavy atoms in protein molecules held restrained. The last step is performing a $100 \mathrm{~ns}$ production molecular dynamics simulation with no restraint in NPT ensemble. A 2 femtoseconds timestep was realized by constraining all bonds containing hydrogen atoms with LINCS algorithm. ${ }^{67}$ For each setup of each protein, four $100 \mathrm{~ns}$ long trajectories with different initial velocity were obtained, resulting in a total simulation time length of $2.0 \mu \mathrm{s}$ for each protein. All simulations were performed at $300 \mathrm{~K}$ with velocity rescaling thermostat, 68 and at 1 Bar with Parrinello-Rahman barostat, $\frac{69170}{}$ which was coupled only to $Z$ direction in systems with graphene. Pressure in systems without graphene layers was coupled to all three dimensions. Particle-Mesh Ewald (PME) ${ }^{7172}$ method was used to sum the electrostatic interactions. van der Waals interaction was calculated with a cut-off of $1.2 \mathrm{~nm}$. All simulations were performed with GROMACS simulation package.73 


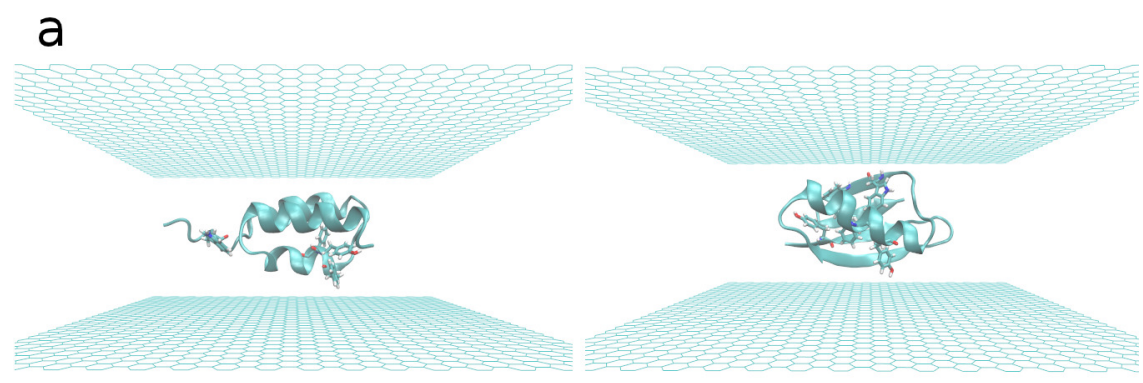

b

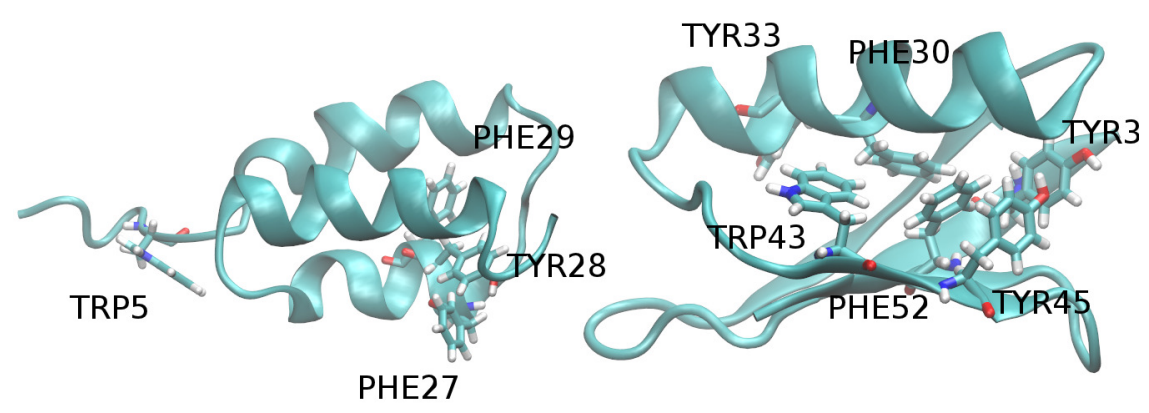

Figure 2.1: Simulation setup of sandwiched protein-graphene system. Protein molecules are placed in between graphene layers. GA module (left) and protein $\mathrm{G}$ (right) are studied here. In the lower figure, all aromatic residues are drawn explicity and labeled with their name and residue number. $\mathrm{VMD}^{65666}$ is used to render this figure. 
Chapter 2. Molecular Dynamics Study of Biomolecules Interacting with GRAPHENE

\subsubsection{Results and Discussion}

\section{Structural changes of protein molecules}

We conducted a series of MD simulations to investigate the mechanism of proteingraphene interaction. Instead of studying interactions between protein molecules and graphene single layer or multiple layers, we built the model system by inserting protein molecules into the space formed by two parallel graphene layers (Figure 2.13). The reason of this setup is that, in real cellular environment, proteins function in a crowded environment. Numerous experiments and simulations $\frac{74}{77}$ showed that the crowding, or confining, effect could affect protein structures significantly. We believe this planar confinement could mimic the confining effect for proteins near the surface of graphenes to some extent. When the separation of graphene bilayer goes beyond $6 \mathrm{~nm}$ for GA module and $8 \mathrm{~nm}$ for protein $\mathrm{G}$, it is very similar with the protein adsorbed onto single graphene layer. For each of our two subjects, GA module and protein $\mathrm{G}$, there were four separations between graphene single layers applied. Four trajectories with the same starting structure, but different initial velocities were obtained for each setup of each protein molecule. In all of our simulation trajectories, protein molecule underwent some conformational changes and reached meta-stable structure within 50 ns.

First, the statistics of root mean square deviations (RMSD) of $\alpha$-carbon atoms and radius of gyration $(\mathrm{Rg})$ of protein molecules are shown in Figure 2.2 (a and b). The data are from the second half (50 ns) of each trajectory. It is clear that inside graphene layers, GA module underwent much more significant conformational change with greater magnitude and variations in both RMSD and Rg values than protein $\mathrm{G}$. The $\alpha$-carbon RMSD values of GA module are higher than $0.4 \mathrm{~nm}$ in most trajectory snapshots of low graphene separation systems (separation 1 to 3 ), which indicates that GA module underwent serious conformational changes and denaturation in these simulations. In large graphene separation system, as well as in bulky water, the average $\alpha$-carbon RMSD value is about $0.3 \mathrm{~nm}$. Besides, there is an obvious trend, which is, the smaller the separation is, the larger the 
a

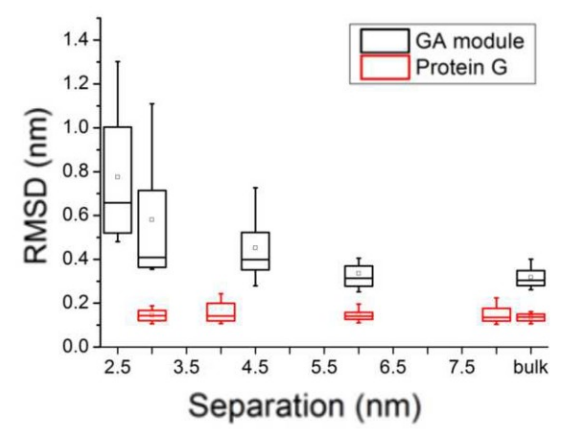

b

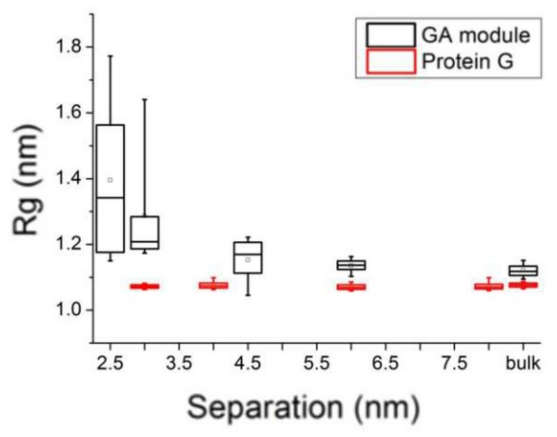

C

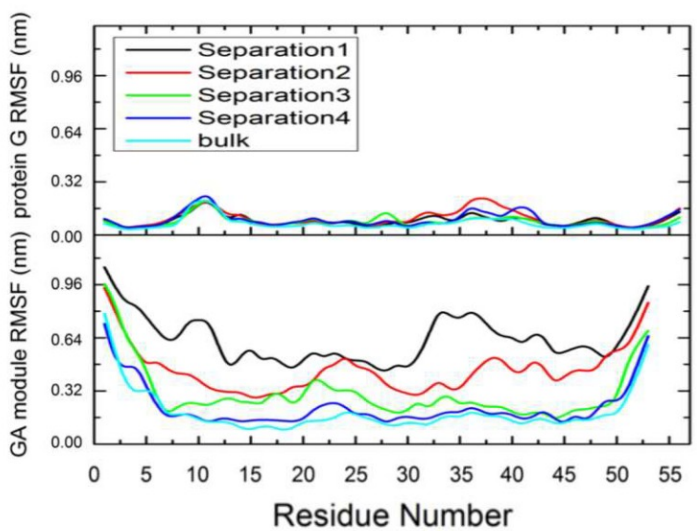

Figure 2.2: Averaged structral parameters during protein-graphene simulations. a: root mean square deviation of $\mathrm{C} \alpha$ statistics. b: radius of gyration statistics. c: root mean square fluctuation (atomic fluctuation) of $\alpha$-cabon atoms as a function of residue number. In the box charts, horizontal boundaries and whiskers denote $10 \%$, $25 \%, 50 \%, 75 \%$ and $90 \%$ of the distribution. The little squares inside box chart indicate mean values. All data are derived from the last $50 \mathrm{~ns}$ of all trajectories. 

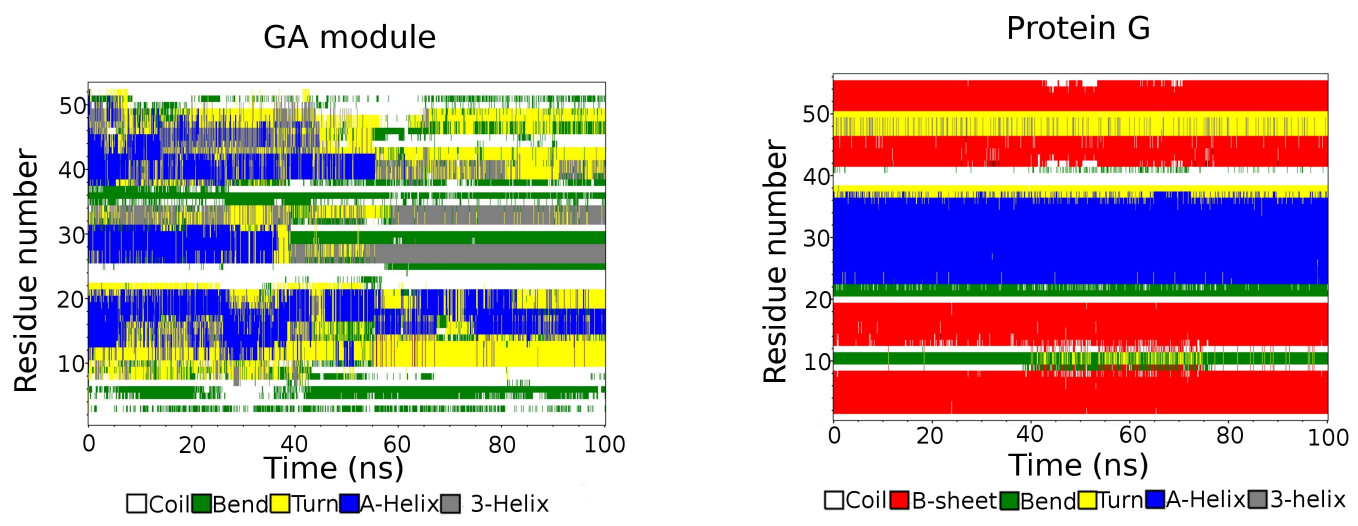

Figure 2.3: Time evolution of secondary structure in protein-graphene simulation. The secondary structure is assigned based on DSSP algorithm ${ }^{78}$ for GA module (left) and protein G (right).

RMSD values and their variation. This indicates that smaller separation could cause greater denaturation of GA module. The same trend exists in the statistical results of radius of gyration. On the contrary, protein $\mathrm{G}$ molecule maintained its NMR structure through all of our simulations. The average RMSD values under four separations are all less than $0.2 \mathrm{~nm}$. The variations of both RMSD value and the $\mathrm{Rg}$ in different separations are much smaller compared to similar situations of GA module. The same observations occur in the root-mean-square fluctuations (RMSF) analysis (shown in Figure 2.2 c) in which the position deviations of each residue were examined to reflect the stability of the protein molecules during the simulation.

To further evaluate the different denaturation of the two dissimilar proteins, the time-evolution of secondary structures $\frac{78}{78}$ of the proteins was also shown in Figure 2.3. As we can see, the three helices (colored blue) of GA module lost most of their structures and turned into structurally loose segments such as random coil or turn. The secondary structure of protein G, however, was preserved very well throughout the simulation.

A folded protein resides in a minimum of its potential energy surface until it is perturbed by denaturing factors, such as heat or interactions with other molecules. 
Chapter 2. Molecular Dynamics Study of Biomolecules Interacting with GRAPHENE

In our case, the denaturing factor is the interaction between protein molecules and graphene layers. From the observation that GA module was denatured while protein G kept its NMR structure in all situations, we can postulate that protein G molecule may reside in a much deeper potential energy well and need to cross a much higher energy barrier to denature than GA module in their folded state. The interaction with graphene layers can induce GA module jumping out of its energy minimum and exploring much wider conformational space. However, it is not strong enough to produce similar effects on protein $G$ molecule. Clearly, the structure of protein as part of the protein-graphene complex is dependent on intrinsic features of protein molecules. Therefore, more investigation on the interactions between graphene layers and protein molecules is needed to help us understand the different effects induced by graphene layers.

\section{Energetics}

Energetically, non-bonded interactions and entropy contribution is responsible for protein conformational change in between graphene layers. Since we adopt an uncharged model of graphene layer, the non-bonded interaction between graphene layers and protein molecule only consists of van der Waals interaction, e.g. LennardJones term of the force field. Therefore, in this study we analysed the LennardJones interaction between graphene layers and protein molecules and plotted the van der Waals energy statistics during simulations in Figure 2.4

In all of our simulations, the van der Waals attraction energy (indicated by negative values) between protein and graphene became stronger as the simulation goes on. However, in the meantime, water molecules are repelled from graphene as well as protein molecules. In other words, part of the water shell around graphene and protein is broken, which causes van der Waals energy rising. We divide our system into three groups: protein, graphene, and other molecules (water and counter ion). Then van der Waals interaction energies between each pair of these groups are calculated. At last, the sum of all three van der Waals energy changes is taken to account for the van der Waals contribution in the denaturing process. The 

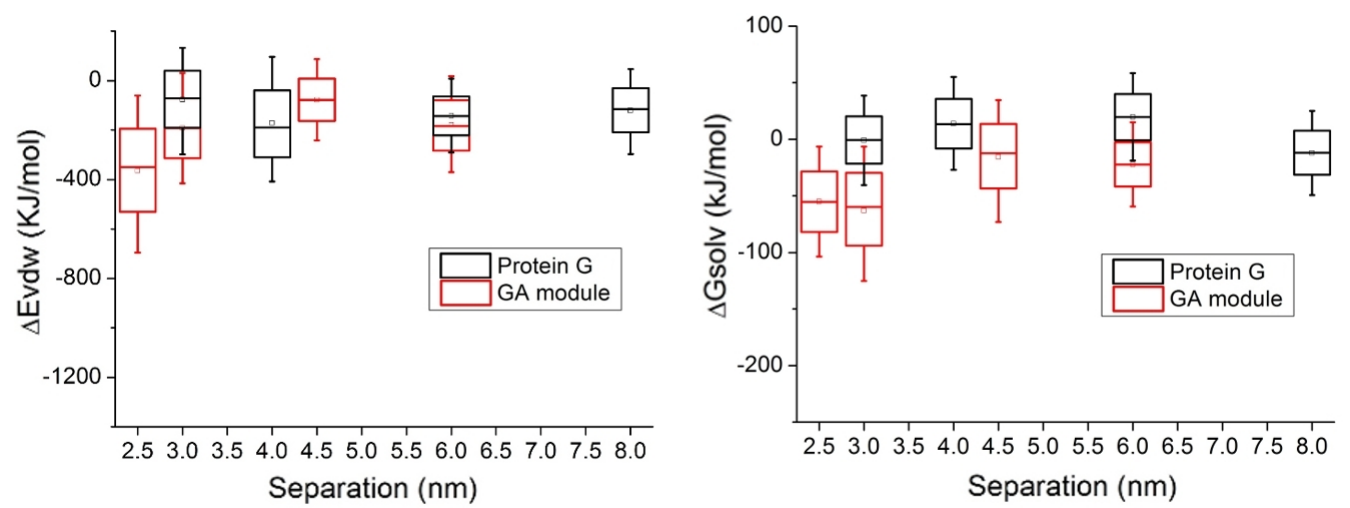

Figure 2.4: Statistics of van der Waals energy change (left) and solvation free energy change (right) during simulations. Data are obtained with the last $50 \mathrm{~ns}$ of all trajectories.

statistics of these sum values $\left(\Delta E_{v d w}\right)$ are plotted in figure 2.4. Again, we see bigger contribution in smaller separation. For protein G, the average van der Waals energy change, $\Delta E_{v d w}$, is about $-150 \mathrm{~kJ} / \mathrm{mol}$ in all cases of separations. However, $\Delta E_{v d w}$ values of GA module are more negative than those of protein $\mathrm{G}$. In small separation cases (separation 1 and 2), $\Delta E_{v d w}$ values of GA module could go as low as $-360 \mathrm{~kJ} / \mathrm{mol}$ on average. The big difference in $\Delta E_{v d w}$ can be attributed to the conformational change. As a result of protein denaturing, protein molecule becomes more flexible than in its native states. This flexibility allows protein molecule to extend itself to accomplish stronger interaction with graphene layers. Generally, flexible molecules can easily get into a position where their centers of mass (COM) are nearer to graphene when they approach graphene layers. In our case, GA module went through more significant conformational changes which made it more flexible on the surface of graphene layer.

Another important factor which may affect protein behaviour significantly is solvation. Here, we adopted a simple and efficient method, termed weighted solvent accessible surface area (WSAS), ${ }^{79}$ to analyze the solvation energy change during the conformational changes of protein molecules. In this method, a coefficient obtained by fitting known solvation free energy of hundreds of compounds is 
Chapter 2. Molecular Dynamics Study of Biomolecules Interacting with GRAPHENE

assigned to each type of atoms. Then the solvation energy can be calculated by multiplying the solvent accessible area of each atom of the solute with the assigned coefficient and then adding them together. There is no explicit entropic term in the equation as the entropic contribution to the solvation energy is taken into account implicitly through the weighting coefficients. According to Wang et.al., ${ }^{79}$ the results from WSAS method have a high correlation to those calculated with PBSA, a widely used solvation energy calculation method.

The solvation free energy changes $\left(\Delta G_{\text {solv }}\right)$ of graphene-protein system were plotted in box chart in Figure 2.4. As can be seen, GA module displayed a decrease of $G_{\text {solv }}$ in all simulations. This can be explained by the decrease of solvent accessible surface area of graphene. The denaturation of GA module provides extra flexibility to the protein molecule, which allows GA module occupy more surface area of graphene. This can explain the lower solvation free energy change of GA module comparing to protein G.

It is noted that, the contribution from solvation to protein denaturing is less than that of the van der Waals interaction. The contribution of van der Waals interaction is about 4 to 5 times larger than that of solvation energy. Previously, the toxicity of graphene was more related to its large surface area. However, the van der Waals interaction between graphene and protein molecule was found to contribute more to the denaturing effect of graphene. Based on the denaturing process of GA module, we propose that the van der Waals interaction between graphene and proteins contributed more to its toxicity.

The effect of graphene layers on free energy landscape was shown in Figure 2.5 using RMSD and radius of gyration as reaction coordinates. The presence of graphene layers affected GA module and altered its free energy landscape. GA module explored four major free energy local minima in between graphene bilayer and only one minimum in bulk water. As discussed, the free energy which made it across the barriers among these minima could be from the van der Waals interaction with graphene and solvation effect. However, the free energy landscape of protein $G$ was left unchanged indicating much higher free energy barriers of protein $\mathrm{G}$ when denaturing from native structure. 

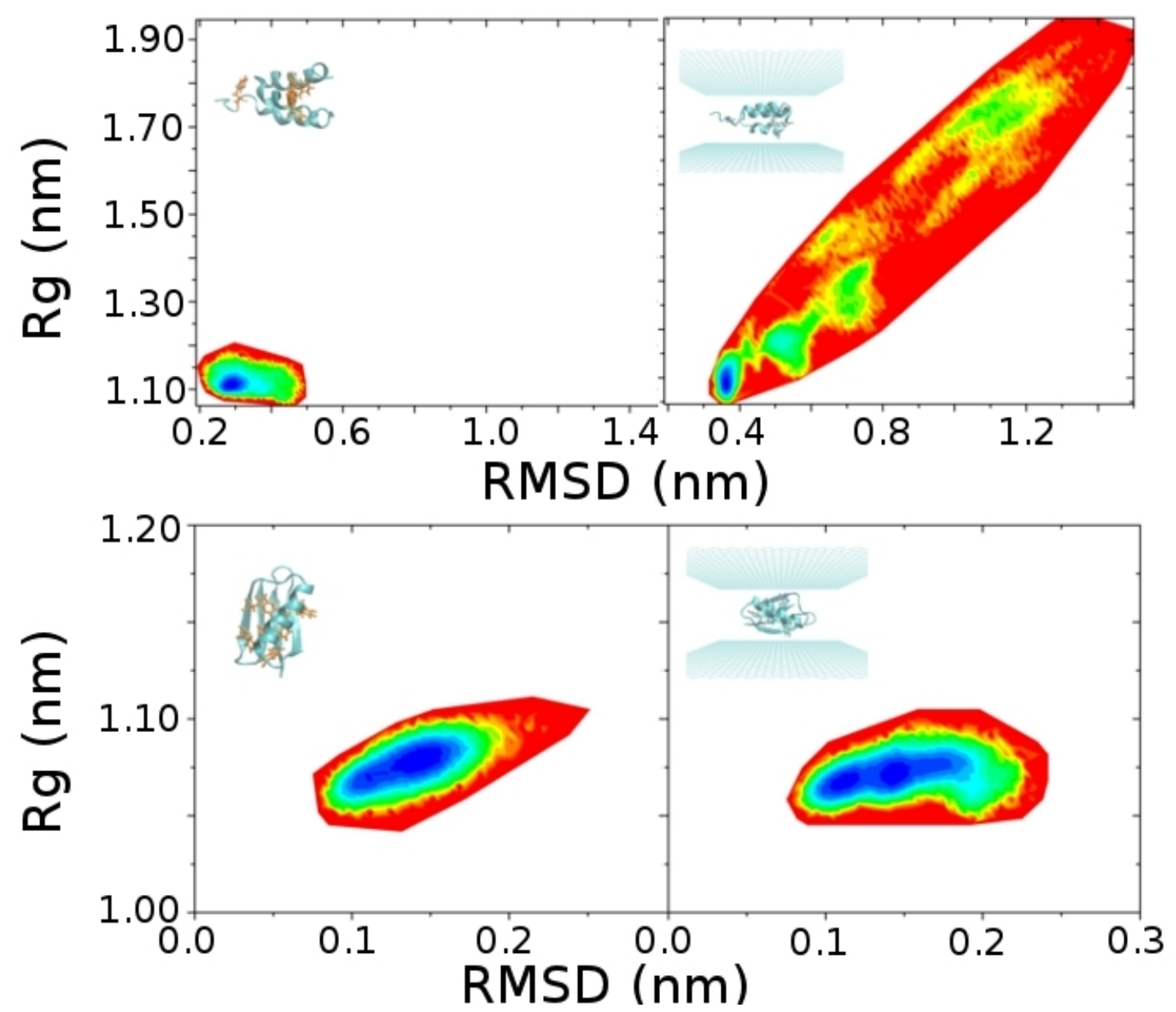

Figure 2.5: Free energy landscape obtained using RMSD and Radius of gyration in protein-graphene simulations. The free energy landscapes in the absence (left) and presence (right) of graphene bilayer are plotted. Data from the last $50 \mathrm{~ns}$ of all simulations are used. Graphene significantly altered the free energy landscape of GA module while mildly affected that of protein G. 

GRAPHENE

\section{Residue Dynamics}

During our simulations, protein molecules in between graphene layers underwent conformational changes of varying degrees: GA module loses its secondary structures while protein $\mathrm{G}$ preserves the structures very well. To further understand the physical origin of the distinctly different denaturations, the distance between the $\mathrm{COM}$ of each residue and the central plane of graphene layers is plotted in Figure 2.6. As can be seen, GA module (Figure 2.6a) shows a much less stable dynamic behaviour. In the early stage of the trajectory, the protein molecule changed its conformation as a response to graphene layers and reached a meta-stable state at about 19 ns. Four segments of protein, residues 1-9, 19-22, 33-37 and 49-53, approached to graphene surfaces and remained there in the rest of the trajectory.

On the other hand, protein $\mathrm{G}$ molecule did not present much conformational change. There is only two short part of the peptide chain close to the graphene surface, residues 9-11 and 21-22. Such small portion of near graphene residues is due to the high stability of protein $G$ molecule. As the protein $G$ molecule kept its integrity, only residues located at its surface had chance to come close to graphene surfaces. One notable difference between the distance plots of protein $\mathrm{G}$ and GA module is that, simultaneous distance change of most of protein $\mathrm{G}$ residues happened, which is reflected as a notable vertical line across the plot. To name a few, it happened at $27 \mathrm{~ns}, 37 \mathrm{~ns}$ and $75 \mathrm{~ns}$. These simultaneous distance changes demonstrate that the motion modes of protein $\mathrm{G}$ inside graphene layers are translation or rotation.

Among the naturally occurring residues, aromatic residues may play an important part in the dynamics of protein molecules in between graphene bilayer because they can form strong interactions with graphene surface. There are four aromatic residues (TRP5, PHE27, TYR28 and PHE29) in GA module and six aromatic residues (TYR3, PHE30, TYR33, TRP43, TYR45 and PHE52) in protein G. We investigate their interaction with graphene from two aspects: (1) the distance between the COM of the residues to center plane of graphene layers; (2) the acute angle between the normal of aromatic ring and $\mathrm{Z}$ axis, called $\theta$. As shown in the 
a.

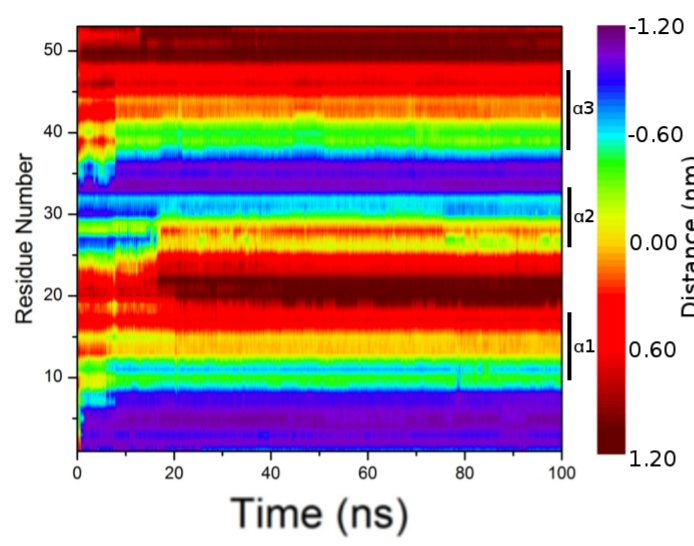

C.

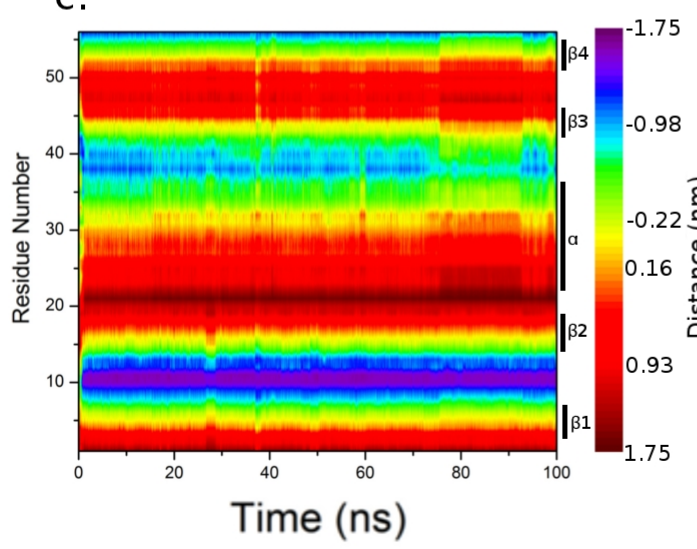

b.

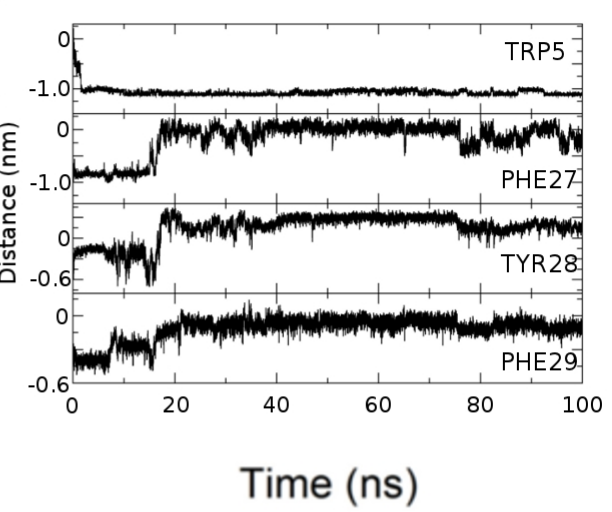

d.

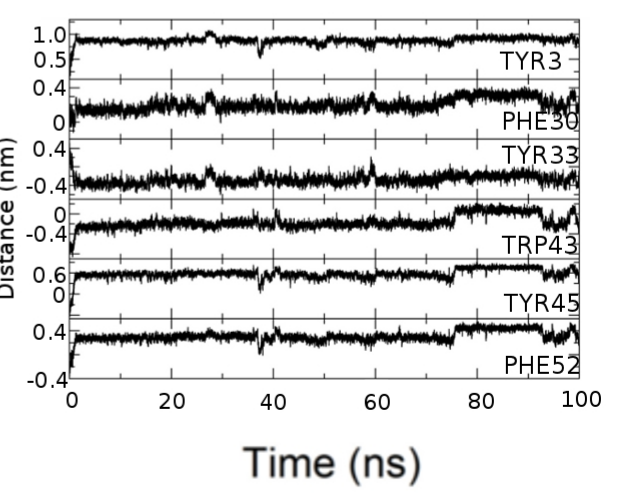

Figure 2.6: The distance between residue COMs and central plain of graphene layers. a and c depict the distance for all residues in GA module and protein G respectively. The distances for aromatic residues are plotted in b (GA module) and d (protein G). 
Chapter 2. Molecular Dynamics Study of Biomolecules Interacting with GRAPHENE

right column of Figure 6, TRP5 in GA module approached graphene layer very rapidly and maintained the close contact with graphene layer. Because TRP5 is located near the N-terminus of GA module, it is easy to get into close contact with graphene due to the flexibility of peptide end. The other three aromatic residue of GA module are consecutive to one another, which makes it hard for them to come into close contact with graphene simultaneously, even if the protein molecule is denatured. In all of our trajectories, at most one out of these three residues can obtain close contact with graphene at one time (data not shown).

In the case of protein $\mathrm{G}$, all side chains from aromatic residues are packed together tightly and buried inside the protein (see Figure 2.1). This compact structure adds significant difficulties to the aromatic residues to make contact with graphene at the same time. This can only be accomplished by destroying the integrity of protein $G$ molecule. However, residues that are near the surface of protein $\mathrm{G}$ molecule still have chances to be close to graphene layers, for example, TYR3, TYR33 and TRP43.

Another quantity which can be used to specify the interaction between aromatic residue and graphene layer is the angle between aromatic ring plane and graphene plane. Since in all of our simulations graphene layers are parallel to XY plane, we choose an equivalent angle: the acute angle $\theta$ between the normal of aromatic ring and $\mathrm{Z}$ axis to present the dynamics of aromatic rings. Small values indicate strong $\pi$-stacking interaction between aromatic ring and graphene. We counted the number of frames in which $\theta$ angle is less than 20 degrees in the last 50 ns of all trajectories, and calculated the percentage of this kind of frames in all frames. The results are presented in Table 2.1 .

As can be seen, in GA module, the flexibility of the N-terminus gives TRP5 the highest possibility to acquire a small $\theta$ angle. The consecutive position of the other three aromatic residues introduced steric resistance which makes it harder for these three residues to get a small $\theta$ angle at the same time. Presented here are lower possibilities of these three residues to parallelize with graphene surface, even in the denatured cases (separation 1 and 2). With protein G, the possibilities 
Chapter 2. Molecular Dynamics Study of Biomolecules Interacting With GRAPHENE

Table 2.1: Percentage of time where $\theta<20^{\circ}$. See text for the definition of $\theta$. Data are averaged over the last $50 \mathrm{~ns}$ of all trajectories.

\begin{tabular}{rccccc}
\hline \hline \multirow{2}{*}{ Residue } & \multicolumn{4}{c}{ Percentage (\%) } \\
\cline { 2 - 5 } GA module: & TRP5 & 94.47 & 60.81 & 53.03 & 8.14 \\
& PHE27 & 36.16 & 25.29 & 46.60 & 17.37 \\
TYR28 & 43.73 & 7.12 & 2.17 & 3.05 \\
PHE29 & 38.53 & 14.96 & 10.57 & 38.18 \\
\hline Protein G: & TYR3 & 0.00 & 0.01 & 0.03 & 22.54 \\
PHE30 & 0.00 & 11.20 & 0.01 & 6.10 \\
TYR33 & 31.82 & 35.60 & 8.49 & 8.62 \\
TRP43 & 5.24 & 3.49 & 6.76 & 0.00 \\
TYR45 & 0.01 & 21.38 & 0.00 & 0.04 \\
PHE52 & 0.00 & 0.72 & 1.91 & 1.00 \\
\hline
\end{tabular}

of small $\theta$ angle are much smaller than GA module, indicating weak interaction between the aromatic residues and graphene. It is noted that $\theta$ angle of TYR33 has a possibility of about $30 \%$ to achieve less than 20 degrees in small separations. The main reason is that TYR33 is close to protein G molecule surface (see Figure 2.1). It has more chance to have contact with graphene and to adjust its conformation.

Obviously, the aromatic residues in GA module have stronger interaction with graphene compared to the aromatic residues in protein G. The difference can be attributed to the different structural stability of these two protein molecules. The three $\alpha$-helices in GA module are packed together by hydrophobic interactions which is easier to corrupt. However, to break up the integrity of protein G molecule, not only would the hydrophobic core at the center be disrupted, but also the hydrogen bonds between $\beta$-sheets. The denaturing effects of graphene can be significantly different on the proteins with different structural characteristics. It may cause total denaturation of some protein, or not much changes to other proteins. Its relationship with protein structure, and maybe amino acid residue sequence, is subjected to further study. 


\subsubsection{Summary}

In order to study the direct impact of a graphene bilayer on protein molecules, a series of molecular dynamics simulations were conducted. We chose two protein molecules with dissimilar structure characteristics to build our simulation systems. The influence of a graphene bilayer can be significantly different on different protein molecules. Our simulations exhibited a significant denaturation of a 3 - $\alpha$-helix bundle GA module and a great structure preservation of a $\alpha / \beta$ hybrid protein G. Additionally, we found a correlation between the separation of graphene layers and the extent of GA module denaturation, which is smaller separation can cause greater denaturation. Though protein $\mathrm{G}$ became slightly more flexible between graphene layers than in pure water, it retains its native structure quite well during all of our simulations.

Energetic analysis showed that van der Waals interaction played a greater role in the denaturing of GA module. Meanwhile, solvation free energy showed a facilitating effect on the denaturing. The van der Waals interaction between protein $G$ and graphene layers is also strong. However, protein G showed its property of keeping its integrity under the influence of graphene layers.

The influence of graphene on different proteins is different. To construct a relationship between the stability of protein and its structure when interacting with graphene, further studies should be done, and more data are needed. Yet, we can conclude that van der Waals interaction should not be overlooked when the protein-graphene complex is constructed. In our modeled system, the magnitude of van der Waals energy change between protein molecules and graphene layers is several times greater than solvation free energy change. This factor should be considered when one is trying to realize any biological applications of graphene and its derivatives. 


\subsection{Short dsDNA Sandwiched Between Graphene Layers}

Graphene functionalization with DNA is attempted in the last few years. $.57 / 80] 82$ Due to the $\pi$ electrons possessed by both DNA bases and grahene, functionalized graphene with DNA can present interesting electronic or optical features, which is useful in many cases. For instance, graphene functionalized with ssDNA can be used as transistor for lable-free detection of complementary ssDNA segments. ${ }^{[57}$ As before, the interaction between DNA duplex and graphene layers is studied with all-atom MD simulations, to illustrate detailed events happened at the graheneDNA interface. To simplify the simulation, and to achieve a biologically meaningful simulation scale, simple force filed model is used in this study.

\subsubsection{Introduction}

Owing to its unique structural, optical and electronic properties, $\frac{83184}{\text { graphene has }}$ been widely researched for various potential applications in the biomedical and biological fields. Its fluorescence quenching properties has shown huge capability in biosensing to detect and diagnose diseases. ${ }^{82}$ Its ability to differentiate stem cells ${ }^{85186}$ has also aroused interest in stem cell research, in hope of combating diseases and creating regenerative medicine. Another potential application is to be used to sequence DNA. $\stackrel{87}{ }$ The unique electrical signal from the fluctuation of electronic conductivity as DNA passes through graphene nanopore could be used to differentiate nucleotides. To take full advantage of DNA functionalized graphene, it is of great interest in exploring the interaction between graphene and DNA.

Despite all the application studies, the basic theory of DNA-graphene interaction is always of great interests. Experimental studies have shown that DNAs were able to self-assemble on graphene. $\sqrt[88 \mid 89]{ }$ For example, a suspension of graphene-DNA hybrids can be obtained by ultrasonication of graphene and ss-DNA in an aqueous solution, followed by centrifugation. ${ }^{89}$ Chemically oxidized graphene oxide that was reduced by hydrazine in the presence of ss-DNA was stable in sedimentation 
Chapter 2. Molecular Dynamics Study of Biomolecules Interacting with GRAPHENE

for months. It was believed that ss-DNA played an important role in stabilizing reduced graphene oxide solution by adsorbing onto the graphene layers.

In a recent study, it was found that dsDNAs were able to be trapped between two graphene layers. ${ }^{90} \mathrm{dsDNAs}$ trapped between two graphene layers would experience different impact originated from graphene layers, comparing to when dsDNAs are adsorbed onto a graphene surface. Different interaction may result in different dynamics. Atomic simulations of DNA-graphene system have been done to study details upon DNA adsorption. In a simulation study previously conducted by Zhao, 91 dsDNAs were initially placed parallel to a graphite surface consisting of five graphene layers stacked one on top of another. It was found that they can adopt two different self-assemblies, dsDNA perpendicular to the graphite layer ("standing up" position) or parallel to the graphite layer ("lying down" position). Therefore, it is of great interest and importance to explore the self-assembly of dsDNAs trapped between graphene layers at atomic level. It is also interesting to study the DNA-DNA interaction upon adopting their final conformations when multiple dsDNA molecules are trapped between graphene layers.

In this study, we hope to gai insights on the dynamics of dsDNAs trapped between two graphene layers through means of molecular dynamics simulation. The interaction between dsDNAs separated by a graphene layer was also examined. Graphene bilayer system was employed in this study to mimic the experiment where the dsDNAs are trapped between graphene layers. The dynamics will be analyzed using twenty dsDNAs in graphene bilayer systems 1 to 4 as shown in Figure 2.7. dsDNAs are numbered for easy identification. Interaction separated by a graphene layer will be analyzed using dsDNAs in graphene bilayer systems 3 and 4 as shown in Figure 2.7 .

\subsubsection{Computation Method}

The interaction between dsDNAs and graphene was examined by means of allatom molecular dynamics simulation. The system model was built under the AMBER99SB ${ }^{63}$ force field and with TIP3P ${ }^{64}$ water model. The dsDNA molecule subjected to simulation has 10 base pairs with the sequence of 5'-(AAAAAAAAAA)-3' 
Chapter 2. Molecular Dynamics Study of Biomolecules Interacting with GRAPHENE

(and its complementary strand), and modeled in standard B-DNA conformation at the beginning. A labeled picture of a dsDNA molecule is shown in Figure 2.7. At the beginning of all simulations, dsDNA molecules were put between graphene layers with their axes parallel to graphene surfaces. The graphene layers were modeled as infinite planes by creating bonds across the periodic box boundaries, as in previous protein-graphene study. The atom type CA of AMBER99SB force field (C-C bond length $1.40, \sigma=0.3400, \epsilon=0.3598$, zero charged) was adopted for graphene carbon atoms in all simulations. Graphene and dsDNA molecules were put into a triclinic box with periodic boundary condition applied to all directions. The graphene layers were parallel to XY-plane of the periodic box.

A total of four graphene bilayer systems 1 to 4 were prepared for the simulation as shown in Figure 2.7. The twenty dsDNA molecules were numbered for easy identification. The initial distance between two adjacent graphene layers is 3.8 $\mathrm{nm}$, which is roughly the length of the DNA molecule. Graphene layers are at least $1.0 \mathrm{~nm}$ away from the box edge in $\mathrm{Z}$ direction. Then, the box was soaked in TIP3P water. ${ }^{64}$ Sodium ions were added into the box to balance the negative charge introduced by dsDNA molecules.

After preparing the starting structures of all simulations, they are subjected to an energy minimization step followed by a 100 ps constant volume and constant temperature (NVT) simulation, at $300 \mathrm{~K}$. All heavy atoms in graphene and dsDNA were held restrained. After the NVT simulation, a 2 ns long isothermal-isobaric (NPT) equilibrium phase was performed to equilibrate all systems at $300 \mathrm{~K}, 1$ bar. Since we are using a periodic molecule model for graphene layer, vector scaling resulting from pressure coupling could cause unnecessary and violent stress along graphene plane, which could cause simulation crash. To achieve an NPT ensemble, we coupled pressure with a one-dimensional scaling in the $Z$ direction. DNA heavy atoms were also restrained in this phase. Following the equilibration, production simulations were performed with no restraints under a constant temperature (300 $\mathrm{K}$ ) and constant pressure (1 bar) condition under the control of Parrinello-Rahman barostat $\frac{69170}{}$ and velocity rescaling thermostat. 68 The pressure coupling was done 

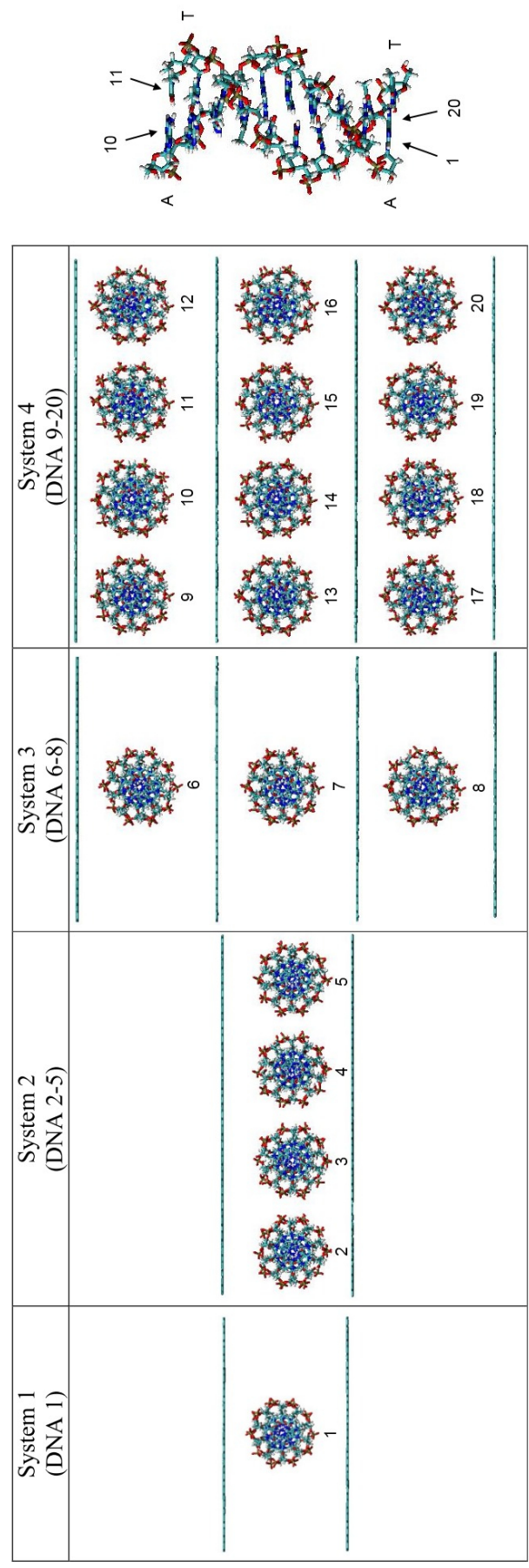

Figure 2.7: DNA-graphene simulation setups. Short DNA duplexes are placed inside graphene layers. The axes of DNA molecules are parallel to graphene surface. DNA molecules are numbered for easy indentification. 
Chapter 2. Molecular Dynamics Study of Biomolecules Interacting with GRAPHENE

by coupling to only $Z$ direction, as in the protein graphene simulations done in the previous section. The production simulation of each case was performed for 100 ns. The integration time step for all simulations was 2 fs. All bonds containing hydrogen atoms were constrained using LINCS ${ }^{67}$ algorithm. All simulations were performed with GROMACS simulation package version 4.5.4. ${ }^{[73}$ All the analysis of dsDNAs was done with GROMACS ${ }^{173}$ utilities and VMD. ${ }^{65}$ All the water molecules and ions were removed from the pictures to facilitate visualization.

\subsubsection{Results and Discussion}

\section{Self-Assembly of dsDNAs in Graphene Bilayer System}

To better understand dynamics of dsDNA between graphene layers, double-stranded oligo(A)-oligo(T) DNAs were used in the graphene bilayer system. All twenty dsDNAs from four different graphene bilayer systems 1 to 4 were used for analysis. On close examination of the simulation trajectories, it was found that all dsDNA molecules took "stand-up" position, i.e. the axes of dsDNS molecules are nearly perpendicular to graphene surfae, after a very short simulation period. Within the space between two graphene layers, dsDNA molecules were assembled in a regular arrangement. We call this process the self-assembly of dsDNA molecules, since this arrangement is preferred by dsDNA molecules. The self-assembly exhibits two possible sequential phenomena, disruption of hydrogen bond between nucleotides and base stacking interaction between the unpaired nucleotides. Each phenomenon will be described in greater details later.

The self assembly begins with the rotation of the dsDNA followed by the subsequent approach of the terminal nucleotides of the dsDNA towards the graphene surface. The relative angle $\theta$ (depicted in Figure 2.8 ) between the principle axis of the dsDNA and the normal of the graphene surface was used to monitor the rotation. The full rotation of the dsDNA can be depicted by the cosine value of angle $\theta$ from 0 to 1 . The change in the cosine value of angle $\theta$ over a period of time for DNA 1 is plotted in Figure 2.8 a. Initially, the angle $\theta$ fluctuated randomly. The cosine of $\theta$ gradually approached 0.966 radians. After $4.43 \mathrm{~ns}$, the cosine of 
Chapter 2. Molecular Dynamics Study of Biomolecules Interacting with GRAPHENE

$\theta$ for DNA 1 stabilized and maintained at about 0.966 radians throughout the simulation time. This suggests that the approach of the terminal nucleotides towards the graphene layers above and below it was established. The deviation of $\cos \theta$ from 1 radians was due to the not perfect matching in the distance between graphene layer and the length of the dsDNA. The change in angle $\theta$ over a period of time for the remaining dsDNAs in system 2, 3 and 4 were also plotted in Figure $2.8 \mathrm{~b}, 2.8 \mathrm{c}$ and $2.8 \mathrm{~d}$ respectively.

The time at which all dsDNAs achieved the vertical conformation is plotted in Figure 2.8f. It was observed that all dsDNAs were able to rotate themselves to the upright conformation. By placing dsDNAs in a bilayer, most dsDNAs were able to rotate from the initial "lying down" conformation to the upright conformation within 4 ns. In a previous study conducted by Zhao, ${ }^{91} \mathrm{dsDNAs}$ were allowed to self assemble on a graphite surface from the "lying down" conformation to the upright conformation. It took the dsDNAs about 30 ns to self assemble on the graphite surface. In comparison, dsDNA trapped in graphene bilayer were able to achieve such conformation about 7.5 times faster. The increasing in the formation of the self assembly may be due to the increase in the van der Waals interaction between the dsDNA and the graphene layers.

Yet, there are also some dsDNAs that took longer to rotate to the upright conformation, such as DNA 3, 18 and 19. Up to three hydrogen bonds between the terminal base pair of DNA 3 were broken during the process of rotation. Hydrogen bonds are important in maintaining the helical structure of DNA. Too much hydrogen bond disruption in the structure can cause the dsDNA to become too floppy and might result in difficulty to rotate the dsDNA. DNA 18 and 19 are next to each other initially. During the simulation, the terminal nucleotides of DNA 18 were observed to have a contact with the terminal nucleotides of DNA 19 from $0.6 \mathrm{~ns}$ to $7.6 \mathrm{~ns}$. During the approach of the nucleotides to the graphene surface, many hydrogen bonds were also broken, causing deformation of the dsDNA. As a result, both DNAs took longer time to rotate. 
Chapter 2. Molecular Dynamics Study of Biomolecules Interacting with GRAPHENE
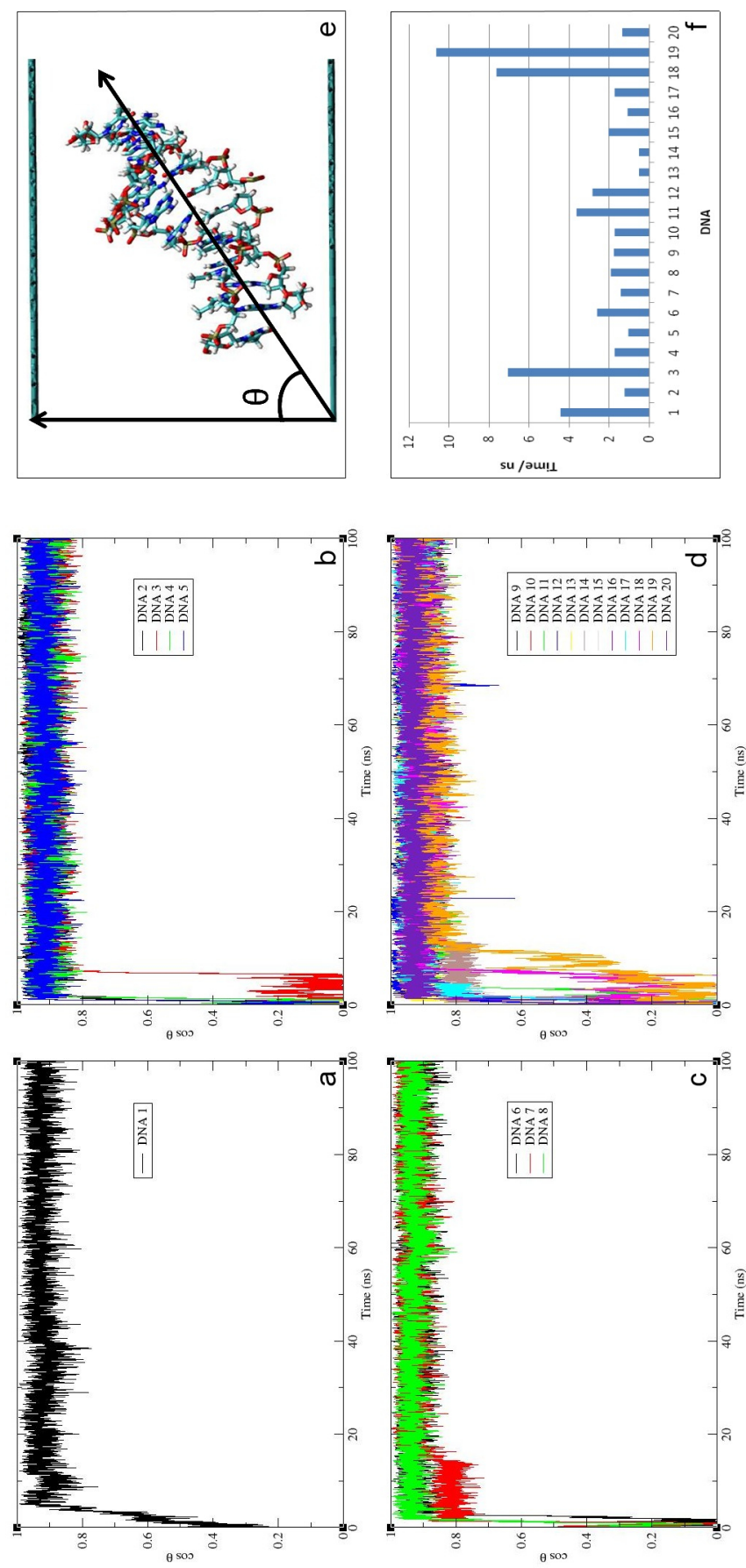

Figure 2.8: DNA orientation as a function of time. The angle $\theta$, as defined in e, is used to monitor the orientation of DNA duplex. $\cos \theta$ of all DNA duplexes are plotted in a-d. Time to achieve "upright" orientation for all DNAs is shown in $\mathrm{f}$. 
Chapter 2. Molecular Dynamics Study of Biomolecules Interacting with GRAPHENE

During the approach of dsDNA to graphene surface, the hydrogen bond of the approaching base pairs could be broken before contact and reform after full contact with the graphene surface or could remain unbroken throughout the approach. In order to monitor the formation and breaking of hydrogen bond at the approaching base pair, the number of hydrogen bond between the two ending base pairs during the simulation was used. To define the hydrogen bond between the hydrogen bond donor and acceptor, the maximum distance between the donor and acceptor was taken to be $3.5 \AA$ and largest angle of acceptor-donor-hydrogen to be $30^{\circ}$.

As there are two ends of the dsDNA that are in contact with the graphene surface, there are 40 separate case studies for the hydrogen bond analysis. Also, from Figure 2.8f, it was observed that most dsDNA achieved full rotation to the vertical conformation within $10 \mathrm{~ns}$. Hence, hydrogen bond of the base pairs were analyzed up to $10 \mathrm{~ns}$. The number of hydrogen bonds existing between base pair 1 and 20 and between base pair 10 and 11 of DNA 1 during the rotation process are shown in Figure 2.9 and 2.9 b respectively. It was observed that the hydrogen bond between base pair 1 and 20 initially started at two and fluctuated around one and two. Despite the fluctuation, it was able to maintain the hydrogen bond till 10 ns. The hydrogen bond between base pair 10 and 11 initially started at two and fluctuated between one and three. It dropped to zero at 2.38 ns. Hydrogen bond was subsequently reformed at 4.43 ns when the number of hydrogen bond was raised back to two and was maintained till 10 ns. Hence, base pair 10 and 11 of DNA 1 experience disruption and subsequent reform of hydrogen bond, while base pair 1 and 20 of DNA 1 did not experience any disruption of hydrogen bonds.

A plot of the occurrence of hydrogen bond disruption is plotted in Figure 2.9c and $2.9 \mathrm{~d}$ for the two different ends of the $20 \mathrm{dsDNAs}$. Hydrogen bond disruption between the base pair is represented by 0 , while no hydrogen bond disruption is represented by 1 . Based on Figure 2.9k and 2.9d, the chance of hydrogen bond disruption is 28 out of 40 occurrences, $70 \%$. This suggests that the terminal base pair of dsDNA have a tendency to cleave their hydrogen bonds before making 

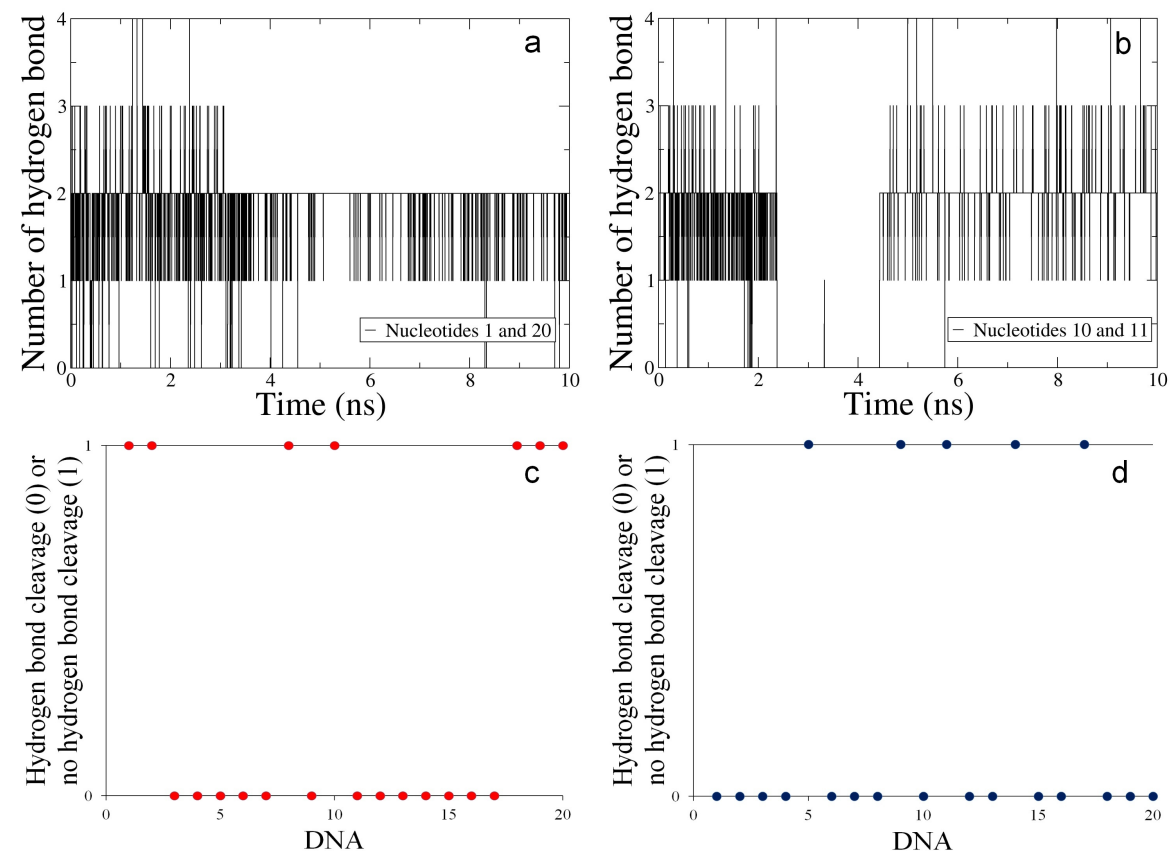

Figure 2.9: Inter-chain hydrogen bond number between ending bases as a function of time. Number of hydrogen bonds between (a) Nucleotides 1 and 20. (b) Nucleotides 10 and 11 of DNA 1. A plot of the occurrence of hydrogen bond disruption (denoted as 0 ) or no disruption (denoted as 1) for 5' end (c) of DNA 1 to 20 and 3' end (d)of DNA 1 to 20. 
Chapter 2. Molecular Dynamics Study of Biomolecules Interacting with GRAPHENE

contact with the graphene surface, which could be due to the strong $\pi$ stacking interaction between the base pairs and the graphene surface.

After the hydrogen bond disruption of the terminal base pair, the two nucleotides can form either $\pi$ stacking interaction with the graphene surface or base stacking interaction with one another. The $\pi$ stacked formation with the graphene surface or the base stacked formation between the two unpaired nucleotides was monitored using the distance between the individual terminal nucleotide and the graphene surface. After $\pi$ stacking with the graphene surface, the distance between the nucleotide and the graphene surface is about $3.5 \AA$. Base stacked formation will result in a plateau to be observed at the distance of about $6.5 \AA$ between the nucleotide and the graphene surface. An illustration of base stacked formation is shown in Figure 2.10a. After some time, base stacking between the nucleotides will be disrupted and $\pi$ stacking of both terminal nucleotides with the graphene surface will be formed.

The distance of base pair 1 and 20 of DNA 1 with the graphene surface over a period of time is plotted in Figure 4b, while the distance of base pair 10 and 11 of DNA 1 with the graphene surface over a period of time is plotted in Figure $2.10 \mathrm{k}$. From Figure $2.10 \mathrm{~b}$, it was observed that base pair 1 and 20 did not form any base stacking interaction between them. Instead, the two nucleotides form $\pi$ stacking with the graphene surface together. In Figure 2.10k, nucleotide 11 that is nearer to the graphene surface formed a $\pi$-stacked interaction with the graphene surface, after its hydrogen bond with nucleotide 10 was cleaved at 2.9 ns. This is represented by the distance of about $3.5 \AA$ between nucleotide 11 and the graphene surface. The unpaired nucleotide 10, then stacked on top of its counterpart, nucleotide 11. This is represented by the distance of about 6.5 $\AA$ between nucleotide 10 and the graphene surface. The base stacking was later disrupted at $4.43 \mathrm{~ns}$ and $\pi$ stacking interaction was formed between nucleotide 10 and the graphene surface.

A plot of the occurrence of base stacking interaction between nucleotides is plotted in Figure 2.10d and 2.10 for both sides of the 20 dsDNAs. Absence of 

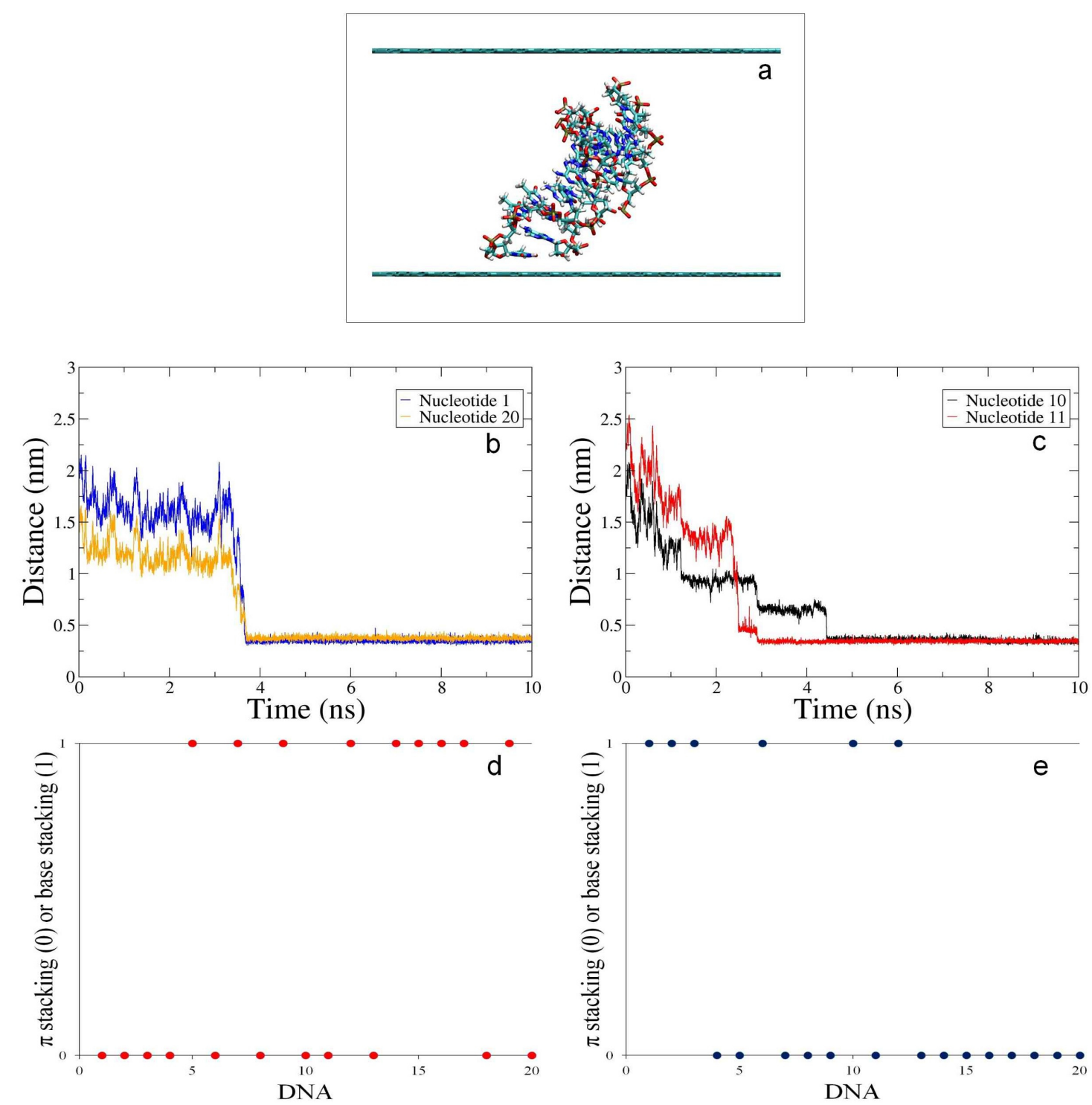

Figure 2.10: $\pi$ stacking interactions between DNA and graphene. (a) Base stacking conformation between two nucleotides. Distance of nucleotides of DNA 1 with graphene surface. (b) Distance between nucleotides and graphene surface of nucleotides 1 and 20, showing typical adsorption without hydrongen bond disruption. (c) Similar to b but for nucleotides 10 and 11, showing adsorptoin process with hydrogen bon disruption. A plot of the occurrence of base stacking (no base stacking is represented by 0 , and 1 otherwise for 5' (d) and 3' (e) ends of all DNA duplexes. 
Chapter 2. Molecular Dynamics Study of Biomolecules Interacting with GRAPHENE

base stacking between the nucleotides and the graphene surface is represented by 0 , while base stacking between nucleotides is represented by 1 . From Figure $2.10 \mathrm{~d}$ and 2.10 , the chance of forming base stacking between nucleotides is 15 out of 40 occurrences, $37.5 \%$. This suggests that the terminal nucleotides of dsDNA might not have base stacking between nucleotides readily. This could be due to the high torsion energy introduced on the dsDNA backbones by the base stacked formation. Hence, such base stacked formation might be unfavorable, as depicted by the low occurrence.

\section{Interaction between DNAs separated by a graphene layer}

The backbone of dsDNAs is lined up with negatively charged phosphate groups. As a result, dsDNAs placed in a graphene bilayer are less likely to be attracted to one another due to the electrostatic repulsion from the phosphate backbone. This was confirmed by our results (data not shown). Negligible van der Waals interaction was observed between dsDNAs sandwiched between graphene bilayer.

Examination with system 3 was performed to investigate the interaction between dsDNA separated by a layer of graphene. The interaction between dsDNA was monitored with the van der Waals energy between them. The van der Waals interaction energies between DNA 6 and DNA 7 and between DNA 7 and DNA 8 were plotted in Figure 2.11a. Dips in interaction energy up to $-10 \mathrm{~kJ} / \mathrm{mol}$ between DNA 6 and 7 as shown in Figure 2.11a were observed. These dips were observed throughout the simulation, suggesting that DNA 6 and 7 were able to interact with one another. Similar interaction between DNA 7 and 8 was also observed. However, DNAs 6, 7 and 8 were not aligned together. This might indicate that the van der Waals interaction between them might be too weak for alignment.

System 4 was utilized to observe the effect of increasing number of dsDNAs in the bilayers on the van der Waals interaction between dsDNAs. van der Waals energy was used to monitor the interaction between dsDNA separated by a graphene layer. For simplicity, only interactions observed between the top and middle layers will be displayed. The van der Waals interaction energies between DNAs 13, 14, 

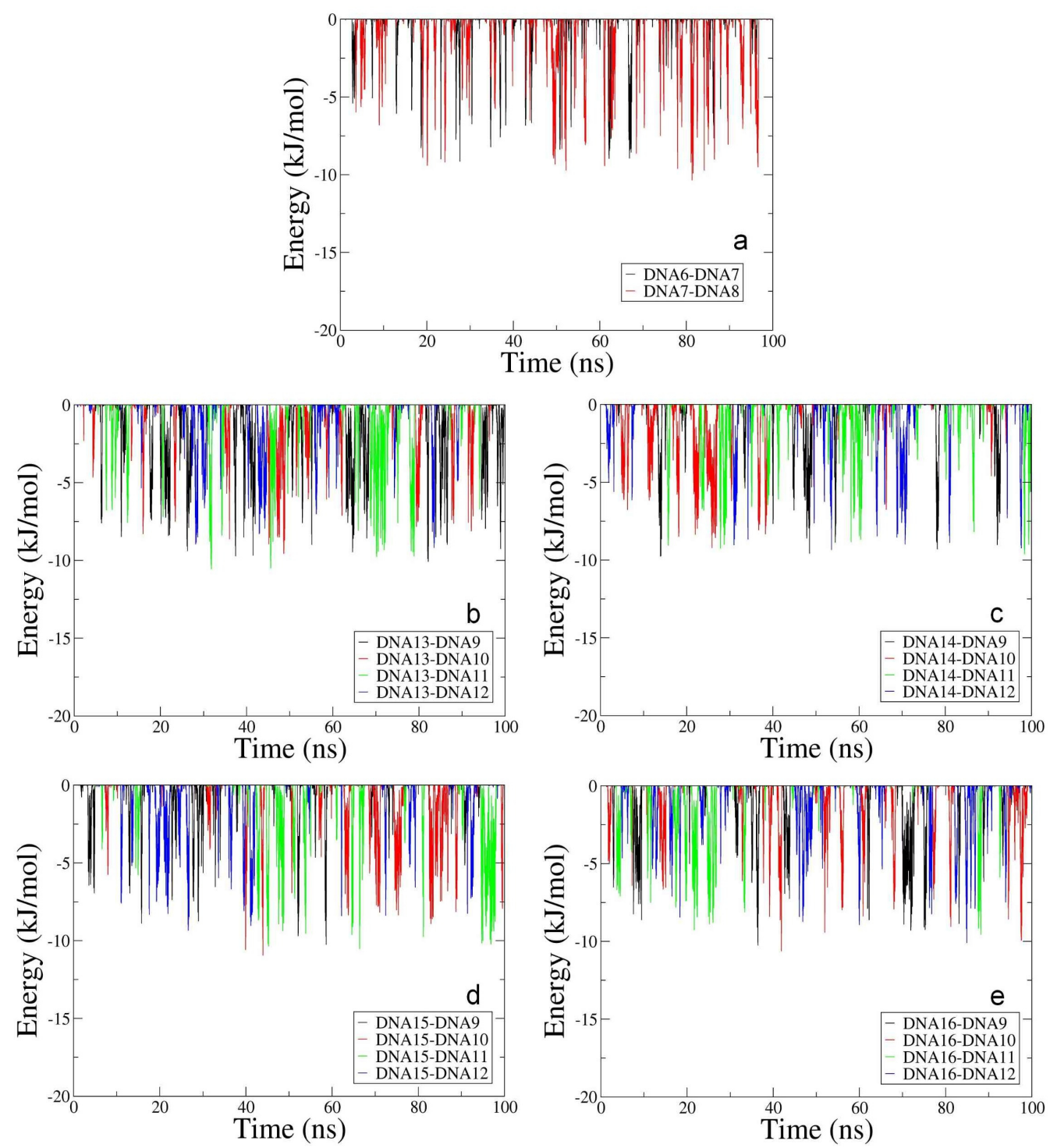

Figure 2.11: Cross-graphene interactions of DNA. van der Waals interaction energy between DNA separated by graphene layer. 
Chapter 2. Molecular Dynamics Study of Biomolecules Interacting with GRAPHENE

15 and 16 and the four dsDNAs in the top layer were plotted in Figure 2.11b, c, $\mathrm{d}$ and e respectively. Dips in interaction energy up to $-10 \mathrm{~kJ} / \mathrm{mol}$ between DNA 13 and the four DNAs in the top layer as shown in Figure 2.11b were observed. The interaction between DNA 14, 15 and 16 and the four dsDNAs in the top layer was also observed of such behavior. For the remaining dsDNAs in system 4, similar analysis was done. It was found that the remaining dsDNAs in system 4 also seemed to follow this trend. These energy dips are similar to those observed in system 3. This suggests that the number of dsDNAs in the bilayer will not influence the van der Waals interaction between DNAs separated by a graphene surface.

\subsubsection{Summary}

In summary, this study provides an insight on the molecular dynamics of dsDNA sandwiched in between graphene bilayers. Within graphene bilayer, no lying down conformation was observed after $10 \mathrm{~ns}$ compared to the self assembly of dsDNA on a graphite layer. dsDNA trapped in graphene bilayer system can achieve the upright conformation from the initial 'lying down' position in about 4 ns. dsDNAs display two possible sequential phenomena to achieve the upright conformation. Terminal nucleotides of dsDNA could cleave or maintain the hydrogen bond between them. Following that, the nucleotides could form $\pi$ stacking interaction with the graphene surface or base stacking interaction with opposed base followed by $\pi$ stacking interaction with the graphene surface. Interaction between dsDNAs separated by graphene layer is weak with occasional energy dips up to $-10 \mathrm{~kJ} / \mathrm{mol}$. The number of dsDNAs in the bilayer did not affect the interaction between dsDNAs separated by a graphene layer.

\subsection{Concluding Remarks}

In this chapter, all-atom molecular dynamics simulations are carried out to study interactions of graphene with biomolecules, i.e. protein and DNA. Atomic details 
Chapter 2. Molecular Dynamics Study of Biomolecules Interacting with GRAPHENE

are shown upon the adsorption of biomolecules upon graphene. Due to the planar structure and high density of graphene, the interactions turn out to be nonspecific and fairly strong. Generally, biomolecules experience structural deformation upon adsorption, i.e. the denaturation of protein and broken base pairs at the end of DNA duplex, due to the strong interaction. These observations are useful to further our understanding of grephene interaction with biomolecules. It also provides guidance to graphene functionalization and application studies.

Although details are described in current study, the significance of these studies is hindered by several limitations. First of all, the graphene model in current study is not accurate and detailed enough to represent all the unique electronic properties of graphene. Graphene layers are modeled as simple Lenard-Jones particles connected by harmonic bonds. Only rough and averaged interaction can be calculated with such simple model. Other interesting electronic features of graphene, e.g. electronic conductivity and optical properties produced by conjugated $\pi$ electrons, cannot be illustrated with such model. The same applies to biomolecules. It is clear that the functionalization of graphene by biomocules is coupled with the interplay of $\pi$ conjugation on both biomolecule and graphene. More detailed model is needed to study these systems in the long run.

It is believed that all the interesting properties presented by graphene are related to $\pi$ electron conjugation. Traditional force fields are inadequate to model $\pi$ electron, as well as interactions mediated via $\pi$ electron. One way of improving current force field to meet these needs is to include polarization terms into the energy expression of force fields. Numerous models are attempted to perfect force fields, and not to introduce too much complexity and computation at the same time. In the following chapters, several improved models are applied to protein molecules, as an attempt to improve the performance of force field in MD simulations. As always, good models are meaningful to studies of more complex systems, such as systems interesting in biomaterial and biotechnology. 


\section{Chapter 3}

\section{Backbone Fluctuating Charge Model}

In this chapter, a polarization model called backbone fluctuating charge model has been explored. The model has a peculiar origin. As known to all, peptide or protein backbone plays an important role in forming the native structure, as well as in protein folding. Therefore, it is believed that including polarization effect on protein backbone can improve force field performance, at least to some extent. Apparently, it compromises between physical reality and computation resource consumption. Although, it is physically ill-defined in the context of molecular dynamics simulation, several attempts have been realized to explore the idea. Here, a comparison among two such kind of models and AMBER force field is presented, in the hope that some evidence could be presented to support or undermine this backbone fluctuating charge idea.

\subsection{Introduction}

Conventional molecular dynamic (MD) simulation relies on force fields to describe molecular interactions. Second generation force fields (e.g. AMBER,, 1819 CHARMM, ${ }^{20}$ GROMOS ${ }^{22}$ etc.) have acquired tremendous success in simulations of various kinds of molecules, especially with proteins and peptides. In these force fields, it is common to divide molecular interactions into two sorts, bonded and 
non-bonded interactions, as non-bonded interaction is represented by a van der Waals term and an electrostatic term, as discussed in Chapter 1. To preserve simplicity, van der Waals and electrostatic parameters are usually fixed, lacking the ability of describing instant polarization interactions. This methodology is adequate in most scenarios. Though static parameter can largely simplify the problem and can produce good results most of the time, polarization effect has been proved to play an important role in condensed phase simulations. Hence polarizable force field is catching up more and more attention. Generally, polarizable force fields give more accurate molecular interaction energies, hence better dynamic behaviours of interested molecules, by introducing other energy terms that are related to polarization, e.g. dipole. ${ }^{30}$ However, the development of polarizable force field takes much more effort since more variables are to be included in the equation and more parameterization work is to be done. Furthermore, introducing terms that account for polarization doesn't change the empirical nature of force fields. Regarding the performance, it is reported simulations with polarizable force field take about 4 times CPU cycles more than simulations with non-polarizable force fields, $\frac{37}{37}$ although this would not be a serious problem with the increasing computing power.

With the importance of polarization in mind, two peptide backbone charge variation models, namely polarizable hydrogen bond (PHB) $)^{27}$ and semifluctuating charge (SFC), ${ }^{92}$ were developed, compromising between accuracy and efficiency. Considering the significance of backbone hydrogen bond (Hbond) in maintaining protein structure, these two models are expected to give better description of backbone Hbond, hence better dynamic behaviour, of protein or peptide molecules. They both include polarization effect by varying atomic charges on backbone N-H and $\mathrm{C}=\mathrm{O}$ groups periodically, though the philosophies behind are different.

Although some success has been achieved by both PHB and SFC models, it is still not clear whether they are good for general protein simulations. Or, more generally, is this compromise of just varying charges of backbone Hbond forming groups good or not? Here, we provide a more thorough case study with both 
PHB and SFC models, and try to find answer to the abovementioned question. Specifically, we run simulation on three peptides in explicit water model (see Table 3.2.3), including two $\beta$-hairpin peptides and an $\alpha$-helix peptide. All simulations are started from their native states (NMR structures in PDB). Multiple trajectories with different starting velocities are obtained to improve sampling efficiency. Structural and thermodynamical properties of all charge models are compared. Comparison to AMBER99SB force field is also included to validate the improvement with respect to traditional force field.

\subsection{Simulation Method}

\subsubsection{Polarizable Hydrogen Bond model}

In PHB model, $\stackrel{[27}{2}$ the polarization effect acting on one Hbond forming group, originated from the other Hbond forming group, was modeled. It was realized by a pre-fitted analytical formula. Gao et.al. ${ }^{27}$ used two alanine residues which form a Hbond as a model system. Atomic charges of $\mathrm{C}=\mathrm{O}$ and $\mathrm{N}-\mathrm{H}$ groups were refitted using standard procedure while varying hydrogen bond donor-accpetor distance. Charges of other part of the model system were kept same as AMBER. All acquired $\Delta q$ values were used to fit a simple exponential function of $\Delta q$, the fluctuating charge, with respect to Hbond length, for $\mathrm{C}=\mathrm{O}$ and $\mathrm{N}-\mathrm{H}$ bonds respectively. For $\mathrm{C}=\mathrm{O}$ bond, the form of the function is ${ }^{27}$

$$
\Delta q_{O}=-0.250 \exp [-0.466(R-0.623)]
$$

and for N-H bond,

$$
\Delta q_{N}=-0.843 \exp [-0.455(R+1.18)]
$$

where $R$ is hydrogen bond donor-acceptor distance.

Then the fitted exponential function was used in MD simulations in an onthe-fly manner, i.e. checking Hbond length and updating backbone $\mathrm{N}-\mathrm{H}$ and $\mathrm{C}=\mathrm{O}$ 
charges with fitted function discretely. Apparently, the whole polarization effect is incorporated in the simple formula governing backbone atomic charges. In simulations later, the PHB model gave better folding time estimation in the replica exchange molecular dynamic (REMD) simulation of a short helix.

\subsubsection{Semi-fluctuating Charge model}

Another similar backbone charge variation model, developed by Ji et.al., ${ }^{92}$ is called semifluctuating charge model, which is derived from a utterly different perspective. Ji et.al. considered the solvation process of model molecules (e.g. acetone molecule to model $\mathrm{C}=\mathrm{O}$ group). By examining ab initio data from thousands of molecular configuration, they discoverd the relation between distortion energy $\Delta E$ and dipole moment change along $\mathrm{C}=\mathrm{O}$ or $\mathrm{N}-\mathrm{H}$ bond can be fitted with a quadratic function.

$$
\Delta E_{\text {distortion }}=k\left(\mu_{\text {liquid }}-\mu_{\text {gas }}\right)^{2}
$$

Then the scenario was simplified to $\mathrm{C}=\mathrm{O}$ or $\mathrm{N}-\mathrm{H}$ group in electrostatic field. The system energy in this case can be written as

$$
E=E_{\text {self }}+E_{\text {ele }}=\left[E_{0}+k\left(\mu_{\text {liquid }}-\mu_{\text {gas }}\right)^{2}\right]+\left[q_{C} \Phi_{C}+q_{O} \Phi_{O}\right]
$$

for $\mathrm{C}=\mathrm{O}$ bond. With the simple model of charge fluctuating between $\mathrm{C}$ and $\mathrm{O}$ or between $\mathrm{N}$ and $\mathrm{H}$ atoms, we have

$$
\begin{gathered}
\mu_{\text {liquid }}-\mu_{\text {gas }}=\Delta q d_{C O} \\
q_{C}=q_{C}^{0}+\Delta q \\
q_{O}=q_{O}^{0}-\Delta q
\end{gathered}
$$

for $\mathrm{C}=\mathrm{O}$ bond, for example, where $d_{C O}$ is $\mathrm{C}=\mathrm{O}$ bond length. Plugging the above three equation into equation 3.4 , then minimizing $E$ by

$$
\frac{\partial E}{\partial \Delta q}=0
$$


we can get

$$
\Delta q=\frac{\Phi_{O}-\Phi_{C}}{2 k_{C O} d_{C O}^{2}}
$$

for $\mathrm{C}=\mathrm{O}$ bond. The same derivation can be used to determine $\Delta q$ for $\mathrm{N}-\mathrm{H}$ bond.

Hence, they related atomic charge variation with local electrostatic potential generated by environmental molecules through a regression of distortion energy with respect to dipole difference of the model molecule, so that the polarization effect acting on backbone Hbond forming groups, as well as the response can be easily obtained from their local electrostatic potential. After the parameterization, a test MD run showed better performance in distinguishing wild type and mutated WW domain protein. Since these two models both do not require heavy computation, they only consume a little more CPU time than normal simulations, e.g. SFC simulation consumes about $1 \%$ more CPU time than conventional MD.

\subsubsection{MD simulation}

In order to examine the performance of both $\mathrm{PHB}^{27}$ and $\mathrm{SFC}^{92}$ on peptide native state simulation, three small peptides are chosen as the subjects to perform MD simulations on at their native states, i.e. starting from their NMR structure. Although the number of obtained trajectories is not exactly the same for each peptide (see Table 3.2.3), a same simulation protocol is followed in all cases. First, the starting structures from protein data bank (PDB ID: 2EVQ, ${ }^{93} 1 \mathrm{UAO}, \underline{94} 1 \mathrm{YYB}^{95}$ ) are solvated in TIP3P $\mathrm{P}^{64}$ water and neutralized with sodium or chloride ions with the help of LEAP module from AMBER10 ${ }^{96}$ simulation package. AMBER99SB parameter set $\frac{63}{63}$ is used to describe peptides. Every box edge is at least $1 \mathrm{~nm}$ far from any protein atom.

Then energy minimization is carried out in two steps: 1, minimizing water and neutralizing ions only and, 2, minimizing the whole box. Then the system is heated gradually to simulation temperature in 100 ps with system volume kept 
Table 3.1: Summary of MD simulations carried out to test PHB and SFC models.

\begin{tabular}{|c|c|c|c|c|}
\hline Protein & $\begin{array}{l}\text { Starting } \\
\text { Structure }\end{array}$ & $\begin{array}{c}\text { Temperature } \\
(\mathrm{K})\end{array}$ & $\begin{array}{l}\text { Charge } \\
\text { Model }\end{array}$ & Trajectory length \\
\hline \multirow{6}{*}{ HP7 } & \multirow{6}{*}{1} & \multirow{3}{*}{310} & AMBER & $10 \times 20 \mathrm{~ns}$ \\
\hline & & & PHB & $20 \times 20 \mathrm{~ns}$ \\
\hline & & & $\mathrm{SFC}$ & $20 \times 20 \mathrm{~ns}$ \\
\hline & & \multirow{3}{*}{340} & AMBER & $5 \times 20 \mathrm{~ns}$ \\
\hline & & & PHB & $5 \times 20 \mathrm{~ns}$ \\
\hline & & & $\mathrm{SFC}$ & $5 \times 20 \mathrm{~ns}$ \\
\hline \multirow{3}{*}{ Chignolin } & \multirow{3}{*}{1} & \multirow{3}{*}{310} & AMBER & $10 \times 20 \mathrm{~ns}$ \\
\hline & & & PHB & $20 \times 20 \mathrm{~ns}$ \\
\hline & & & $\mathrm{SFC}$ & $20 \times 20 \mathrm{~ns}$ \\
\hline \multirow{11}{*}{ PDCD5-(1-26) } & 1 & 298 & AMBER & $10 \times 20 \mathrm{~ns}$ \\
\hline & \multirow{2}{*}{1} & \multirow{2}{*}{298} & PHB & $5 \times 20 \mathrm{~ns}$ \\
\hline & & & $\mathrm{SFC}$ & $5 \times 20 \mathrm{~ns}$ \\
\hline & \multirow{2}{*}{2} & \multirow{2}{*}{298} & PHB & $5 \times 20 \mathrm{~ns}$ \\
\hline & & & $\mathrm{SFC}$ & $5 \times 20 \mathrm{~ns}$ \\
\hline & \multirow{2}{*}{3} & \multirow{2}{*}{298} & PHB & $5 \times 20 \mathrm{~ns}$ \\
\hline & & & $\mathrm{SFC}$ & $5 \times 20 \mathrm{~ns}$ \\
\hline & \multirow{2}{*}{4} & \multirow{2}{*}{298} & PHB & $5 \times 20 \mathrm{~ns}$ \\
\hline & & & $\mathrm{SFC}$ & $5 \times 20 \mathrm{~ns}$ \\
\hline & \multirow{2}{*}{5} & \multirow{2}{*}{298} & PHB & $5 \times 20 \mathrm{~ns}$ \\
\hline & & & $\mathrm{SFC}$ & $5 \times 20 \mathrm{~ns}$ \\
\hline
\end{tabular}


constant. In minimization, all protein atoms are restrained with a force constant of $1000 \mathrm{kCal} / \mathrm{mol} \cdot \AA^{2}$. After the target temperature is achieved, the restraint is removed when performing a 100ps constant volume, constant temperature equilibration phase. In the next step, the restraint is applied again when the pressure is maintained at 1 bar with isotropic scaling for 500 ps. Then, another 500 ps constant pressure simulation is carried out before achieving equilibrium. At last, the production simulation is done under constant pressure and constant temperature condition. Multiple $20 \mathrm{~ns}$ long trajectories of each peptide are produced for analysis and comparison. SHAKE algorithm ${ }^{\frac{97}{1}}$ is utilized to reduce degree of freedom of bonds involving hydrogen atom in all simulations. Simple leap-frog integrator ${ }^{16}$ is used to propagate the simulation with a collision frequency of $3 \mathrm{ps}^{-1}$. Particle mesh ewald (PME) method ${ }^{71 / 72}$ is adopted to treat electrostatic interaction with a $1 \mathrm{~nm}$ real space-reciprocal space cutoff. Coulomb interaction is calculated with a simple 1 nm cutoff scheme.

Charge variation is only applied to the production phase. According to their published papers, the updating frequencies of PHB and SFC are different. In PHB simulations, backbone charges are checked and updated every 250 ps, while in SFC simulations, charges are updated every 100 fs. Both models require some in-house modification to the SANDER module of AMBER10 program package. All modifications are done in the same way described by Gao et.al. ${ }^{27}$ and Ji et.al., $\underline{92}$ and tested with the system in their respective paper.

\subsubsection{Trajectory analysis}

The trajectories are analyzed with several structural indicators. Here is the definition and usage of these indicators.

$R$ parameter : The " $\mathrm{R}$ parameter" 98 is a robust parameter for identifying structural variations of $\beta$-hairpin. It is defined as

$$
R=\sum_{i=1}^{n} \frac{R_{i}^{0}}{R i}
$$


where $R_{i}^{0}$ is the $i$ th $C_{\alpha}-C_{\alpha}$ distance in the NMR structure which is containing $n$ pairs residues in the $\beta$-hairpin. $R_{i}$ is the same distance in MD simulation. A value of $R \approx n$ indicates hairpin structure similar to NMR structure. In our case, $R=3$ for HP7 and $R=2$ for chignolin indicate their native (NMR) structure.

$J$-coupling : The J-coupling constant gives useful dihedral constraint to NMR structure determination of biological macromolecules. The translation between ${ }^{3} \mathrm{~J}$ constant and dihedral angle $\theta$ relies on the widely used empirical Karplus relation: $\underline{99}$

$$
{ }^{3} J(\theta)=A \cos ^{2} \theta+B \cos \theta+C
$$

where $A, B$ and $C$ are empirical parameters. In our study, the parameters from Pérez et.al. .100 are adopted.

\subsection{Results}

To judge the performance of both PHB and SFC models, unbiased MD simulations have been performed with three peptides representing different protein secondary structures. Multiple trajectories are obtained for every peptide in order to improve sampling efficiency. In what follows, the results are interpreted in both thermodynamic and structural perspectives. Comparisons are done for each peptide as well.

\subsubsection{HP7 and chignolin: $\beta$-hairpin}

$\beta$-sheet is one of the fundamental protein secondary structures. Numerous studies $^{93[94 / 101-112}$ have been done on the folding mechanism of $\beta$-hairpins, hoping to get a glimpse of protein folding mechanism. Much information about $\beta$-sheet folding is acquired by studying engineered $\beta$-hairpin. ${ }^{93} \mathrm{HP} 7$ is one of such engineered $\beta$ hairpin that helps to elucidate the folding of $\beta$-sheet. It consists of a 6 -residue loop and two 3-residue long $\beta$-strands (sequence KTW-NPATGK-WTE). Beside interstrand Hbonds, side chain interactions, including the interaction between aromatic 
side chains of W3 and W10, the cation- $\pi$ interaction originated from side chains of $\mathrm{K} 1$ and W10, are deemed important to form and stabilize this $\beta$-hairpin. Designed by Honda et.al., ${ }^{94}$ chignolin is another well-known and extensively studied $\beta$-hairpin. With only the length of 10 amino acid residues, it folds to a stable $\beta$ hairpin structure. Residues TYR2 and TRP9 forms a hydrophobic core stabilizing its native structure.

To start with, we present structural characteristics of HP7 and chignolin under all models. We've acquired simulation trajectories of HP7 under two temperatures, $310 \mathrm{~K}$ and $340 \mathrm{~K}$, one of which is close to the melting temperature of HP7 $\left(\sim 66^{\circ} \mathrm{C}\right)$, while all simulation of chignolin is conducted under $310 \mathrm{~K}$, close to its melting temperature of $312 \mathrm{~K}$. In $310 \mathrm{~K}$ trajectories, HP7 stayed in its native state exclusively, as can be seen from the distribution of all-atom root-mean square deviation (RMSD) relative to NMR structure and the free energy landscape shown in Figure 3.1 and 3.2 .

As shown in Fig 3.1, while AMBER and PHB models give almost the same probability density for both $\beta$-hairpins, SFC model exhibits an apparent RMSD shift to higher value. The mean value of RMSD from SFC model is 0.2-0.3 larger than both AMBER and PHB results. In simulations of chignolin, SFC gives a mean RMSD even higher $(\sim 0.4 \AA)$ than simulations of HP7. This indicates similar performance on overall structure by AMBER and PHB. It also implies lower stability of both hairpins under SFC model. As expected, larger structural variation is observed with by HP7 in $340 \mathrm{~K}$ trajectories.

To compare performance of different models, free energy landscape was plotted with trajectories of HP7 at $340 \mathrm{~K}$ and chignolin at $310 \mathrm{~K}$ (Fig 3.2). When examining the free energy landscape, the native state basin can be easily identified. Overall shape differences can be seen from the free energy landscape. In HP7 simulations, the non-native states have both lower $\mathrm{R}$ parameter and higher all-atom RMSD with AMBER force field. But with PHB or SFC model, the most populated non-native state has almost the same $\mathrm{R}$ parameter and higher all-atom RMSD than the native state. Since R parameter is describing backbone structure, 


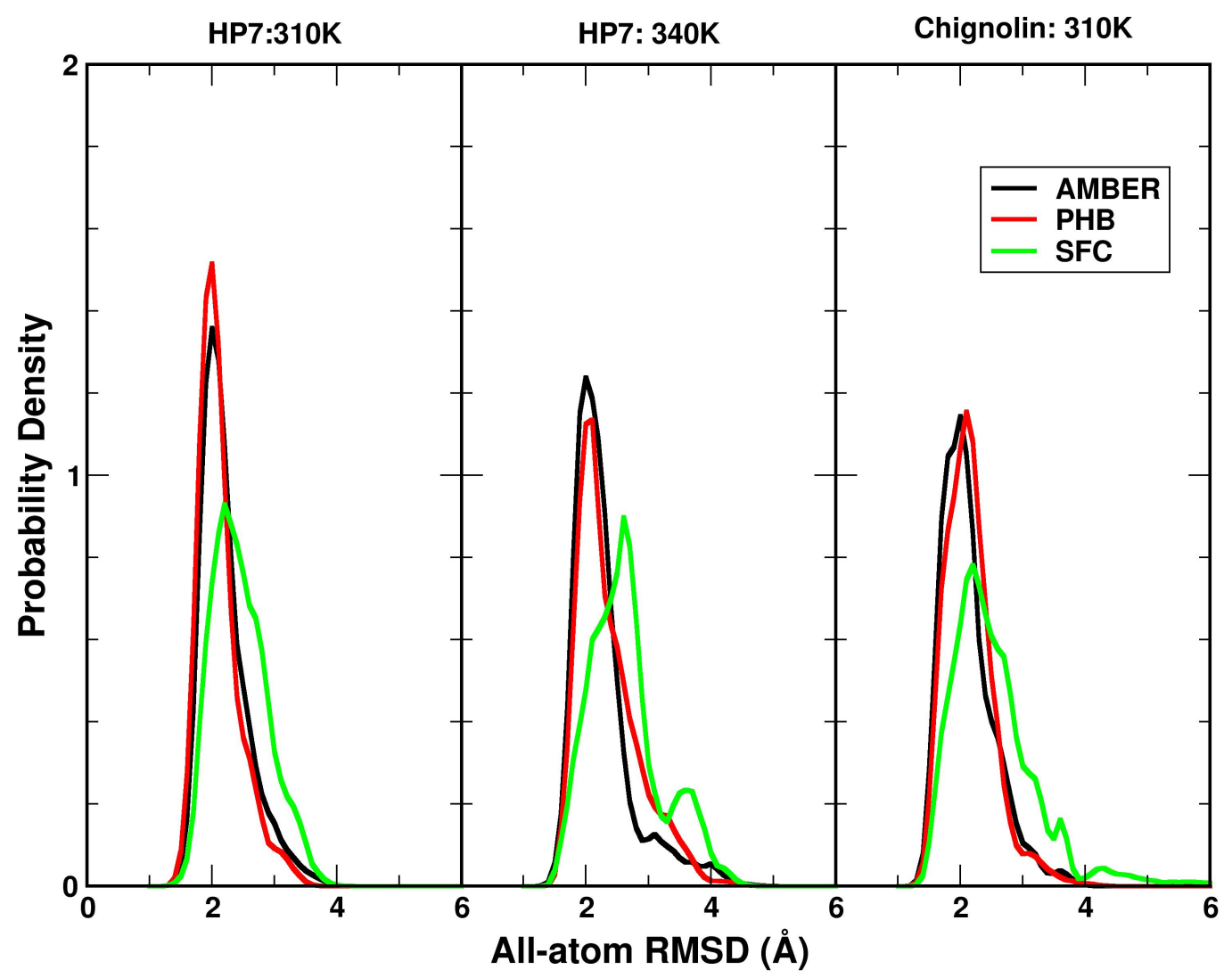

Figure 3.1: Distribution of all-atom root mean square deviation of $\beta$-hairpins. All-atom RMSD distributions for both HP7 and chignolin under all models are plotted. 

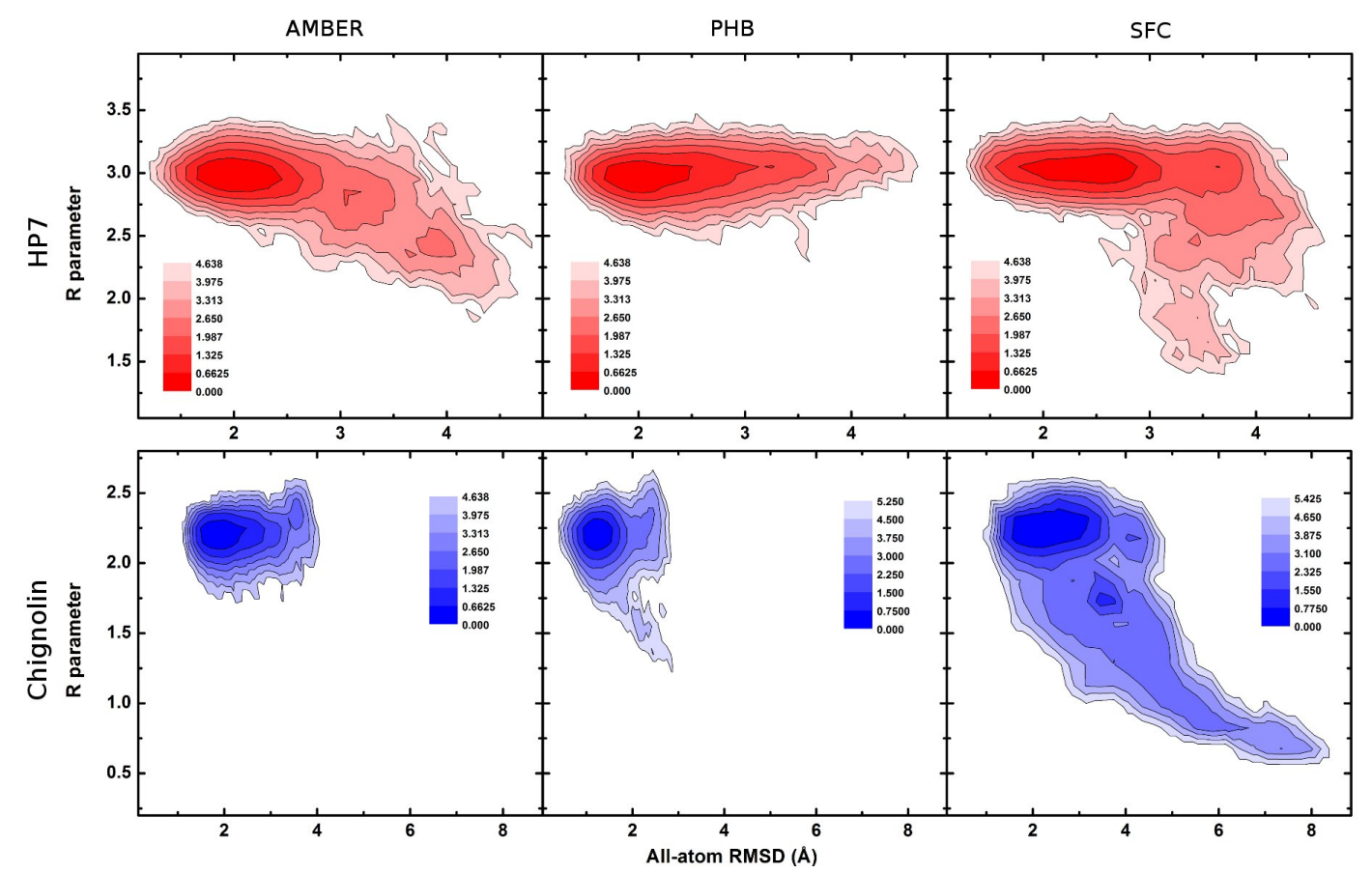

Figure 3.2: Free energy landscape of HP7 at $340 \mathrm{~K}$ and chignolin at $310 \mathrm{~K}$, generated with R parameter and all-atom RMSD. See text for detailed interpretation.

it is indicating stabilized backbone by both PHB and SFC model. This effect is less significant in chignolin simulations, perhaps because of the shorter $\beta$-strands in chignolin.

To test how well these three models hold the $\beta$-hairpin secondary structure, i.e. backbone conformation, dictionary of protein secondary structure (DSSP) algorithm is used to assign secondary structure to residues. Then the fraction of time that each residue resides in its native secondary structure is found, assuming residues THR2, TRP3, ASN4, LYS9, TRP10, THR11 of HP7 and TYR2, ASN3, THR8, TRP9 of chignolin in $\beta$-strand conformation, and loop -PATG- of HP7 and loop -PETG- of chignolin in $\beta$-turn conformation. The result is shown in Fig.3.3.

In simulations of HP7 under 310K, PHB model presents very similar results as AMBER force field. SFC model makes most residues of HP7 less stable in its native conformation, with both lower average value and larger deviation of fraction of time in native. However, the fraction of time in native for residues W3 and W10 remains 


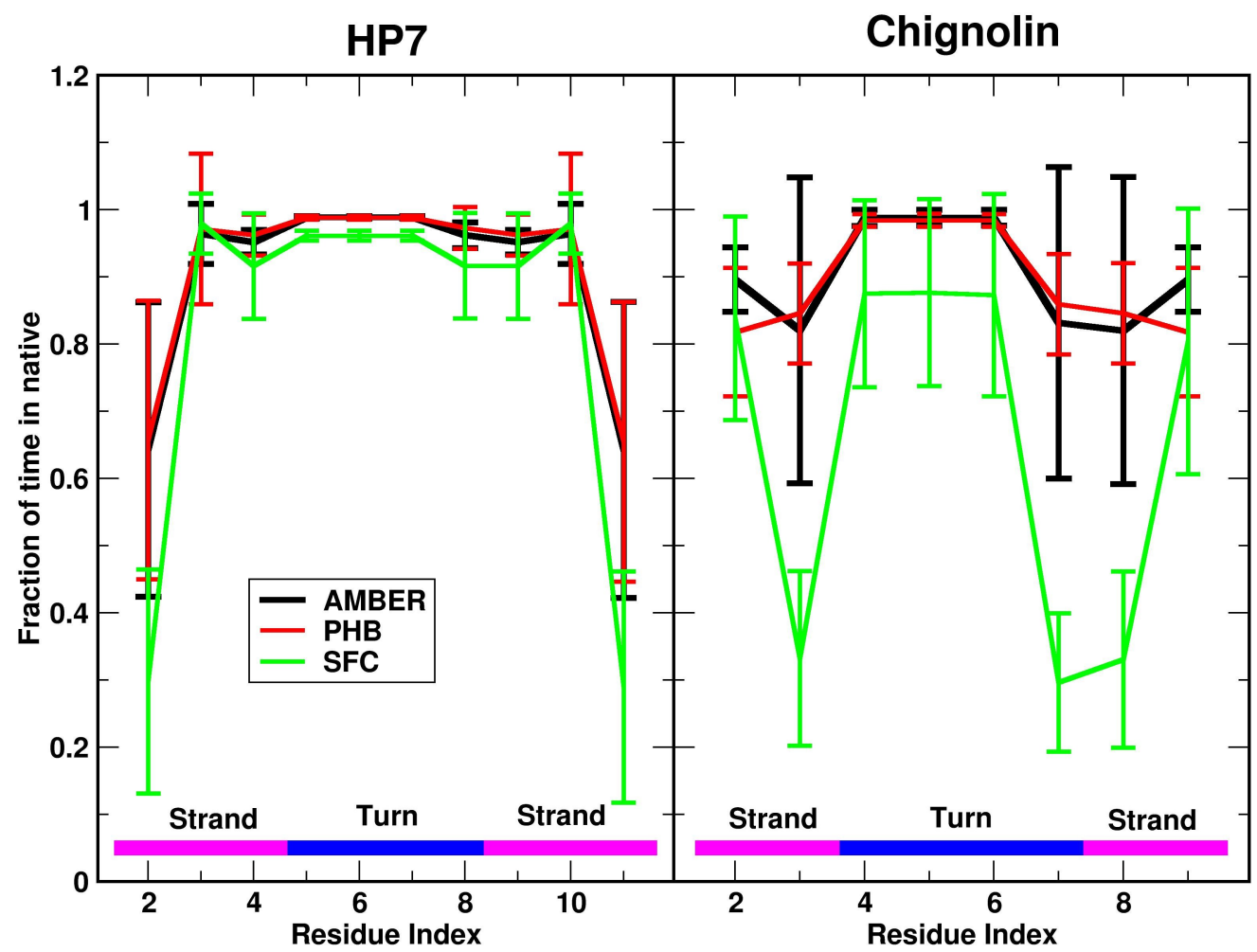

Figure 3.3: Fraction of time in native structure in simulations of $\beta$-hairpin. Native structure of residues is assigned according to NMR structure, as shown at the bottom of each figure. The mean value and standard deviation are calculated from all valid trajectories at temperature $310 \mathrm{~K}$. 
steady across all three models. This can be interpreted as the stabilizing effect from W3-W10 side chain interaction, which will be discussed later. Much more volatility is seen in chignolin simulations than HP7. Since the temperature of simulations, $310 \mathrm{~K}$, is very close to its melting temperature, more volatile structural dynamics is expected. The large standard deviation of fraction of time in native of chignolin can be interpreted as indicating such kind of dynamics. Specifically, AMBER presents less stable $\beta$-conformation for residues ASN3 and THR8 of chignolin than TYR2 and TRP9. This could be attributed to side chain interaction between TYR2 and TRP9. Further, SFC model gives the least stable structure for all residues among all three tested models. Residues ASN3, GLY7 and THR8 are only at their native conformation $\sim 30 \%$ of the time. However, the fraction of time in native from PHB model is very close to AMBER simulations, but with lower deviation, indicating stabilization of chignolin backbone.

Backbone hydrogen bond is further checked. Both Hbond occupancy and average Hbond length are plotted in Fig.3.4. It turns out that PHB model again presents closer result to AMBER than SFC model. In HP7 simulations, PHB results run side by side with AMBER data, yet with slightly lower backbone hydrogen bond occupancy, in general. For the first two of four backbone hydrogen bond in HP7, i.e. T2@O - T11@HN and K9@O - N4@HN, shorter hydrogen bond length is seen in SFC simulations, while Hbond length for the other two Hbonds are the same as AMBER and PHB model. Higher occupancy of these two hydrogen bonds is also observed under SFC model. The same lower hydrogen bond length is also observed in chignolin with SFC model. All three backbone Hbond length of chignolin is shorter under SFC model. However, the occupancy of the last two Hbonds, T8@O - D3@HN and G1@O - G10@HN, is the lowest among all three models. Short Hbond length points to a stronger representation of backbone Hbond by SFC model. Further, backbone Hbond interaction energy is calculated as following:

$$
E_{H b o n d}=\frac{q_{N} q_{C}}{r_{N C}}+\frac{q_{N} q_{O}}{r_{N O}}+\frac{q_{H} q_{C}}{r_{H C}}+\frac{q_{H} q_{O}}{r_{H O}}
$$



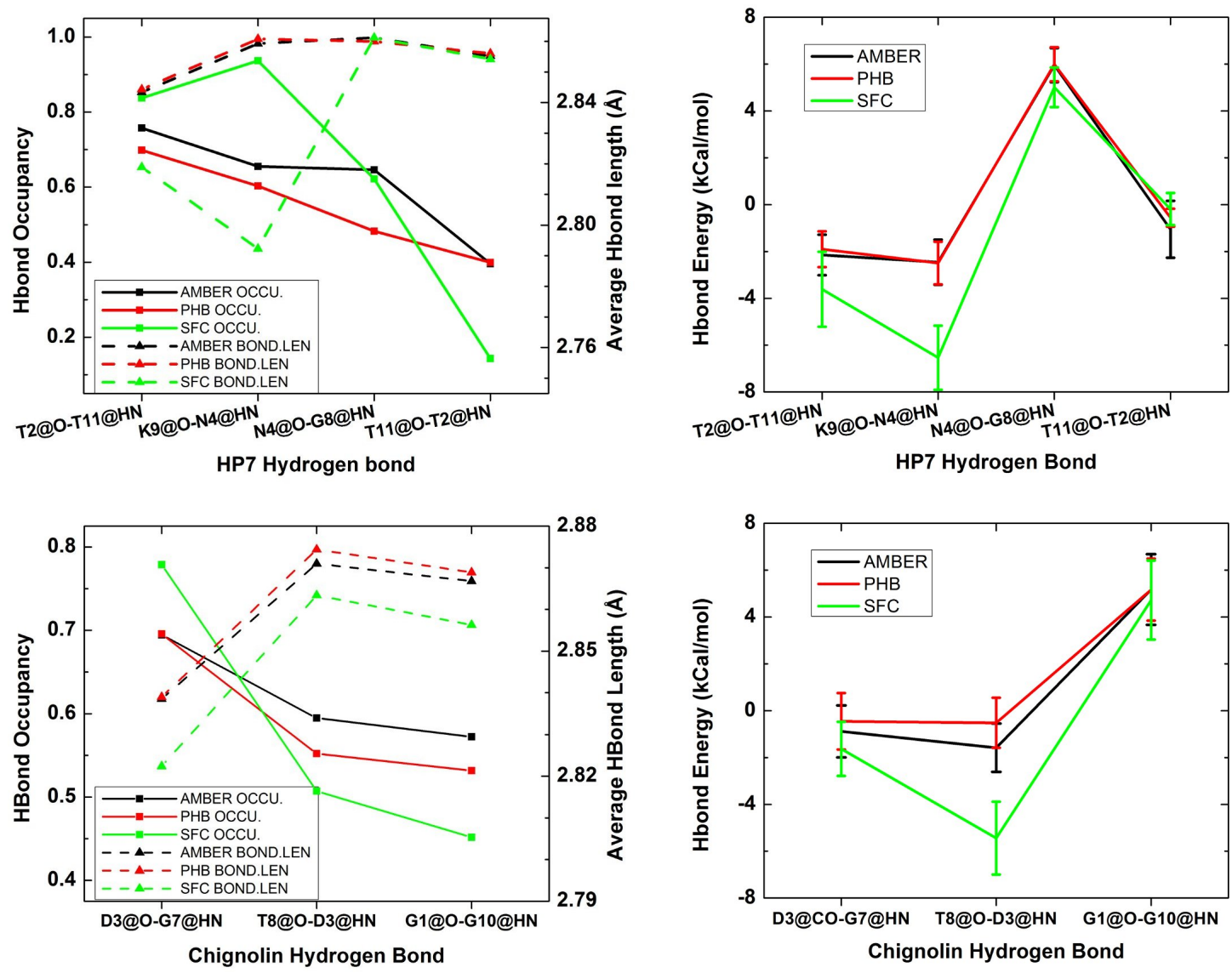

Figure 3.4: Backbone hydrogen bond occupancy and energy in $\beta$-hairpin simulations at $310 \mathrm{~K}$. 
As can be seen in Fig 3.4, the interaction energy given by SFC is lower for the first two Hbonds of HP7 and all three Hbonds in chignolin, comparing to AMBER or PHB. One of the characters that SFC model possesses is site-specific hydrogen bond strength. In our $\beta$-hairpin tests, there is much variance in hydrogen bond strength in SFC model comparing to AMBER. But the overall effect is to strengthen backbone Hbond in SFC model. Put together the hydrogen bond data and the $\mathrm{R}$ parameter presented in Fig 3.2 , we can see that both PHB and SFC model stabilize backbone structure of $\beta$-hairpins. It is expected since there is extra polarization energy introduced by these two models. To understand the low stability by SFC model, we have to turn our attention to side chain interactions.

Performance on side chain dynamics is examined with J-coupling analysis and side chain-side chain distance over all trajectories. It is reported side chain-side chain hydrophobic interaction is important to $\beta$-hairpin maintaining its native state. Site mutations have shown the importance of side chain-side chain interaction, and it's been used in engineering $\beta$-hairpins. Here, we analyse the J-coupling value about CA-CB bond ( $J_{\alpha \beta 2}$ and $J_{\alpha \beta 3}$ of aromatic side chains of both HP7 and chignolin. J-coupling values are calculated and plotted in Fig 3.5 versus frame number to make use of all trajectories. On the other hand, we simply monitor the distance between centres of mass of aromatic side chains in HP7 and chignolin to illustrate side chain-side chain contact. The distance data are also plotted in Fig 3.5 .

Though the J-coupling plot is not a time series, and values for residue Y2 of chignolin deviate from experimental values, transition amongst all rotamers are identifiable. There are more trans-gauche transitions in SFC simulations and less in PHB simulations for both W3 and W10 of HP7. When the simulation length is considered, the numbers of trans-gauche transitions for W3 in both AMBER and SFC are the same, larger than PHB simulations. In particular, there's no transition observed for W10 in PHB trajectories. Further, the fluctuation magnitude of W3W10 side chain distance is in the same order for these three models. All these data show that PHB model tends to strengthen W3-W10 side chain interaction 

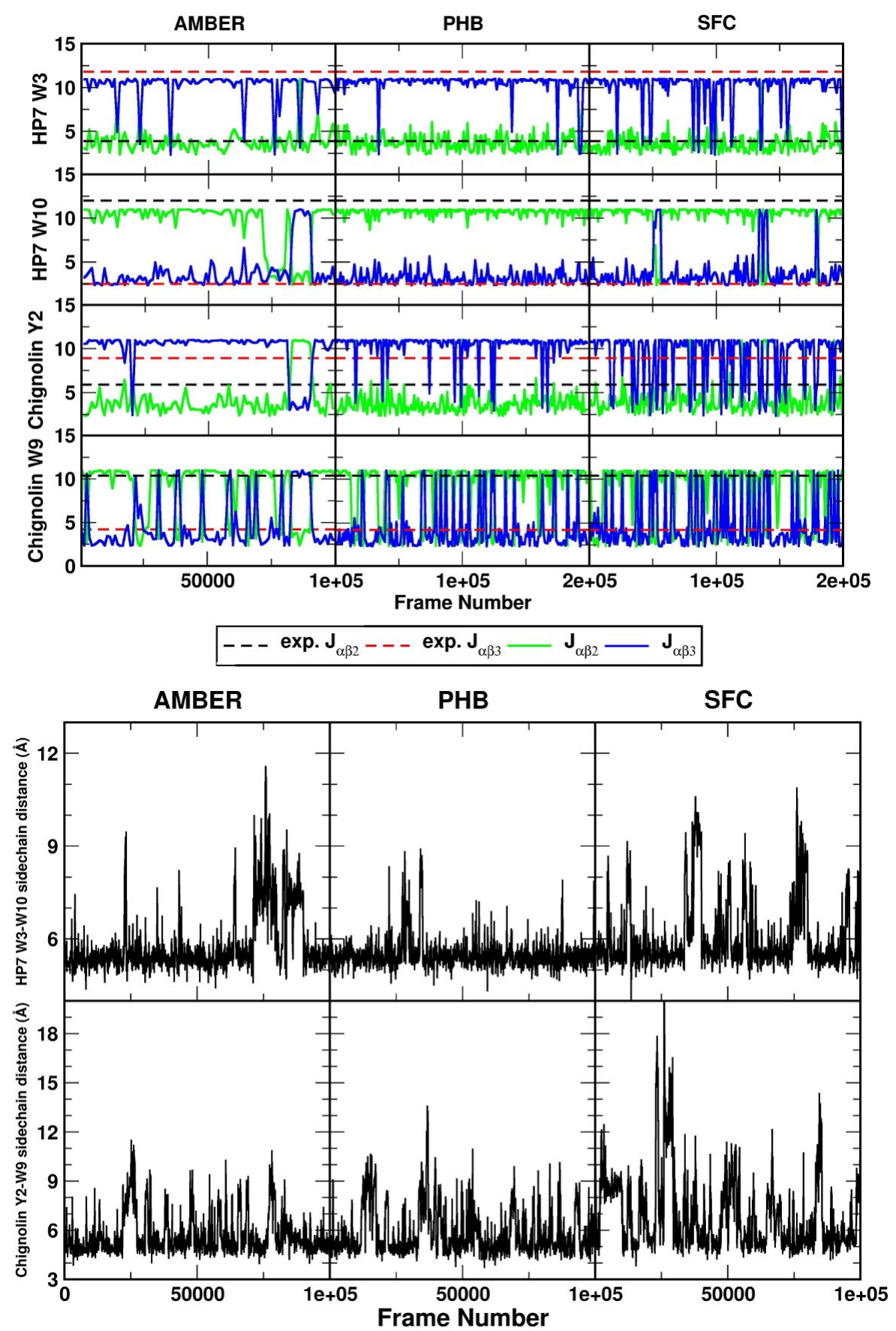

Figure 3.5: J-coupling and aromatic side chains distance in simulations of $\beta$ hairpins. Upper figure: J-coupling factor of $\beta$-hairpin aromatic side chains. Lower figure: Side chain aromatic ring centre of mass distance as a function of frame number for hairpins. 


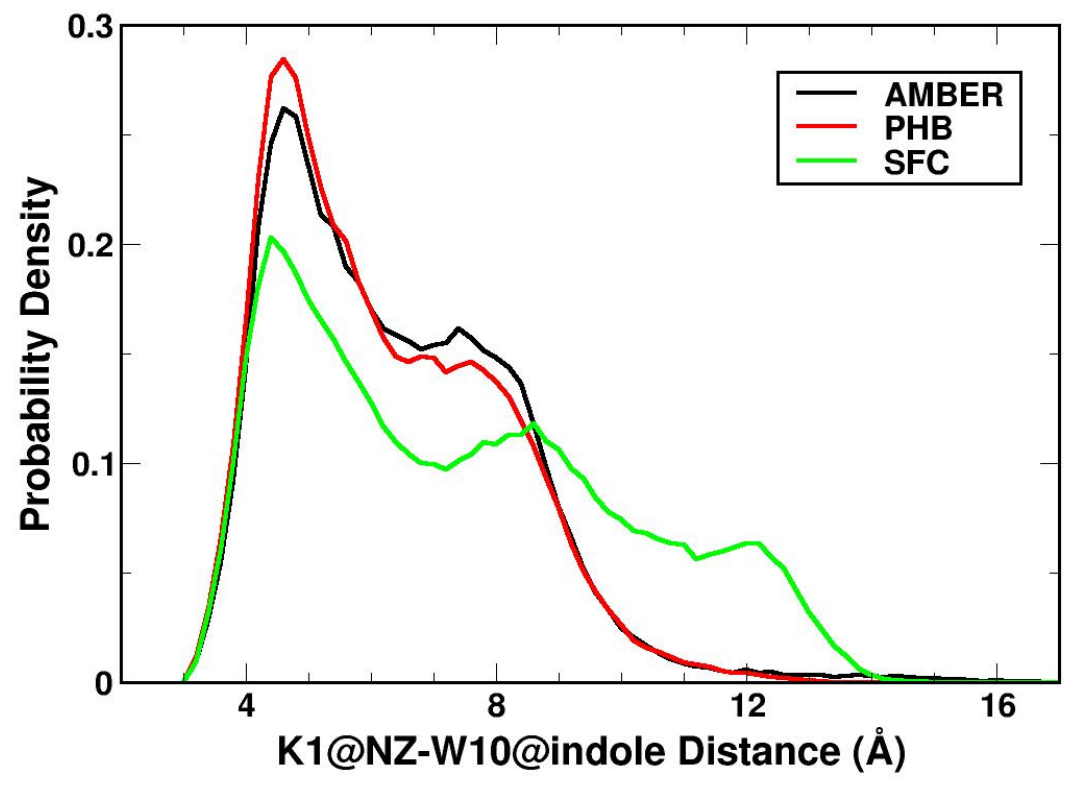

Figure 3.6: HP7 K1-W10 side chain distance distribution characterizing the cation$\pi$ interaction. Distribution of distance between K1@NZ and centre of mass of W10 indole ring is plotted.

at the native state of HP7, and SFC model tends to weaken the interaction. On the other hand, in chignolin simulations, PHB model presents higher trans-gauche transition number for Y2 than AMBER, and almost the same for W9, while SFC model presents higher transition numbers for both residues. The distance between side chains of Y2 and W9 is in the order of AMBER; PHB ; SFC. The side chain interaction is represented weakest by SFC model, resulting more unfolding events observed.

Next, we examine the cation- $\pi$ interaction between residues K1 and W10 of HP7, by looking at the distribution of distance between K1@NZ atom and the centre of mass of W10 indole ring. As shown in Fig 3.6, the probability distribution of this distance is very similar in AMBER and PHB trajectories, with a little accentuating by PHB model, while a shift to longer distance is noticed in SFC simulations, indicating SFC model weakens the K1-W10 side chain cation- $\pi$ 
interaction. It is reported this interaction is important for maintaining HP7 native state, hence this could be a contributing factor to the lower stability of HP7 discussed earlier.

To sum up, both PHB and SFC model have stabilizing effect on $\beta$-hairpin backbone hydrogen bonds over AMBER. As a result, the lengths of backbone hydrogen bonds are shortened. Similar or stabilized dynamics is seen with PHB model. However, the side chain-side chain interactions, both aromatic interaction and cation- $\pi$ interaction, are not well represented by SFC model. This could be the cause of the less stability in simulations under its regulation.

\subsubsection{PDCD5-(1-26): $\alpha$-helix}

PDCD5-(1-26) $)^{95}$ is the N-terminal 26-residue fragment of human programmed cell death 5 (PDCD5) protein. It is of importance as involved in the regulation of cell apoptosis. The structure of PDCD5-(1-26) has been determined with circular dichroism (CD) and nuclear magnetic resonance (NMR) methods. It turns out to be a stable $\alpha$-helix spanning from residue D3 to A19 in water solution.

We have examined structural characteristics of PDCD5-(1-26) under the regulation of AMBER99SB parameter and two charge variation models. It is estimated from far-UV CD spectrum that PDCD5-(1-26) contains $~ 54.4 \%$ of $\alpha$-helix (yellow vertical line in Fig 3.7). In our simulations, the average helical contents under AMBER99SB, PHB and SFC are 58.70\%, 59.36\% and $63.29 \%$ respectively. As shown in Fig 3.7, the distribution of helical content under SFC model is mostly between $60 \%$ and $80 \%$, as opposed to the other two models, whose distribution of helical contents are mainly between $50 \%$ to $70 \%$. When comparing helical content from AMBER99SB and PHB simulations, we can claim that AMBER99SB result is closer to experimental value. PHB result have higher estimation of helical content than AMBER99SB. Specifically, as can be seen in Fig. 3.8, residue 2-6 spend more time in $\alpha$-helical conformation with SFC model applied. AMBER99SB and PHB present similar data for this segment of PDCD5-(1-26). But for the other end of the helix, residue 17-21, PHB and SFC models give similar probability in 


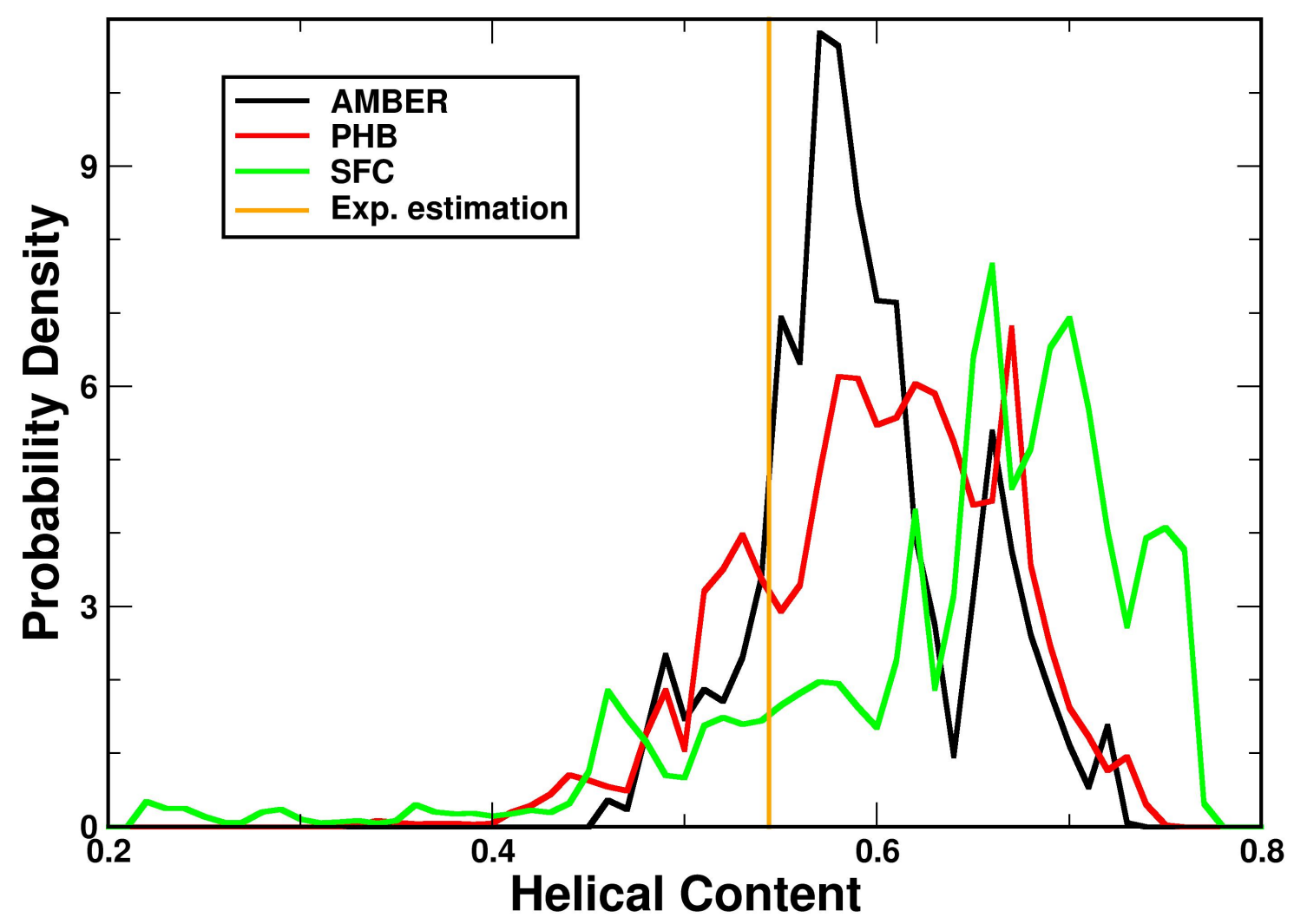

Figure 3.7: Helical content distribution in PDCD5-(1-26) simulations. Experimental estimation of helical content of PDCD5-(1-26) is drawn as a vertical line (yellow). 


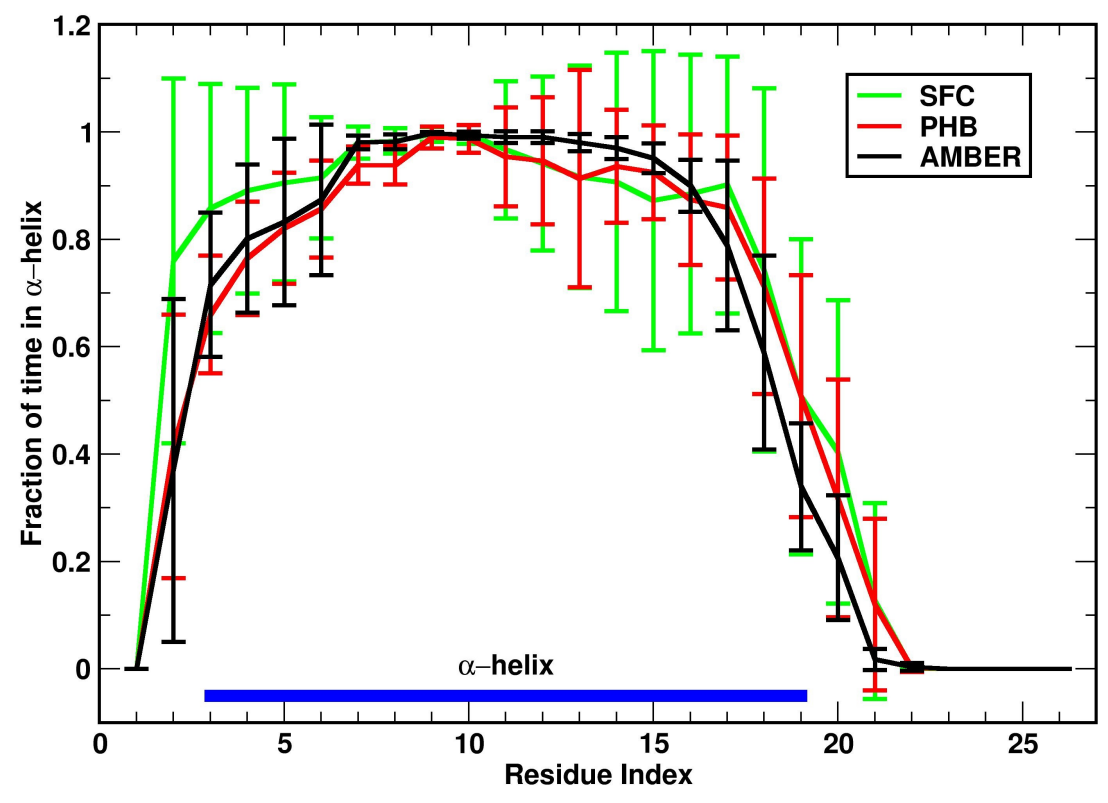

Figure 3.8: Fraction of time in $\alpha$-helical conformation per residue for PDCD-(126 ). The native $\alpha$-helix is denoted at the bottom.

$\alpha$-helical conformation, higher than AMBER99SB. It is worth mentioning that for most residues within $\alpha$-helix region, charge variation results in higher variation in fraction of time in $\alpha$-helix conformation. After all, system Hamiltonian is tempered constantly during charge variations.

Since $\alpha$-helix is the main secondary structure here, all $i+4 \rightarrow i$ hydrogen bonds are checked after simulation. Hydrogen bond occupancy and length are presented in Fig. 3.9 statistically, in which $i$ was along $x$ axis. Similar to simulations of $\beta$ hairpins, occupancy of most hydrogen bonds has higher values under SFC model than the other two models. Comparable occupancy and hydrogen bond length can only be seen for 4 hydrogen bonds, namely LEU6@O-ARG10@NH, LEU9@OARG13@NH, GLN12@O-GLU16@NH and GLU16@O-LYS20@NH. Lengths of these 4 hydrogen bonds are very close as well, while lengths of other hydrogen bonds are significantly shorter than hydrogen bond lengths from the other two models. 

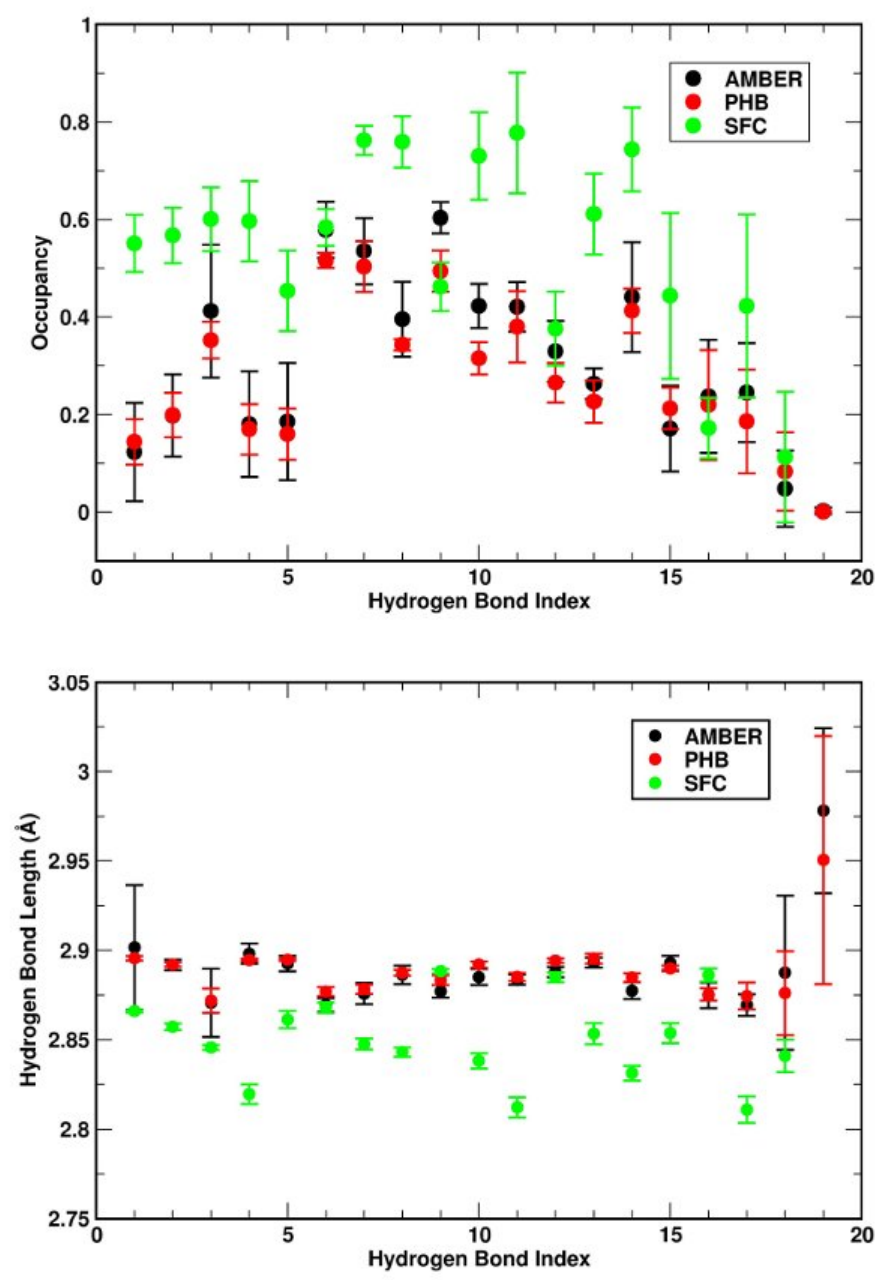

Figure 3.9: Statistics of $i+4 \rightarrow i$ hydrogen bond in PDCD-(1-26) occupancy and length.

These data indicate stronger hydrogen bonds in SFC model, resulting in higher average fraction of time in $\alpha$-helix for some residues and higher helical content discussed before.

On the other hand, PHB and AMBER99SB model present similar occupancy and length for all $i+4 \rightarrow i$ hydrogen bonds. However, lower standard deviation of occupancy and hydrogen bond length can be observed with PHB model than AMBER99SB. This is due to the polarization energy introduce by PHB model. With the polarized partial atomic charge added, both PHB and SFC model stabi- 

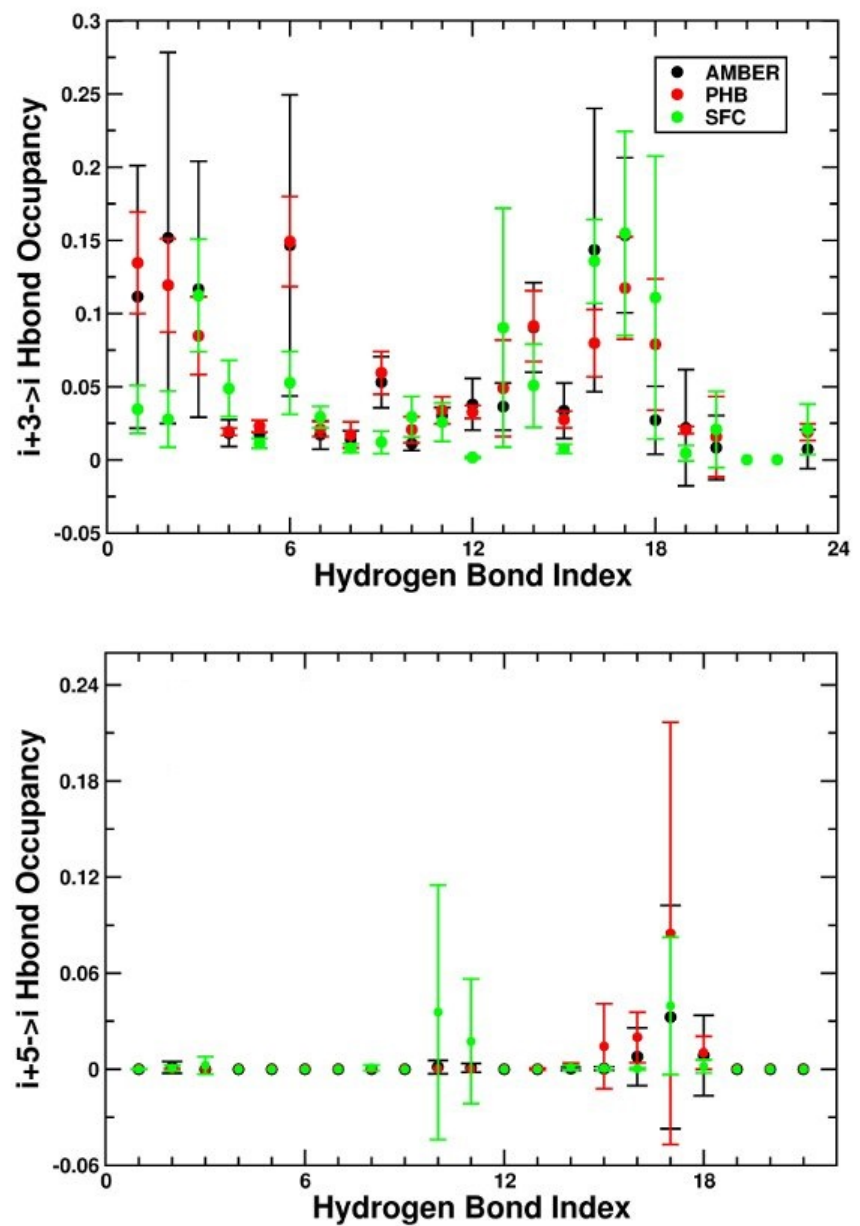

Figure 3.10: Occupancy of $i+3 \rightarrow i$ and $i+5 \rightarrow i$ backbone hydrogen bonds in PDCD-(1-26) simulations.

lize this $\alpha$-helix by strengthening backbone hydrogen bonds. But SFC model has more significant effect than PHB model, hence higher helical content is observed. The SFC model strengthens hydrogen bonds so much that the occupancy of some hydrogen bonds doubled over AMBER simulations. Hydrogen bond lengths are shortened significantly under SFC model. To sum up, both PHB and SFC model strengthen backbone hydrogen bonds of $\operatorname{PDCD}(1-26)$, hence stabilize its structure.

Meanwhile, both $i+3 \rightarrow i$ and $i+5 \rightarrow i$ backbone hydrogen bonds are examined to illustrate the possibility of transferring from $\alpha$-helix to other helix format, i.e. $3{ }_{10}$-helix and $\pi$-helix. All data are plotted in Fig.3.10. Similar to data we presented 
before, PHB model have a more similar behaviour with AMBER99SB model than SFC model. Generally, $i+3 \rightarrow i$ hydrogen bonds have higher occupancies than $i+5 \rightarrow i$ hydrogen bonds. For residues near the N-terminus, simulations under SFC model have lower $i+3 \rightarrow i$ hydrogen bond occupancy. At the other end of the helix, SFC model data have comparable or higher $i+3 \rightarrow i$ hydrogen bond occupancy than the other two models. This is resulted from the strengthened $i+4 \rightarrow i$ hydrogen bonds with SFC model. Backbone hydrogen bond forming groups spend more time in $i+4 \rightarrow i$ hydrogen bonds, other than other form. Lastly, there is only low possibility of forming $i+5 \rightarrow i$ hydrogen bond at the C-end of the helix due to the unstructured tail.

\subsection{Discussion}

Two backbone fluctuating charge models are utilized to simulate three small protein molecules. The results are compared with results from AMBER99SB force field parameter set. Without surprise, the two fluctuating charge models give strengthened backbone hydrogen bonds. Therefore, stabilized backbone is observed with these two models. However, the overall performance of both fluctuating charge models is not satisfactory. For instance, SFC model strengthen backbone interactions while weaken side chain interactions at the same time in simulations with $\beta$-hairpin. PHB model tend to over stabilize the backbone and $\pi-\pi$ interaction of HP7 sidechains.

The idea of varying backbone charge to incorporate polarization effect is naturally inconsistent with force field. Also, treating backbone and side chains of peptide separately is physically wrong. Therefore, the significance and usefulness of these models are severely limited.

The good side of the story is that these models are computationally inexpensive. The computation time consumption is only a little more than normal MD simulation. These models could be useful to some specific applications. 


\section{Chapter 4}

\section{Environment Response Polarized Protein Specific Charge}

In this chapter, polarized protein specific charge (PPC) is applied to Yersinia protein tyrosine phosphatase (PTPase), YopH, to study dynamics of WpD loop which governs the opening of substrate binding pocket of YopH. Periodically updating of PPC has been explored to improve accuracy in MD simulations with PPC applied. In this study, improvement has been proposed to advance efficiency of PPC-update by determining local electrostatic environment that residues reside in.

\subsection{Introduction}

To achieve higher accuracy and efficiency in all-atom MD simulation by including polarization, fluctuating atomic charge is a simple and intuitive approach. For instance, a set of more accurate atomic charge can be obtained via self-consistent

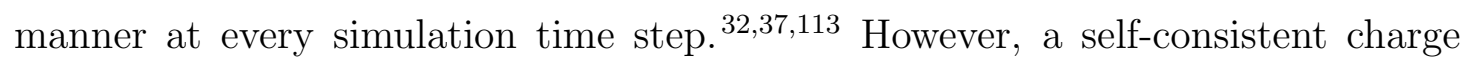
fluctuating model takes as much CPU time as polarizable force field with atomic dipole. It consumes about 4-5 times more computation time than conventional MD simulation. 37 On the other hand, polarizable force fields also give rise to better energy representation ${ }^{30}$ over simple atomic charge model. Such kind of force fields, e.g. AMOEBA,, 30 introduce new parameters, such as atomic dipole or quadrupole 
, to achieve more accurate energy functions. At the same time, more complexity is introduced as well, which makes the parameterization task more daunting than before. Furthermore, all self-consistent polarization methods, such as abovementioned charge response kernel or AMOEBA polarizable force field, have to utilize damping function of some form to prevent computation breaking down, known as polarization catastrophe. In this chapter, we explore the possibility of achieving better dynamical performance with fluctuating atomic charge, without self-consistent calculation of electrostatic energy, which consumes less computational time than polarizable force field or self-consistent fluctuating charge models at the same time.

Polarized protein-specific charge $(\mathrm{PPC})^{47}$ was first developed to model electrostatic interactions of protein molecules. It is made possible by fragmentation of protein molecule with molecular fragmentation with conjugated caps (MFCC) method. PPC is based on the rationale that every amino acid residue is under its own local environment, i.e. electrostatic field. The local environment alters, and also is altered by, the electronic structure of the amino acid residue, as well as charge distribution. Adopting a set of atomic partial charge that represents the electronic structure of a molecule under its local environment, other than a set of general atomic charge disregarding its environment, should give more accurate energy representations. Indeed, PPC has been successfully applied to many simulation studies ranging from calculating binding affinity to protein folding. With this rationale in mind, we can infer that pre-fitted PPC shall be well-behaved in protein native state simulations, where no drastic conformational change occurs. However, in other cases that involves more significant structural variation, such as protein folding, we cannot fit a set of PPC beforehand and use it throughout the whole folding, for instance. To overcome this shortcoming, the PPC-update scheme is proposed and examined in the past few years. For example, Xu et.al. ${ }^{114}$ first tried PPC-update scheme in a study of hydrogen-bond stability in MD simulation. The hydrogen-bonds were more stable with PPC other than AMBER amino acid specific charge. Due to the very high overhead of PPC-update, only short trajectories can be obtained. It may be sufficient in this specific case, since the structural 
variation is not very significant even though tens of hydrogen-bonds were broken and reformed during simulation. Another case study with PPC-update was conducted by Li et.al.. ${ }^{26}$ They applied PPC-update to the Zinc binding site of DFsc, a de novo designed dizinc metalloprotein, while keeping charges of other parts of the protein static. Very accurate zinc binding pocket structure was given by PPC-update, way better than structures AMBER parameters gave.

However, there are defects along with the PPC-update scheme. First of all, the computational expense is very high when PPC-update applied. To achieve convergence of atomic charge, several (usually 4-5) times of full quantum chemical calculation must be done. Even with a fragmentation method, the consumption of computation time makes it hard, or even impossible to achieve trajectories with decent length. If with a large system, it's even worse. On the other hand, aforementioned PPC-update studies didn't achieve very high efficiency. The updates are attempted only on fixed time points. There's no information about the molecule or local electrical environment whatsoever utilized while initiating the charge update. Even though both charge updating for whole protein and updating only part of protein are valid as proved by previous studies, no distinction between these two is made during the updating. These overlooks could result in wasting resources as it is possible that some updated residues may remain same or similar before and after updating. Therefore, further development of PPC-update was made to improve on these aspects. Adaptive hydrogen bond specific charge $(\mathrm{AHBC})^{1155}$ was proposed aiming at these points. The basic idea of AHBC is that, since hydrogen bonds are critical to protein structure and dynamics, we can use the formation and breaking of hydrogen bonds as the criterion to decide whether or not to perform charge update. Though there are some case studies with this method which give good results,,$^{26|115| 116}$ we have to say that the criterion used here is problematic. If we introduce polarization by updating PPC charge upon hydrogen bond formation, it is very likely that this hydrogen bond would have been strengthened upon formation. In other words, hydrogen bond is both the cause and result of our action of charge updating. Here in the last part of this 
thesis, I propose and implement a new charge updating simulation scheme with PPC, with which I hope the defects should be overcome.

Protein tyrosine phospatases (PTPase) comprise a large family of enzymes that, together with protein tyrosine kinases, regulate cellular protein phosphorylation level. It's critical to cellular signaling, hence is involved in various kinds of diseases, such as cancers and metabolic diseases. The catalysis mechanism had long been studied with various methods. In spite of the structural variations among different PTPases, the catalytic reaction depends on two loops about a substrate binding pocket. $\stackrel{117}{ }$ The first, namely phosphate bind loop or "P-loop", has a conserved sequence, $\operatorname{HCXXGXR}(\mathrm{S} / \mathrm{T})$, which includes a nucleophilic cystine. The other loop contains a conserved WpD sequence, hence called the WpD loop. It's a flexible binding loop. The common mechanism of PTPase catalyzed hydrolysis of phosphotyrosine $\sqrt{1181119}$ is briefly described as following. Upon substrate binding, the first conformational change occurs to the $\mathrm{WpD}$ loop. It transits from open to close conformation, bringing the essential ASP356 into the pocket. The conserved ASP356 is proposed to act as a general acid, providing a proton to the scissile oxygen of the substrate. Then the CYS403 on the P-loop serves as a nucleophile to accept the phosphoryl group dissociated from phosphotyrosine, forming a phosphocysteine intermediate. After the dissociation of the substrate leaving group, the side chain of GLN446 flips into the active site to stabilize a water molecule which is positioned along the S-P bond. The second chemical step involves the -OH group of THR410 interacting with the scissile sulfur of the intermediate. At last, the phosphoryl group dissociates from CYS403. This mechanism implies the flexibility of $\mathrm{WpD}$ loop plays an important role in the reaction process. As a matter of fact, various experiments verify that changing the flexibility of the $\mathrm{WpD}$ loop does affect the catalytic activity of PTPases. Increasing the flexibility by mutating residues in the $\mathrm{WpD}$ loop to alanine can boost up the catalytic activity up to 3-fold. Therefore, accurate description of $\mathrm{WpD}$ loop dynamics is an important premise to studying detailed catalytic mechanism of PTPase computationally.

The WpD loop has been examined with both spectroscopy and MD simulations. It is clear that the $\mathrm{WpD}$ loop exhibits rapid dynamics between open and 
close states while no substrate present. In substrate bound PTPase, the WpD loop remains in closed state. Juszczak et.al $\stackrel{120}{1}$ find out with UV resonance Raman spectroscopy that the energy barrier between the open and closed forms is very small. The equilibrium population of the two states is approximately equal. Further more, the rate constant of the rapid open-close transition WpD loop dynamics is about $2.6 \times 10^{8} s^{-1}$, which is $4 n s^{-1}$ in period. Computationally, Peters et.al. ${ }^{121}$ studied the human origin protein tyrosine phosphatase $1 \mathrm{~B}$ (PTP1B) in both ligand-bound and ligand-free states. Though the significance of their work is undermined by the short simulation time, useful information is presented about the dynamics of PTP1B, especially dynamics of the binding pocket. A more comprehensive MD study of PTPase is done by Hu et.al.. ${ }^{122}$ They have studied the bacterial YopH with both equilibrium MD simulation and locally enhanced sampling. The dynamical difference between wild type and mutant C403S binding pockets is demonstrated in their work. However, the definitions of the open and close states of $\mathrm{WpD}$ loop are questionable.

In this chapter, we study dynamics of YopH binding pocket with our new PPCupdate method. The main objectives that we're trying to achieve is 1) to try to model the dynamics of the $\mathrm{WpD}$ loop, 2)demonstrate the importance of including polarization into MD simulations even in processes that don't involve high energy barriers such as loop dynamics, 3)illustrate the importance of residue C403 in maintaining $\mathrm{WpD}$ loop dynamics in ligand-free state. 


\subsection{Method}

\subsubsection{General Considerations}

PPC is proposed to give rise to more accurate electrostatic energy for protein molecules. ${ }^{47}$ However, in real application, PPC fitted from similar structures usually varies much, i.e. PPC has structure dependency, which is not preferred in MD simulation. This inconvenience rises from the basic philosophy of PPC, which is the atomic charge should reflect the local environment. So, to be rigorous, PPC can only be fitted with the lowest energy structure, and is only valid in the vicinity of that structure in the whole conformation space. This will largely limit the applicability of PPC method. To alleviate the limitation, a simple measure can be adopted, which is, during a MD simulation, to replace current charge with newly fitted PPC based on new structure whenever the protein molecule moves away from the previous structure in the conformational space. This is the so-called PPC-update simulation scheme. It can be seen as an improvement to PPC when applied to MD simulation.

Improved performance is achieved with PPC-update scheme in several cases. $26 \mid 116$ The common procedure of PPC-update is that, at some time point which is chosen either arbitrarily or with some criterion, a set of PPC is generated before the MD simulation carries on with this set of newly generated charge. There are several differences among different implementations of the updating schemes, which lie in several aspects including choice of updating criterion and charge-fitting method to generate PPC. As discussed before, to fully exploit the potential of PPC, we are to perform charge updating only when the structure or the local environment deviates from before. In other words, a good criterion is one that defines the deviation reasonably and accurately. On the other hand, the charge-fitting method adopted must be robust to all possible states or structures of our molecule. It should minimize the structure dependency of PPC since PPC has a mean-field nature, and present charge distribution under local environment properly.

Since PPC-update is done at discrete time points along the simulation, there should be a proper minimal time between consecutive updatings. Varying charges 
to part of the simulation system is a perturbation to potential energy. Hence, a certain mount of time is required for the system to regain equilibrium. This minimal interval time depends on the properties of our studied system. It should be at least the minimal time the simulated system takes from a nonequilibrate state to equilibrium.

\subsubsection{Selection Of The Updating Criterion}

In a periodic all-atom representation of simulation systems with point charges, the electrostatic energy of the system can be written as

$$
E_{\text {elec }}=\frac{1}{2} \sum_{i} q_{i} \Phi_{i}
$$

where $q_{i}$ and $\Phi_{i}$ are atomic charge of atom $i$ and the electrostatic potential acting on atom $i$ respectively. Under such site-specific charge representation, electrostatic polarization can be interpreted as interplay between atomic charge and electrostatic potential. Usually, the electrostatic energy is obtained in a self-consistent manner, such as in charge response kernel model, i.e. iterating to obtain converged electrostatic energy. With PPC, a different approach is adopted. Residue based fragmentation is firstly conducted on peptide chains. Then ab initio charge fitting with RESP method ${ }^{49}$ is performed on each segment with conjugated caps, with charges of other atoms as background during ab initio calculation. Iteration is performed before reaching self-consistency. The electrostatic energy is obtained with the point charge generated at the end. Hence, self-consistency is promised before we calculate electrostatic energy with PPC.

As mentioned before, PPC has conformation dependency to some extent. To accommodate such self-consistent PPC in MD simulations, charge updating is needed to make sure that current charges give the best electrostatic energy corresponding to current conformation. As the polarization process can be seen as the interplay between atomic charge and electrostatic potential, I decide to use electrostatic potential as the indicator to initiate charge updating. For part of the 
system, for example, an amino acid residue within peptide chain, the electrostatic potential acting on this subsystem is resulted from all atoms that don't belong to this part. The electrostatic potential contains all polarization information from other part of the system. Furthermore, the electrostatic potential is easy to extract from MD simulations. Very little extra effort is needed to obtain the electrostatic potential, since all these values are actually used to calculate electrostatic energy at every MD step.

As discussed in the previous section, we need to identify different states by utilizing electrostatic potential. In other words, we have to identify different "environment" residues reside in so that the decision to perform charge updating can be made, i.e. residues respond to environment. Since electrostatic potential reflects all properties of the environment, I use the difference of electrostatic potential at two time points to determine whether the environment is changed. Various functions and algorithms can be utilized here to identify the difference. In this thesis, I just take the simplest measure, i.e. the root mean square (RMS) of electrostatic potential between two time points, to implement the charge updating scheme. The root mean square (RMS) of electrostatic potential acting on a set of $n$ atoms between two time points $t$ and $t-1$ is as follow.

$$
R M S_{\phi}=\sqrt{\frac{\sum_{i=1}^{n}\left(\phi_{i}^{t}-\phi_{i}^{t-1}\right)^{2}}{n}}
$$

Under equilibrium, not only the electrostatic energy of the whole system but also the electrostatic energy of a sub-system should have a constant mean value. Therefore, in a protein solution simulation with fixed atomic charge, the electrostatic potential acting on a specific residue gives rise to electrostatic energy with static mean. Here, the assumption is made that under equilibrium condition, the RMS of electrostatic potential acting on a specific amino acid reside obeys the gaussian distribution. Here the first step of our implementation is to check the electrostatic potential. If the potential RMS relative to previous check time is greater than a threshold value, the charge updating is performed, otherwise previous charge is preserved. 


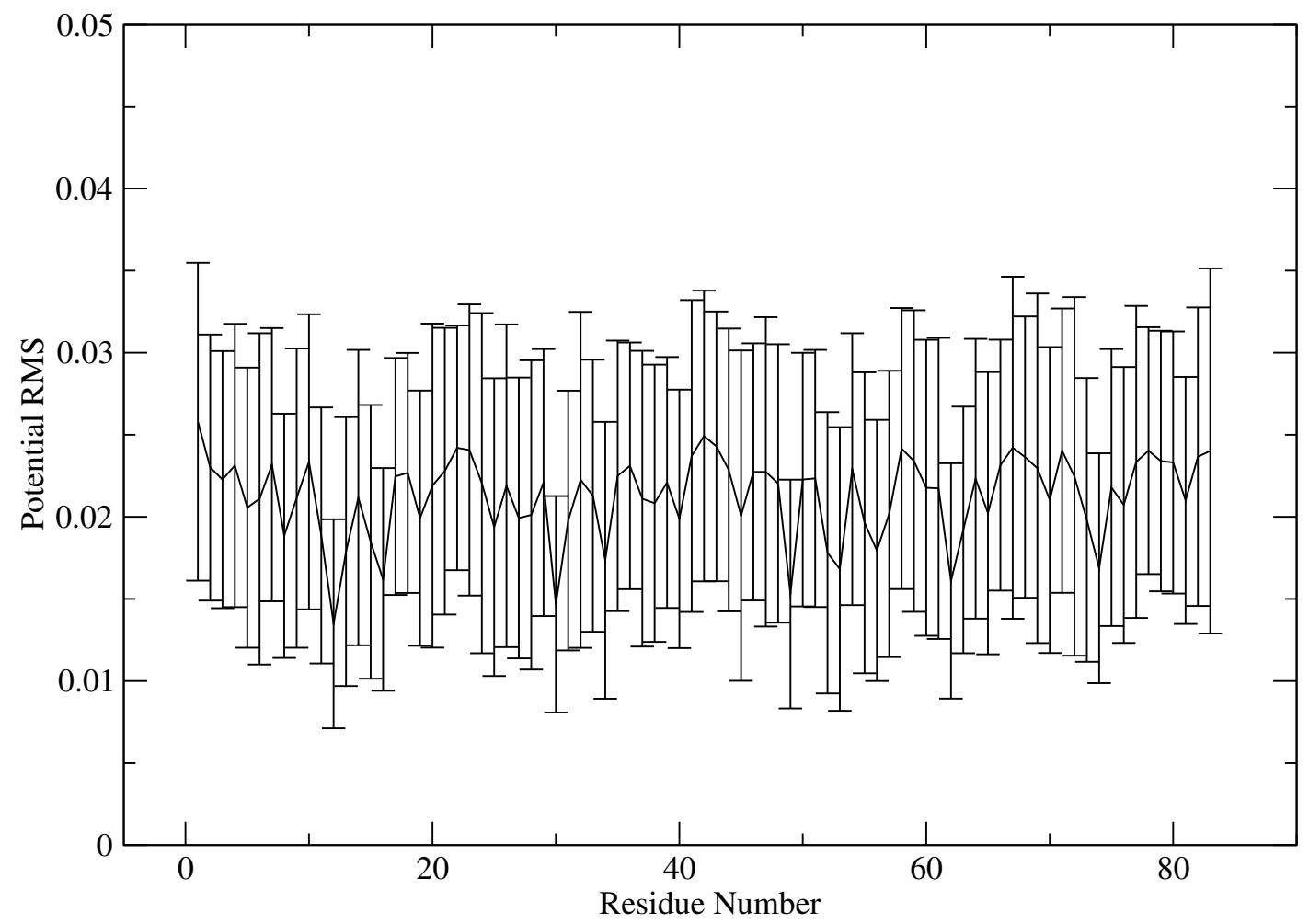

Figure 4.1: RMS of electrostatic potential per residue in equilibrium fixed atomic charge MD simulation. A short peptide (PDB id: 2JOF) is used here.

In order to determine what gaussian distribution that the RMS obeys and to determine the threshold value, several conventional MD simulations with AMBER fixed charge force field are conducted. The electrostatic potential is recorded. And potential RMS between consecutive time points is fitted to gaussian distributions. It turns out that the potential RMS is correlated with the electrostatic property of residues. Nonpolar residues, such as valine, leucine and isoleucine, present lower mean value of potential RMS as well as lower standard deviation. This is expected since such residues contribute less to electrostatic interactions than other polar residues. Nonetheless, typical distribution of potential RMS has a mean value of about $0.02 \mathrm{e} / \AA$, as shown in Fig 4.1 .

In Fig 4.1, potential RMS distribution per residue is plotted. Even though the position and width of distributions vary from residue to residue, it is clear that electrostatic potential acting on all residues do present gaussian distribution. 


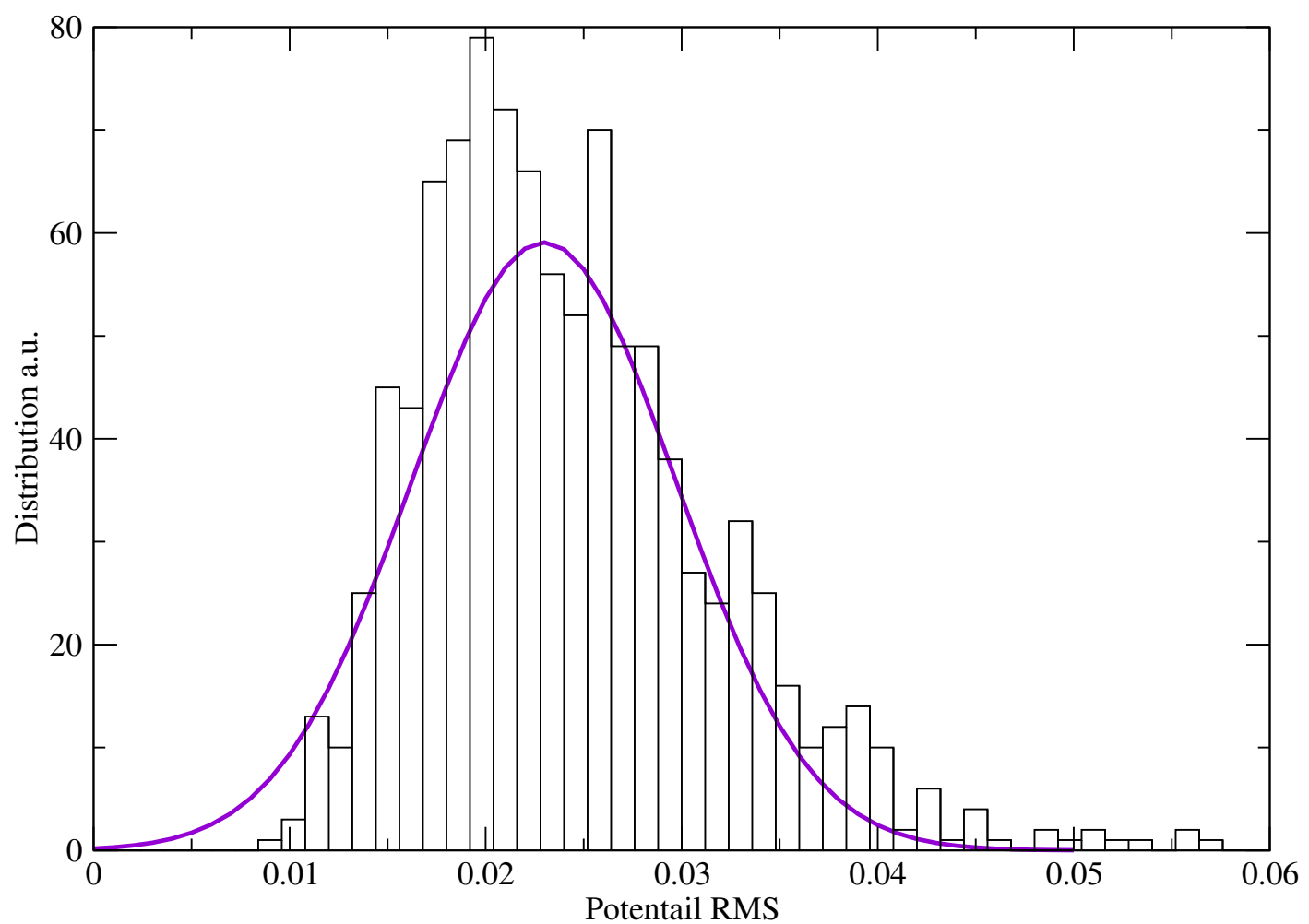

Figure 4.2: Distribution of potential RMS fitted to Gaussian distribution. 
As shown in Fig 4.2, a typical potential RMS distribution can be fitted to Gaussian form, with mean value at $\sim 0.023$. The fitted gaussian in Fig 4.2 is as follow.

$$
y=\frac{1}{6.8 \times 10^{-3} \sqrt{2 \pi}} \exp \left(-\left(\frac{x-0.023}{1.36 \times 10^{-2}}\right)^{2}\right)
$$

Here I chose a single value threshold to initiate charge updating. This means lower probability of charge updating for those residues that exhibit lower potential RMS. As mentioned above, nonpolar residues exhibit smaller potential RMS. This is so because they contribute less to electrostatic energy than polar residues. On the other hand, the polarizability of nonpolar residues is lower than polar residues, hence atomic charge of these residues changes mildly under polarization. So single threshold value for all residue is feasible.

Now, the threshold value shall be outside the gaussian distribution of potential RMS. Here the value of $0.05 \mathrm{e} / \AA$ is used, which correspond to about $\mu+4 \sigma$ of the RMS distribution. Since the basic idea is to make PPC responsive to local environmental change, this new PPC-update scheme is named environment response polarized protein specific charge (ERPPC).

\subsubsection{Implementation}

The implementation is straightforward in this case. The potential RMS per residue is checked every constant time interval. As discussed above, length of this interval depends on the system we're studying. It should be as least long enough to allow reaching equilibrium after each charge update. It is easy to determine by running short MD simulations. If the potential RMS between current time and previous time point is greater than the threshold value, the corresponding residue is subjected to charge updating. The charge updating procedure is standard. Details can be found elsewhere. ${ }^{47}$ Briefly speaking, the residues whose charge is to be fitted are subjected to DFT calculation with caps. All other charged points in the system are treated as background during DFT calculation. The atomic charge is 
fitted with RESP method with a more numerically stable algorithm. $\stackrel{48}{\text { Iteration }}$ is conducted if necessary to preserve self-consistency. At last, the newly fitted charge is past to MD parameter set to continue the simulation. To preserve consistency between newly fitted charge and other force field parameters, the level of quantum chemistry calculation should be same as used in deriving atomic charge for the force field. Here we are working with AMBER03 parameter set, hence B3LYP/6-31G* level of theory is used. All implementation is accomplished with the SANDER module of AMBER10. ${ }^{96}$ DFT calculations are done with Gaussian 09 software package.

\subsubsection{Molecular Dynamics Protocol}

The newly proposed environment response polarized protein specific charge is utilized to study the WpD loop dynamics of Yersinia protein tyrosine phosphatase (YopH). The initial structure of YopH is taken from the Protein Data Bank (PDB id: 1 YPT) ${ }^{117}$ Despite the available structure of YopH with open and closed WpD loop, the open $\mathrm{WpD}$ loop structure is used in this case, since the open and closed state are separated by minimal energy barrier.120 The structure is prepared with LEAP module of AMBER10 package, applying AMBER03 parameter set. First, the protonation states of residues are set according to the charge states at physiological conditions. Then the protein molecule is solvated with a octahedron TIP3P $\mathrm{P}^{64}$ box. All protein atoms are at least $12 \AA$ from box side. The solvated protein is neutralized with counterions before subjected to a thorough minimization. The solvent molecules and counterions are first minimized with all protein atoms restrained. Then the whole system is minimized with first steepest descent then conjugate gradient to reach convergent energy.

The MD simulation is initiated with random speed generated according to Maxwell-Boltzmann distribution at $10 \mathrm{~K}$. Then the system is heated slowly to reach temperature of $310 \mathrm{~K}$, and is stabilized for at least $100 \mathrm{ps}$. The equilibration process is divided into two phases. First, the whole system is equilibrated at 310 $\mathrm{K}$ with constant volume (NVT) for $100 \mathrm{ps}$. Then, pressure coupling is applied to 
keep the pressure constant at 1 bar (NPT) for 500 ps. At last, the production simulation is performed under constant temperature $(310 \mathrm{~K})$ and constant pressure (1 bar). Periodic boundary conditions are applied at all time. Nonbonded cutoff is set to $12 \AA$ for all simulations. Berendsen barostat ${ }^{123}$ is used to maintain constant pressure isotropically. Langevin dynamics is used to obtain constant temperature with collision frequency of $4 \mathrm{ps}^{-1}$. The electrostatic interactions are calculated with particle mesh Ewald (PME) method. ${ }^{[1 / 72}$ Position restraint is applied to protein atoms during heating and NVT equilibration. All bonds involves hydrogen are constrained with SHAKE algorithm. ${ }^{97}$ The time step for all simulations is $2 \mathrm{fs}$. All MD simulations are conducted with the SANDER module of AMBER10 package. ${ }^{96}$ AmberTools software package, especially CPPTRAJ, $\stackrel{124}{15}$ ised to analyze trajectories. 


\subsection{Results And Discussion}

\subsubsection{Distinguishing Open And Closed States}

The definition of open and closed states of YopH binding pocket is critical to our simulation study. Due to the insufficiency of force field model and the limitation of simulated time scale, defining open and closed states based on the $\chi^{2,1}$ dihedral of TRP354 (dihedral CA-CB-CG-CD1), as in the experimental study, ${ }^{120}$ would be problematic. With an all-atom simulation, the size of opening to the binding pocket can be directly observed. Therefore, defining open and closed states of YopH binding pocket by measuring the size of the pocket opening is intuitive and straightforward. Similar measure has been taken by $\mathrm{Hu}$ et.al ${ }^{122}$ in their study of YopH. However, the distance values of pocket opening, adopted by $\mathrm{Hu}$ et.al., 122 to distinguish open and closed states are straight from crystal structures. This makes no sense because the distance values in the crystal structure can be seen as the average values of open and closed states. Using these values as the boundaries of the open and closed states is inappropriate.

The unbound YopH molecules that reside in binding pocket open and closed states are equally populated. There is a small energy barrier between open and closed states. Hence, there should be sampling both in open and closed states during a sufficient long (several times of the $\mathrm{WpD}$ loop motion period of $\sim 4 \mathrm{~ns}$ ) MD simulation. Here, the pocket opening size to determine whether the $\mathrm{WpD}$ loop is in open or closed state, is extracted from MD simulation coupled with principal component analysis (PCA).

GLN357 is at the third position of WpD loop (355-358). The positional movement of its backbone atoms is essential for $\mathrm{WpD}$ loop to complete the transition from open to close. On the other hand, VAL407 resides on the bottom of the binding pocket. So, the distance between C $\alpha$ atoms of GLN357 and VAL407 is characterized to represent the size of binding pocket opening. One of our simulation trajectories of $20 \mathrm{~ns}$ in length is subjected to principal component analysis. The result of PCA shows that the most significant mode of motion corresponds to 


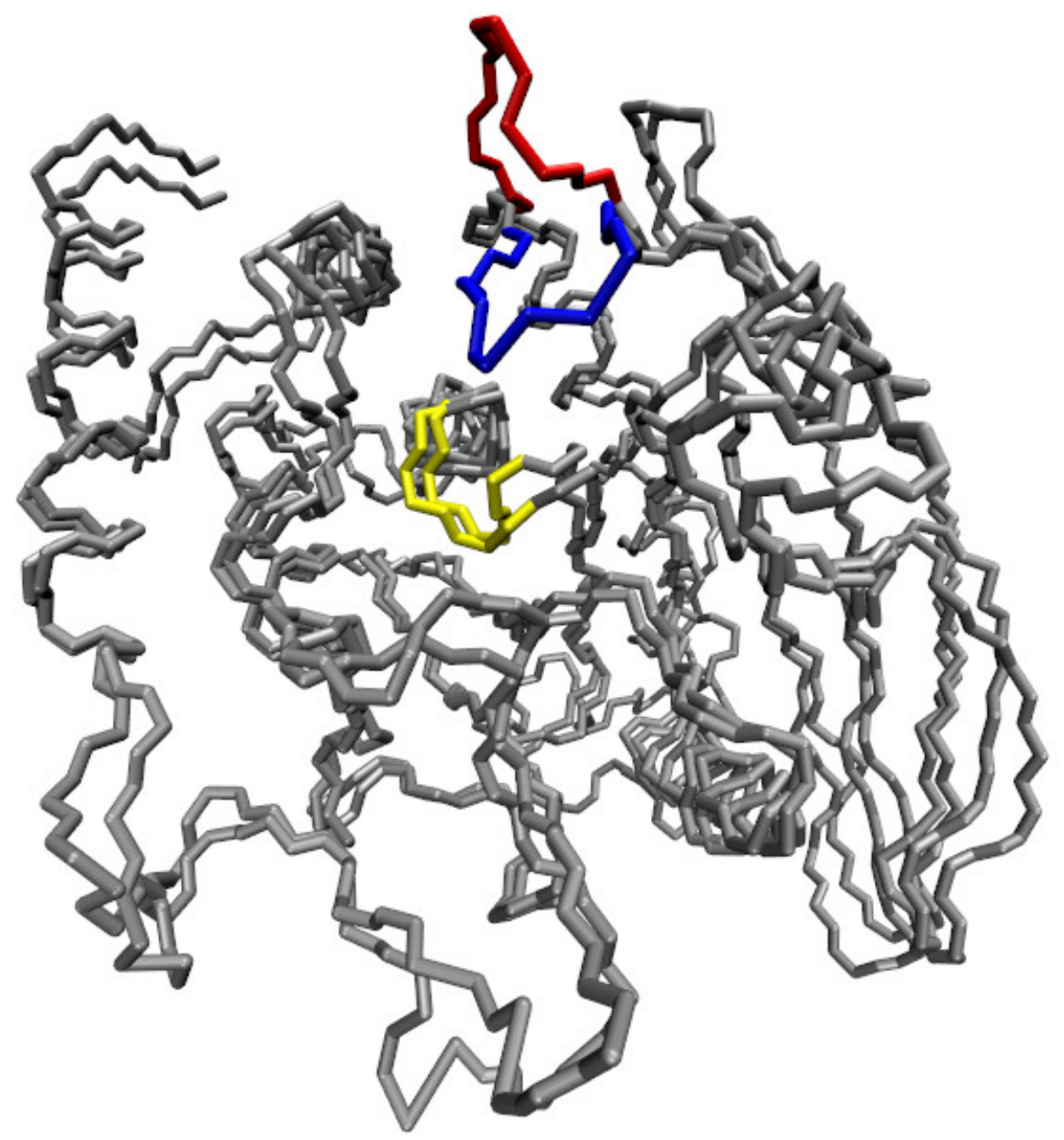

Figure 4.3: Overlap of open and closed backbone structures of YopH determined with PCA. Red: WpD loop in open state. Blue: WpD loop in closed state. Yellow: P-loop. Gray: YopH backbone.

the fluctuation of loops L1 and L6, other than the WpD loop. So, the first 8 eigenvectors are used to acquire the open state with the largest $D_{C \alpha 357-407}$ of $19.88 \AA$ and the closed state with the smallest distance value of $8.69 \AA$. The corresponding structure is drawn in Figure 4.3 .

The mean value of $14.285 \AA$ is set to distinguish the open and closed states. It is reported that the position displacement of GLN357 $\mathrm{C} \alpha$ atom can be as much as $7 \AA^{125}$ ). Taking $14.285 \AA$ as the boundary value infers that the averaged distances 
between GLN357 and VAL407 C $\alpha$ atoms are 10.785 and $17.785 \AA$, for closed and open states respectively. These values correspond very well with crystal structures of YopH.

\subsubsection{Dynamics of the $\mathrm{WpD}$ loop}

The MD simulations of YopH are performed in explicit water starting with $\mathrm{WpD}$ loop in open conformation (PDB id 1YPT). Simulations either with AMBER03 fixed charge or with ERPPC are conducted. With each simulation methods, two $20 \mathrm{~ns}$ long trajectories are obtained with different initial velocity to achieve more extensive sampling. Potential energy and backbone root mean square deviation (RMSD) are inspected to ensure all simulations had reached equilibrium.

First of all, B-factors, which characterize the atomic positional fluctuation, of YopH backbone atoms are calculated with all snapshots in equilibrium. The B-factor is calculated with

$$
B_{i}=\frac{8 \pi^{2}}{3}<\Delta r_{i}^{2}>
$$

B-factor data from AMBER and ERPPC simulations as well as the crystal structure are plotted in Figure 4.4. All loops can be clearly identified from both simulations with AMBER fixed charge or ERPPC charge. As shown in Figure 4.4, the B-factors calculated from ERPPC simulation corresponds better with crystal B-factor data. In AMBER simulations, increased B-factors are observed at loops surrounding ( $\mathrm{WpD}$ loop and L2) or far away (L4 and L5) from the binding pocket. It is very clear that in both simulations, the atomic fluctuation of loop 1 backbone is more significant than in its crystal structure. This may due to the environmental difference, as in crystal, the protein molecules are packed together tightly.

The backbone of WpD loop exhibits more fluctuation in AMBER03 simulation than ERPPC simulation. It indicates that in AMBER03 simulation, the WpD loop presents more positional displacement. Through further investigation, it turns out that in AMBER03 simulation, $\mathrm{WpD}$ loop remains in open state most of the time. We'll look into more details later. 


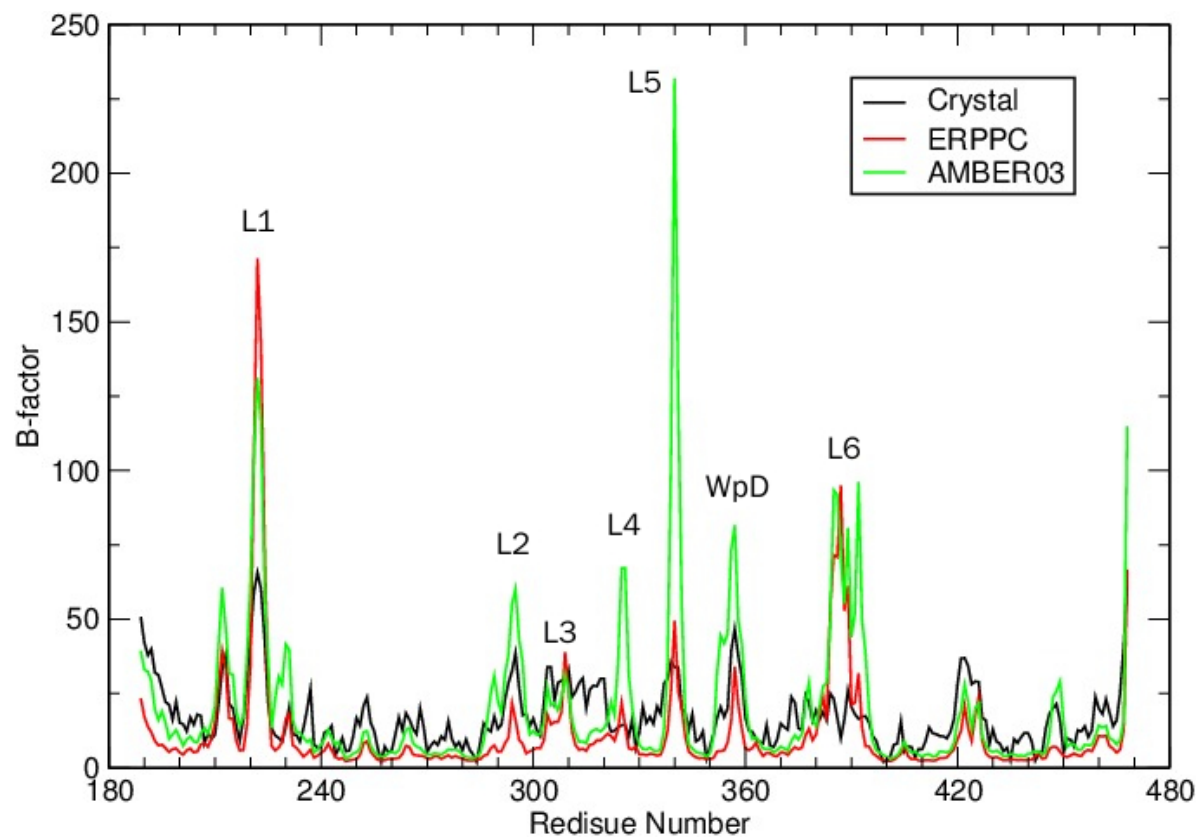

Figure 4.4: Backbone B-factors of YopH per residue. Data from simulations with AMBER and ERPPC, as well as from crystal structure, are plotted. B-factor values from simulation are computed according to equation 4.4. ERPPC data corresponds better with crystal data than AMBER. 


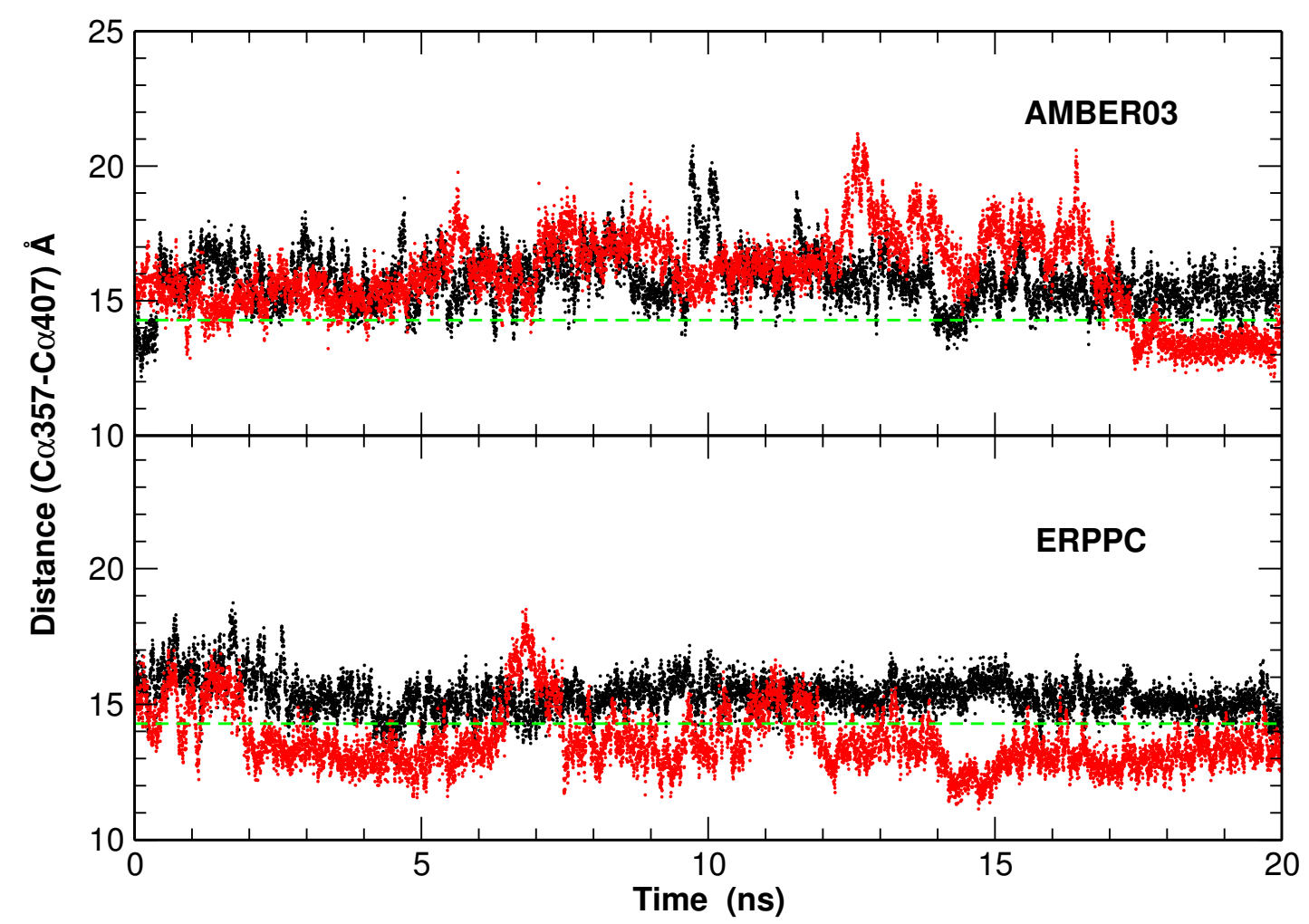

Figure 4.5: $\mathrm{C} \alpha 357-\mathrm{C} \alpha 407$ distance as a function of time. The distance data are extracted from two $20 \mathrm{~ns}$ long trajectories (black and red). The criterion that distinguishes open and closed state of WpD loop is drawn as green dashed lines.

The distance between C $\alpha$ atoms of GLN357 and VAL407 is examined in the next step. As mentioned before, this distance characterizes the opening size of the binding pocket of YopH. The state that the WpD loop is in can be determined directly with this parameter. The $\mathrm{C} \alpha 357-\mathrm{C} \alpha 407$ distance is plotted in Figure 4.5, as a function of time. Two sets of data from two trajectories are plotted for each simulation model we used, i.e. AMBER03 and ERPPC. The criterion that distinguishes open and closed states is plotted as green dashed lines in Figure 4.5.

All our simulations start with the $\mathrm{WpD}$ open conformation structure. In AMBER03 simulations, both trajectories stay in the $\mathrm{WpD}$ open conformation for most of the simulation time. It only reaches closed state at the beginning half nanosecond, then stays in the open state for the rest of the simulation, in trajectory No.1 (rendered black in Figure 4.5). In trajectory No.2, which is rendered 
red in the upper figure of Figure 4.5. YopH stays in $\mathrm{WpD}$ open state for $\sim 17$ ns before switching to closed state for a very short time of $\sim 3$ ns. Then at the end, the YopH molecule tends to switch back to open state. On the other hand, in simulations with ERPPC, the first trajectory (black in Figure 4.5) exhibits very similar conformation to closed state, though never reaches closed state for a period long enough. The second trajectory (red in the lower figure of Figure 4.5) with ERPPC displays several clear transitions between open and closed states of $\mathrm{WpD}$ loop. The $\mathrm{WpD}$ molecule stays stable in the $\mathrm{WpD}$ closed conformation from $\sim 2$ ns to $\sim 6 \mathrm{~ns}$, and from $\sim 14$ ns onward. From $\sim 1$ ns to $\sim 2 \mathrm{~ns}$, and from $\sim 6.5 \mathrm{~ns}$ to $\sim 7.5 \mathrm{~ns}$ is the open state. This trajectory exhibits more open-close transition than any other simulation trajectories, which implies the advantage ERPPC brings to model loop dynamics. Synthesizing all simulations together, we have the resident time ratio of open over closed states of 1.4 with ERPPC, and 9.6 with AMBER.

It is noteworthy that limited by the resources on hand, we have achieved only two $20 \mathrm{~ns}$ trajectories in each case. The sampling is apparently not extensive enough. However, in this limited length of MD simulation, it is encouraging to see the advantage that ERPPC presents over fixed charge force field in modeling loop dynamics. As discussed before, the protein specific charge which is adapted constantly to the environment is indeed better in describing dynamic behaviors of protein molecule, such as loop dynamics in our case.

\subsubsection{The Importance of CYS403}

The CYS403 located on the P-loop is pivotal to the catalytic activity of YopH. As described in the catalytic process, CYS403 serves as a nucleophile to accept the phosphoryl group dissociated from substrate. To maintain proper enzyme function, the side chain thiol of CYS403 must be deprotonated, as required by its nucleophilic role in the catalytic process. Site-directed mutagenesis studies show that the mutant C403S of YopH is catalytically inactive although it's capable of binding substrates. Further study reveals that the reason of abolishment of reactivity in C403S mutant could be two fold. For one, decreased ncleophilic reactivity 


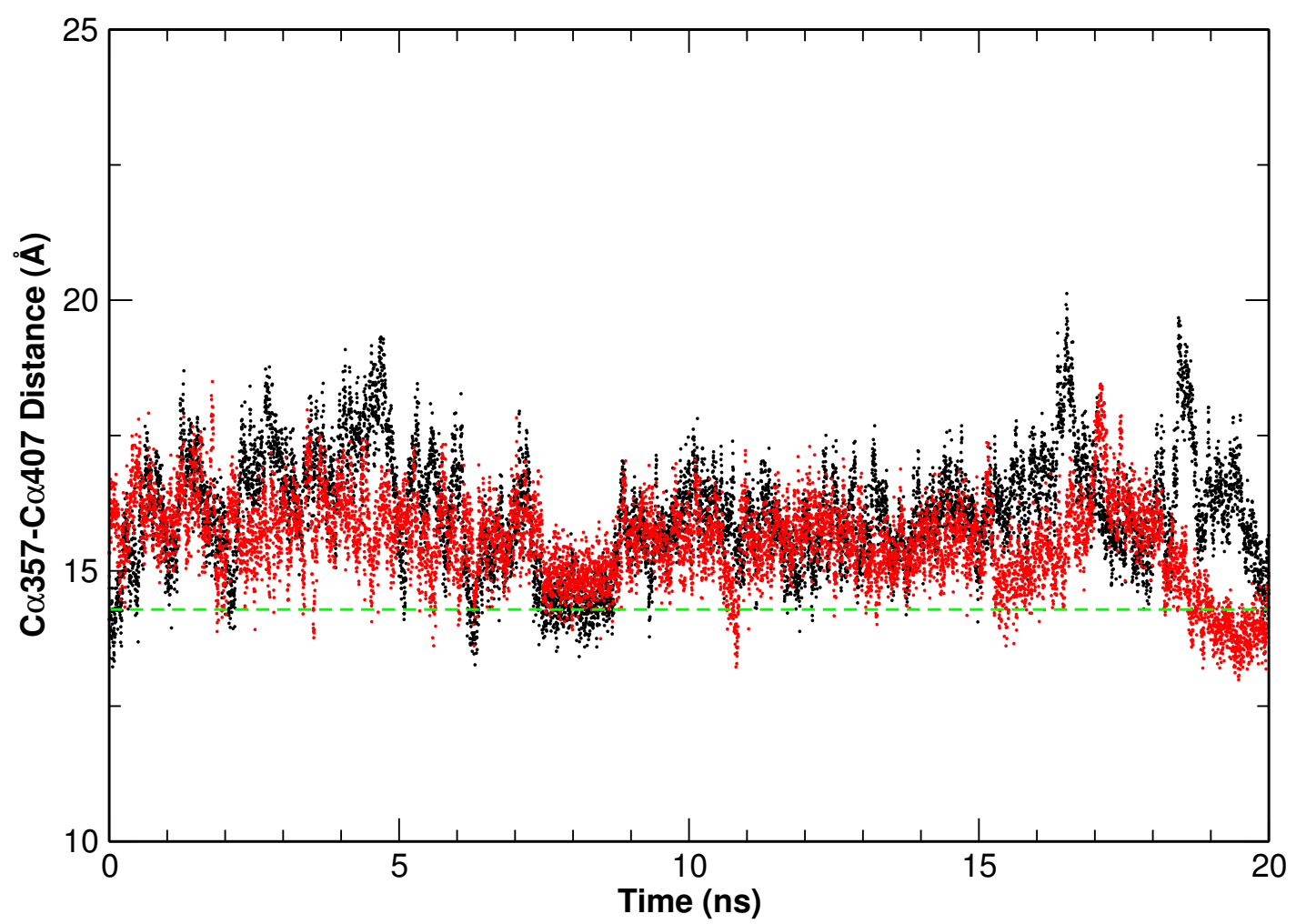

Figure 4.6: $\mathrm{C} \alpha 357-\mathrm{C} \alpha 407$ distance as a function of time in simulations with protonated C403. The color scheme is the same as Figure 4.5.

is induced by the mutation from cysteine to serine. Secondly, there are structural perturbations involved. Here, through the means of MD simulation, the influence on $\mathrm{WpD}$ loop dynamics introduced by $\mathrm{C} 403$ protonation is presented, which furthers our understanding of the role of cysteine 403 in retaining the dynamics of WpD loop.

To illustrate the critical role of cysteine 403 in $\mathrm{WpD}$ dynamics, simulations are conducted with C403 thiol group in protonated state. Two 20 ns trajectories are obtained with ERPPC model. The C 2357 - C $\alpha 407$ distance is plotted in Figure 4.6 .

The YopH molecule is staying at the $\mathrm{WpD}$ open conformation most of the time in both simulations. The only stable closed conformation achieved is at the last 1 ns of the second trajectory. So it's clear that the protonation state of cysteine 403 has influence on the dynamics of $\mathrm{WpD}$ loop. 
Table 4.1: Time consumption of simulations with AMBER and ERPPC. All trajectories have the same 20 ns length.

\begin{tabular}{cccc}
\hline \hline Trajectory Number & Time (hr) & $\begin{array}{c}\text { Percentage of Time } \\
\text { on Charge Updating }\end{array}$ & $\begin{array}{c}\text { Time Consumption } \\
\text { over Conventional MD }\end{array}$ \\
\hline AMBER 1 & 272.48 & & \\
AMBER 2 & 268.60 & & $2.52 \times$ \\
ERPPC 1 & 545.68 & 60.3 & $2.56 \times$ \\
ERPPC 2 & 550.19 & 60.9 & $2.31 \times$ \\
ERPPC 3 & 594.16 & 56.8 & $2.32 \times$ \\
ERPPC 4 & 598.28 & 56.9 & \\
\hline
\end{tabular}

\subsubsection{Computation Performance}

Here we present the timing information on simulations with ERPPC scheme. All simulations conducted in this chapter have been done on the HPC cluster in Nanyang Technological University (NTU). Two computation nodes equipped with two Quad-core cpus each (16 cores in total) are used to run a single simulation. The computing time consumed are presented in Table 4.1.

As can be seen in Table 4.1, the time consumed by an ERPPC simulation is typically 2-3 times comparing to normal MD, depending on the size of simulated system. In our case, ERPPC simulations number 1 and 2 have the same number of atoms. They also have consumed similar amount of computing time. It is similar in the case of ERPPC trajectories 3 and 4. Significantly less time is used comparing to other methods simulating the polarization effect, such as polarizable force field. 


\subsection{Conclusion}

In this chapter, ERPPC charge updating scheme is proposed and applied to study the loop dynamics of YopH. The WpD loop dynamics, which is rather rapid, is critical to the catalytical activity, as well as biological function of YopH. Modeling $\mathrm{WpD}$ loop dynamics correctly is important to further computational study of YopH and other PTPase.

With limited computing resources, several trajectories are obtained for both AMBER and ERPPC model. Though the sampling could be more extensive, reasonable results are analyzed and discussed. By comparing these results, it is found that $\mathrm{WpD}$ loop open-close state transition is observed with simulations governed by ERPPC. However, in simulations with AMBER fixed charge, no meaningful open-close transition is observed. Since this transition is a rapid process and has minimal energy barrier, the WpD loop dynamics is better represented with ERPPC scheme, rather than AMBER fixed charge. It is also found out that the protonation state of CYS403 has influence on WpD loop dynamics. With CYS403 side chain thiol group in its protonated state, the WpD loop didn't reach closed state within our simulation length. This result supports the theory that the C403S mutation of YopH not only removes a nucleophilic group, but also alters the dynamics of WpD loop which governs the opening of substrate binding pocket. Furthermore, simulations with ERPPC scheme consume $\sim 2-3$ times computation time comparing to MD simulations with fixed charge. It is less than other polarizable methods.

Correctly modeling loop dynamics requires more accurate energy functions than current force fields. Through this simulation, ERPPC scheme exhibits potentials of correctly modeling protein dynamics. In order to fully exploit the capability of ERPPC, more study is to be done. Besides, it is possible to improve ERPPC over the study presented here, since the information contained in electrostatic potential is not fully utilized currently. I'm very optimistic on further development and application of ERPPC scheme. 


\section{Chapter 5}

\section{Concluding Remarks and Future Perspectives}

In this thesis, molecular dynamics simulations with different levels of polarization are conducted upon several intriguing questions, including biomolecule-graphene interaction, peptides structural stability and enzyme substrate binding pocket regulation.

The first study is about protein and DNA interacting with pristine graphene, which is a star material with huge application potential. The simulations are done with simple conventional force field model. Interesting results are obtained by performing the simulation study. It shows that even though graphene is a $2-\mathrm{D}$ thin layer of carbon atoms, it exhibits properties similar to solid. The adsorption onto graphene surface could utterly destroy the structure of some protein. Proteins that fold tightly might survive the adsorption process. On the other hand, double helix DNA molecules prefer "standing up" position when inserted into graphene layers. Though insights are revealed by the simulations, properties that are more important to graphene application, such as quenching and electronic dispersion, can not be explored with our model. More detailed representation is necessary to study these properties.

The next study introduces a little polarization into simulations of protein solution simulation. Two interesting, yet excessively simple, models of backbone fluctuating charge are explored and compared. It turns out these two models 
both exhibit stronger backbone interaction than AMBER fixed charge. However, the overall performances of both model on protein molecules are not satisfactory, especially semifluctuating charge model.

Last, the PPC-update scheme is improved with environmental electrostatic interaction information, i.e. electrostatic potential. It is utilized to determine whether to perform PPC-update on residues during MD simulation, hence named environment response polarized protein specific charge (ERPPC). The new scheme improves both accuracy and efficiency of PPC-update simulation. A case study on the loop dynamics of YopH shows that ERPPC reproduced the open-close transition of YopH WpD loop within our simulation time, while AMBER force field failed. The study shows the potential of ERPPC scheme to perform molecular dynamics simulation with higher accuracy than conventional force field, and lower computational cost than polarizable force field.

Incorporating polarization into molecular dynamics simulation is both challenging and attractive. ERPPC scheme presents potentials on this perspective. The new ERPPC scheme can be improved in several ways. First, the efficiency and/or accuracy of PPC charge fitting can be improved. With help from new calculation platform, such as GPU computing, new method is to be developed to produce better PPC. Second, the updating procedure and updating criterion in ERPPC should be optimized and improved. Simple measure like the root mean square of potential which is used in this thesis is limited in capability. To sum up, works can be done to improve all-atom molecular dynamics simulation. Both accuracy and efficiency of MD simulation are to be advanced in the future. 


\section{List of Publications}

(i) Sun, Tiedong; Wei, Caiyi ; Neo, Ni Wei Charlie and Zhang, Dawei "Misfolding of a polyalanine variant due to lack of electrostatic polarization effects" Theoretical Chemistry Accounts 132,5,1354

(ii) Sun, Tiedong; Chan, Kwok Kiong; Su, Haibin and Zhang, Dawei "Dissimilar stability of proteins in graphene bilayer: a molecular dynamics study" Molecular Physics 111,4,545

(iii) Chan, Kwok Kiong ; Sun, Tiedong ; Lu, Yunpeng ; Shao, Fangwei and Zhang, Dawei "Self-assembly of DNA duplex in graphene bilayer" Molecular Physics 111,8,1053

(iv) Xu, Zhijun ; Lazim, Raudah ; Sun, Tiedong; Mei, Ye and Zhang, Dawei "Solvent effect on the folding dynamics and structure of E6-associated protein characterized from ab initio protein folding simulations" The Journal of Chemical Physics 136,13,135102

(v) Lazim, Raudah ; Wei, Caiyi ; Sun, Tiedong and Zhang, Dawei "Ab initio folding of extended -helix: A theoretical study about the role of electrostatic polarization in the folding of helical structures" Proteins: Structure, Function, and Bioinformatics 81,9,1610 


\section{References}

[1] Shaw, D. E.; Maragakis, P.; Lindorff-Larsen, K.; Piana, S.; Dror, R. O.; Eastwood, M. P.; Bank, J. A.; Jumper, J. M.; Salmon, J. K.; Shan, Y.; Wriggers, W. Science 2010, 330, 341-346.

[2] Shaw, D. E. et al. Millisecond-scale Molecular Dynamics Simulations on Anton. 2009; http://doi.acm.org/10.1145/1654059.1654099.

[3] Tozzini, V. Current Opinion in Structural Biology 2005, 15, 144 - 150, Theory and simulation/Macromolecular assemblages.

[4] Nielsen, S. O.; Lopez, C. F.; Srinivas, G.; Klein, M. L. Journal of Physics: Condensed Matter 2004, 16, R481.

[5] Shirvanyants, D.; Ding, F.; Tsao, D.; Ramachandran, S.; Dokholyan, N. V. The Journal of Physical Chemistry B 2012, 116, 8375-8382.

[6] Emperador, A.; Solernou, A.; Sfriso, P.; Pons, C.; Gelpi, J. L.; FernandezRecio, J.; Orozco, M. Journal of Chemical Theory and Computation 2013, 9, 12221229 .

[7] Iftimie, R.; Minary, P.; Tuckerman, M. E. Proceedings of the National Academy of Sciences of the United States of America 2005, 102, 6654-6659.

[8] Liu, L.-M.; Laio, A.; Michaelides, A. Phys. Chem. Chem. Phys. 2011, 13, 13162-13166. 
[9] Liu, H.; Elstner, M.; Kaxiras, E.; Frauenheim, T.; Hermans, J.; Yang, W. Proteins: Structure, Function, and Bioinformatics 2001, 44, 484-489.

[10] Liang, W.; Chapman, C. T.; Li, X. The Journal of Chemical Physics 2011, 134, 184102.

[11] Alder, B. J.; Wainwright, T. E. The Journal of Chemical Physics 1959, 31, 459-466.

[12] Rahman, A. Phys. Rev. 1964, 136, A405-A411.

[13] Sugita, Y.; Okamoto, Y. Chemical Physics Letters 1999, 314, 141 - 151.

[14] Okamoto, Y. Journal of Molecular Graphics and Modelling 2004, 22, 425 439, Conformational Sampling.

[15] Patel, J. S.; Berteotti, A.; Ronsisvalle, S.; Rocchia, W.; Cavalli, A. Journal of Chemical Information and Modeling 2014, 54, 470-480.

[16] Verlet, L. Phys. Rev. 1967, 159, 98-103.

[17] Swope, W. C.; Andersen, H. C.; Berens, P. H.; Wilson, K. R. The Journal of Chemical Physics 1982, 76, 637-649.

[18] Cornell, W. D.; Cieplak, P.; Bayly, C. I.; Gould, I. R.; Merz, K. M.; Ferguson, D. M.; Spellmeyer, D. C.; Fox, T.; Caldwell, J. W.; Kollman, P. A. Journal of the American Chemical Society 1995, 117, 5179-5197.

[19] Duan, Y.; Wu, C.; Chowdhury, S.; Lee, M. C.; Xiong, G.; Zhang, W.; Yang, R.; Cieplak, P.; Luo, R.; Lee, T.; Caldwell, J.; Wang, J.; Kollman, P. Journal of Computational Chemistry 2003, 24, 1999-2012.

[20] Brooks, B. R.; Bruccoleri, R. E.; Olafson, B. D.; States, D. J.; Swaminathan, S.; Karplus, M. Journal of Computational Chemistry 1983, 4, 187217. 
[21] Jorgensen, W. L.; Tirado-Rives, J. Journal of the American Chemical Society 1988, 110, 1657-1666.

[22] Schmid, N.; Eichenberger, A.; Choutko, A.; Riniker, S.; Winger, M.; Mark, A.; van Gunsteren, W. European Biophysics Journal 2011, 40, 843856.

[23] Lindorff-Larsen, K.; Piana, S.; Palmo, K.; Maragakis, P.; Klepeis, J. L.; Dror, R. O.; Shaw, D. E. Proteins: Structure, Function, and Bioinformatics 2010, 78, 1950-1958.

[24] Li, D.-W.; Brschweiler, R. Angewandte Chemie International Edition 2010, 49, 6778-6780.

[25] Sprik, M. The Journal of Physical Chemistry 1991, 95, 2283-2291.

[26] Li, Y. L.; Mei, Y.; Zhang, D. W.; Xie, D. Q.; Zhang, J. Z. H. J Phys Chem B 2011, 115, 10154-10162.

[27] Gao, Y.; Lu, X.; Duan, L. L.; Zhang, J. Z. H.; Mei, Y. The Journal of Physical Chemistry B 2012, 116, 549-554.

[28] Riniker, S.; van Gunsteren, W. F. The Journal of Chemical Physics 2011, $134,-$

[29] Lamoureux, G.; Roux, B. The Journal of Chemical Physics 2003, 119, 30253039.

[30] Ponder, J. W.; Wu, C.; Ren, P.; Pande, V. S.; Chodera, J. D.; Schnieders, M. J.; Haque, I.; Mobley, D. L.; Lambrecht, D. S.; DiStasio, R. A.; Head-Gordon, M.; Clark, G. N. I.; Johnson, M. E.; HeadGordon, T. The Journal of Physical Chemistry B 2010, 114, 2549-2564, PMID: 20136072. 
[31] Yu, H.; van Gunsteren, W. F. Computer Physics Communications 2005, $172,69-85$.

[32] Morita, A.; Kato, S. Journal of the American Chemical Society 1997, 119, 4021-4032.

[33] Rick, S. W.; Stuart, S. J.; Berne, B. J. The Journal of Chemical Physics 1994, 101, 6141-6156.

[34] Zhao, D.-X.; Yu, L.; Gong, L.-D.; Liu, C.; Yang, Z.-Z. The Journal of Chemical Physics 2011, 134, 194115.

[35] Patel, S.; Brooks, C. L. Journal of Computational Chemistry 2004, 25, 1-16.

[36] Piquemal, J.-P.; Cisneros, G. A.; Reinhardt, P.; Gresh, N.; Darden, T. A. The Journal of Chemical Physics 2006, 124, 104101.

[37] Morita, A.; Kato, S. The Journal of Chemical Physics 1998, 108, 6809-6818.

[38] Manthe, U.; Kppel, H. The Journal of Chemical Physics 1990, 93, 345-356.

[39] Goedecker, S. Rev. Mod. Phys. 1999, 71, 1085-1123.

[40] Junquera, J.; Paz, O.; Sánchez-Portal, D.; Artacho, E. Phys. Rev. B 2001, $64,235111$.

[41] Zhang, D. W.; Zhang, J. Z. H. Journal of Chemical Physics 2003, 119, 3599-3605.

[42] Zhang, D. W.; Chen, X. H.; Zhang, J. Z. H. Journal of Computational Chemistry 2003, 24, 1846-1852.

[43] Gordon, M. S.; Fedorov, D. G.; Pruitt, S. R.; Slipchenko, L. V. Chemical Reviews 2012, 112, 632.

[44] Gao, A. M.; Zhang, D. W.; Zhang, J. Z.; Zhang, Y. Chemical Physics Letters 2004, 394, $293-297$. 
[45] Xiang, Y.; Zhang, D. W.; Zhang, J. Z. H. Journal of Computational Chemistry 2004, 25, 1431-1437.

[46] Zhang, D. W.; Xiang, Y.; Gao, A. M.; Zhang, J. Z. H. The Journal of Chemical Physics 2004, 120, 1145-1148.

[47] Ji, C. G.; Mei, Y.; Zhang, J. Z. H. BIOPHYSICAL JOURNAL 2008, 95, 1080-1088.

[48] Zeng, J.; Duan, L.; Zhang, J. Z.; Mei, Y. Journal of Computational Chemistry 2012, 847-853.

[49] Bayly, C. I.; Cieplak, P.; Cornell, W.; Kollman, P. A. The Journal of Physical Chemistry 1993, 97, 10269-10280.

[50] Wang, Y.; Li, Z.; Wang, J.; Li, J.; Lin, Y. Trends in Biotechnology 2011, 29, 205-212, doi: 10.1016/j.tibtech.2011.01.008.

[51] Georgakilas, V.; Otyepka, M.; Bourlinos, A. B.; Chandra, V.; Kim, N.; Kemp, K. C.; Hobza, P.; Zboril, R.; Kim, K. S. Chemical Reviews 2012, 112, 6156-6214.

[52] Lee, C.; Wei, X.; Kysar, J. W.; Hone, J. Science 2008, 321, 385-388.

[53] Balandin, A. A.; Ghosh, S.; Bao, W.; Calizo, I.; Teweldebrhan, D.; Miao, F.; Lau, C. N. Nano Letters 2008, 8, 902-907.

[54] Avouris, P.; Chen, Z.; Perebeinos, V. Nat Nano 2007, 2, 605-615, 10.1038/nnano.2007.300.

[55] Zhang, Y.; Tan, Y.-W.; Stormer, H. L.; Kim, P. Nature 2005, 438, 201-204, $10.1038 /$ nature04235.

[56] Liu, Z.; Robinson, J. T.; Sun, X.; Dai, H. Journal of the American Chemical Society 2008, 130, 10876-10877. 
[57] Mohanty, N.; Berry, V. Nano Letters 2008, 8, 4469-4476.

[58] Zhang, J.; Zhang, F.; Yang, H.; Huang, X.; Liu, H.; Zhang, J.; Guo, S. Langmuir 2010, 26, 6083-6085, PMID: 20297789.

[59] Alwarappan, S.; Liu, C.; Kumar, A.; Li, C.-Z. The Journal of Physical Chemistry C 2010, 114, 12920-12924.

[60] Liu, J.; Fu, S.; Yuan, B.; Li, Y.; Deng, Z. Journal of the American Chemical Society 2010, 132, 7279-7281, PMID: 20462190.

[61] Johansson, M. U.; de Chteau, M.; Wikstrm, M.; Forsn, S.; Drakenberg, T.; Bjrck, L. Journal of Molecular Biology 1997, 266, 859 - 865.

[62] Gronenborn, A.; Filpula, D.; Essig, N.; Achari, A.; Whitlow, M.; Wingfield, P.; Clore, G. Science 1991, 253, 657-661.

[63] Sorin, E. J.; Pande, V. S. Biophysical journal 2005, 88, 2472-2493.

[64] Jorgensen, W. L.; Chandrasekhar, J.; Madura, J. D.; Impey, R. W.; Klein, M. L. The Journal of Chemical Physics 1983, 79, 926-935.

[65] Humphrey, W.; Dalke, A.; Schulten, K. Journal of Molecular Graphics 1996, $14,33-38$.

[66] Frishman, D.; Argos, P. Proteins: Structure, Function, and Bioinformatics 1995, 23, 566-579.

[67] Hess, B. Journal of Chemical Theory and Computation 2008, 4, 116-122.

[68] Bussi, G.; Donadio, D.; Parrinello, M. JOURNAL OF CHEMICAL PHYSICS 2007, 126.

[69] Nos, S.; Klein, M. Molecular Physics 1983, 50, 1055-1076.

[70] Parrinello, M.; Rahman, A. Journal of Applied Physics 1981, 52, 7182-7190, cited By (since 1996) 1377. 
[71] Darden, T.; York, D.; Pedersen, L. The Journal of Chemical Physics 1993, 98, 10089-10092.

[72] Essmann, U.; Perera, L.; Berkowitz, M. L.; Darden, T.; Lee, H.; Pedersen, L. G. The Journal of Chemical Physics 1995, 103, 8577-8593.

[73] Hess, B.; Kutzner, C.; van der Spoel, D.; Lindahl, E. Journal of Chemical Theory and Computation 2008, 4, 435-447.

[74] Mittal, J.; Best, R. B. Proceedings of the National Academy of Sciences 2008, 105, 20233-20238.

[75] Schlesinger, A. P.; Wang, Y.; Tadeo, X.; Millet, O.; Pielak, G. J. Journal of the American Chemical Society 2011, 133, 8082-8085.

[76] Tian, J.; García, A. E. The Journal of Chemical Physics 2011, 134, 225101.

[77] Sorin, E. J.; Pande, V. S. Journal of the American Chemical Society 2006, 128, 6316-6317.

[78] Kabsch, W.; Sander, C. Biopolymers 1983, 22, 2577-2637.

[79] Wang, J.; Wang, W.; Huo, S.; Lee, M.; Kollman, P. A. The Journal of Physical Chemistry B 2001, 105, 5055-5067.

[80] Hu, Y.; Li, F.; Bai, X.; Li, D.; Hua, S.; Wang, K.; Niu, L. Chem. Commun. 2011, 47, 1743-1745.

[81] Lu, C.-H.; Yang, H.-H.; Zhu, C.-L.; Chen, X.; Chen, G.-N. Angewandte Chemie International Edition 2009, 48, 4785-4787.

[82] Jang, H.; Kim, Y.-K.; Kwon, H.-M.; Yeo, W.-S.; Kim, D.-E.; Min, D.-H. Angewandte Chemie International Edition 2010, 49, 5703-5707.

[83] Famulok, M.; Hartig, J. S.; Mayer, G. Chemical Reviews 2007, 107, 37153743 . 
[84] You, M.; Chen, Y.; Peng, L.; Han, D.; Yin, B.; Ye, B.; Tan, W. Chem. Sci. 2011, 2, 1003-1010.

[85] Lee, W. C.; Lim, C. H. Y. X.; Shi, H.; Tang, L. A. L.; Wang, Y.; Lim, C. T.; Loh, K. P. ACS Nano 2011, 5, 7334-7341.

[86] Nayak, T. R.; Andersen, H.; Makam, V. S.; Khaw, C.; Bae, S.; Xu, X.; Ee, P.-L. R.; Ahn, J.-H.; Hong, B. H.; Pastorin, G.; zyilmaz, B. ACS Nano 2011, 5, 4670-4678.

[87] Wells, D. B.; Belkin, M.; Comer, J.; Aksimentiev, A. Nano Letters 2012, 12, 4117-4123.

[88] Lv, W.; Guo, M.; Liang, M.-H.; Jin, F.-M.; Cui, L.; Zhi, L.; Yang, Q.-H. J. Mater. Chem. 2010, 20, 6668-6673.

[89] Patil, A. J.; Vickery, J. L.; Scott, T. B.; Mann, S. Advanced Materials 2009, 21, 3159-3164.

[90] Liu, M.; Zhao, H.; Chen, S.; Yu, H.; Quan, X. Chem. Commun. 2012, 48, $564-566$.

[91] Zhao, X. The Journal of Physical Chemistry C 2011, 115, 6181-6189.

[92] Ji, C. G.; Xiao, X.; Zhang, J. Z. H. Journal of Chemical Theory and Computation 2012, 8, 2157.

[93] Andersen, N. H.; Olsen, K. A.; Fesinmeyer, R. M.; Tan, X.; Hudson, F. M.; Eidenschink, L. A.; Farazi, S. R. Journal of the American Chemical Society 2006, 128, 6101-6110, PMID: 16669679.

[94] Honda, S.; Yamasaki, K.; Sawada, Y.; Morii, H. Structure 2004, 12, $1507-$ 1518, doi: 10.1016/j.str.2004.05.022.

[95] Liu, D.; Yao, H.; Chen, Y.; Feng, Y.; Chen, Y.; Wang, J. Biochem. J. 2005, 392, 47-54. 
[96] Case, D. A.; Cheatham, T. E.; Darden, T.; Gohlke, H.; Luo, R.; Merz, K. M.; Onufriev, A.; Simmerling, C.; Wang, B.; Woods, R. J. Journal of Computational Chemistry 2005, 26, 1668-1688.

[97] Ryckaert, J.-P.; Ciccotti, G.; Berendsen, H. J. Journal of Computational Physics 1977, 23, $327-341$.

[98] Yang, S.; Onuchic, J. N.; Garca, A. E.; Levine, H. Journal of Molecular Biology 2007, 372, $756-763$.

[99] Karplus, M. Journal of the American Chemical Society 1963, 85, 2870-2871.

[100] Prez, C.; Lhr, F.; Rterjans, H.; Schmidt, J. M. Journal of the American Chemical Society 2001, 123, 7081-7093, PMID: 11459487.

[101] Cruz, L.; Rao, J. S.; Teplow, D. B.; Urbanc, B. The Journal of Physical Chemistry B 2012, 0, null.

[102] Xu, Y.; Du, D.; Oyola, R. The Journal of Physical Chemistry B 2011, 115, $15332-15338$.

[103] Gilman-Politi, R.; Harries, D. Journal of Chemical Theory and Computation 2011, 7, 3816-3828.

[104] Huang, W.; Lin, Z.; van Gunsteren, W. F. Journal of Chemical Theory and Computation 2011, \%, 1237-1243.

[105] Hwang, S.; Hilty, C. The Journal of Physical Chemistry B 2011, 115, 1535515361.

[106] Florova, P.; Sklenovsky, P.; Banas, P.; Otyepka, M. Journal of Chemical Theory and Computation 2010, 6, 3569-3579.

[107] Lewandowska, A.; Odziej, S.; Liwo, A.; Scheraga, H. A. Biophysical Chemistry 2010, 151, $1-9$. 
[108] Thukral, L.; Smith, J. C.; Daidone, I. Journal of the American Chemical Society 2009, 131, 18147-18152, PMID: 19919102.

[109] Shao, Q.; Yang, L.; Gao, Y. Q. The Journal of Chemical Physics 2009, 130, 195104.

[110] Araki, M.; Tamura, A. Proteins: Structure, Function, and Bioinformatics 2007, 66, 860-868.

[111] Andersen, N. H.; Olsen, K. A.; Fesinmeyer, R. M.; Tan, X.; Hudson, F. M.; Eidenschink, L. A.; Farazi, S. R. Journal of the American Chemical Society 2006, 128, 6101-6110, PMID: 16669679.

[112] Pastor, M. T.; Lpez de la Paz, M.; Lacroix, E.; Serrano, L.; Prez-Pay, E. Proceedings of the National Academy of Sciences 2002, 99, 614-619.

[113] Isegawa, M.; Kato, S. Journal of Chemical Theory and Computation 2009, 5, 2809-2821.

[114] Xu, Z. J.; Mei, Y.; Duan, L. L.; Zhang, D. W. CHEMICAL PHYSICS LETTERS 2010, 495, 151-154.

[115] Duan, L. L.; Mei, Y.; Zhang, D.; Zhang, Q. G.; Zhang, J. Z. H. Journal of the American Chemical Society 2010, 132, 11159-11164.

[116] Xu, Z.; Lazim, R.; Mei, Y.; Zhang, D. Chemical Physics Letters 2012, 539 $-540,239-244$.

[117] Stuckey, J. A.; Schubert, H. L.; Fauman, E. B.; Zhang, Z.-Y.; Dixon, J. E.; Saper, M. A. Nature 1994, 370, 571-575, 10.1038/370571a0.

[118] Zhang, Z.-Y. Current Opinion in Chemical Biology 2001, 5, 416 - 423.

[119] Zhang, Z.-Y. Progress in Nucleic Acid Research and Molecular Biology 2003, 73, $171-220$. 
[120] Juszczak, L. J.; Zhang, Z.-Y.; Wu, L.; Gottfried, D. S.; Eads, D. D. Biochemistry 1997, 36, 2227-2236.

[121] Peters, G. H.; Frimurer, T. M.; Andersen, J. N.; Olsen, O. H. Biophysical Journal 1999, 77, $505-515$.

[122] Hu, X.; Stebbins, C. E. Biophys J 2006, 91, 948-956.

[123] Berendsen, H. J. C.; Postma, J. P. M.; van Gunsteren, W. F.; DiNola, A.; Haak, J. R. The Journal of Chemical Physics 1984, 81, 3684-3690.

[124] Roe, D. R.; Cheatham, T. E. Journal of Chemical Theory and Computation 2013, 9, 3084-3095.

[125] Schubert, H. L.; Fauman, E. B.; Stuckey, J. A.; Dixon, J. E.; Saper, M. A. Protein Science 1995, 4, 1904-1913. 